\title{
Aorden
}

\section{Place, (In)Equality and Gender}

A Mapping of Challenges and Best Practices in Relation to Gender, Education and Population Flows in Nordic Peripheral Areas

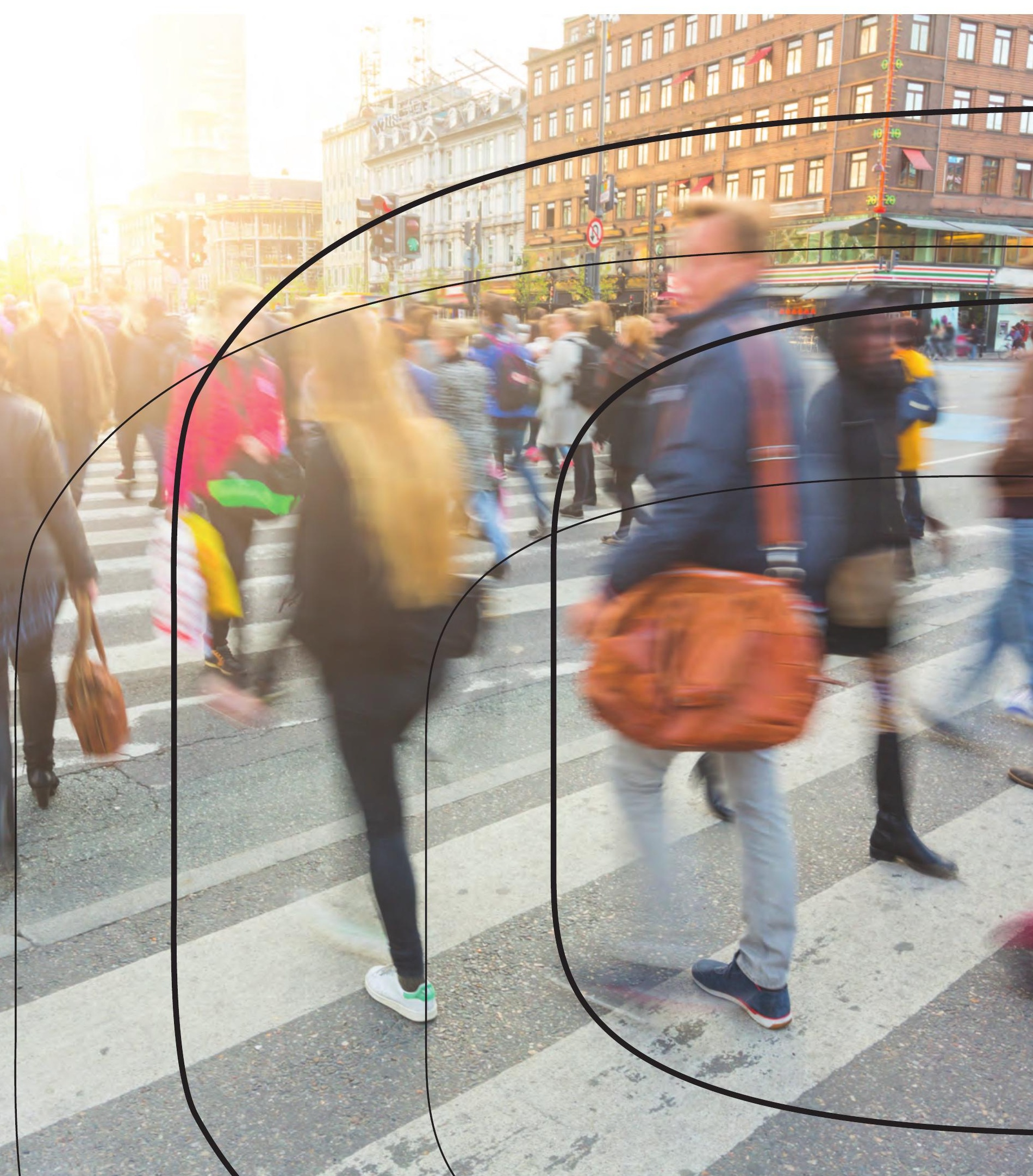



2 norden 



\title{
Place, (In)Equality and Gender
}

\author{
A Mapping of Challenges and Best Practices \\ in Relation to Gender, Education and \\ Population Flows in Nordic Peripheral Areas
}

Stine Thidemann Faber, Helene Pristed Nielsen and Kathrine Bjerg Bennike 
Place, (In)Equality and Gender

A Mapping of Challenges and Best Practices in Relation to Gender, Education and Population Flows in Nordic Peripheral Areas

Stine Thidemann Faber, Helene Pristed Nielsen and Kathrine Bjerg Bennike

ISBN 978-92-893-4267-4 (PRINT)

ISBN 978-92-893-4269-8 (PDF)

ISBN 978-92-893-4268-1 (EPUB)

http://dx.doi.org/10.6027/TN2015-558

TemaNord 2015:558

ISSN 0908-6692

(C) Nordic Council of Ministers 2015

Layout: Hanne Lebech

Cover photo: ImageSelect

Print: Rosendahls-Schultz Grafisk

Printed in Denmark

This publication has been published with financial support by the Nordic Council of Ministers. However, the contents of this publication do not necessarily reflect the views, policies or recommendations of the Nordic Council of Ministers.

www.norden.org/nordpub

\section{Nordic co-operation}

Nordic co-operation is one of the world's most extensive forms of regional collaboration, involving Denmark, Finland, Iceland, Norway, Sweden, and the Faroe Islands, Greenland, and Åland.

Nordic co-operation has firm traditions in politics, the economy, and culture. It plays an important role in European and international collaboration, and aims at creating a strong Nordic community in a strong Europe.

Nordic co-operation seeks to safeguard Nordic and regional interests and principles in the global community. Common Nordic values help the region solidify its position as one of the world's most innovative and competitive.

\section{Nordic Council of Ministers}

Ved Stranden 18

DK-1061 Copenhagen K

Phone (+45) 33960200

www.norden.org 


\section{Contents}

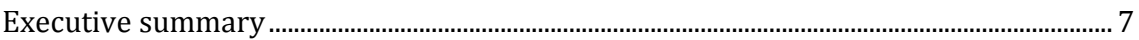

Gendered challenges in Nordic peripheral areas............................................................... 7

Themes and trends accross the Nordic countries ................................................................. 9

Preface

Introduction .................................................................................................................................21

A gendered perspective on the challenges in Nordic peripheral areas......................22

What and whom does the mapping focus on? .................................................................26

Arrangement and completion of the mapping ..............................................................30

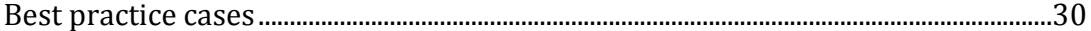

The structure of the report.................................................................................................31

1. Living Conditions, Demographic Transformations and Gender.........................................33

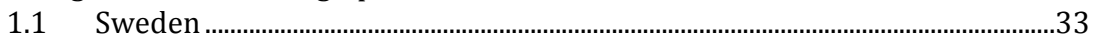

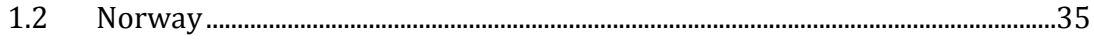

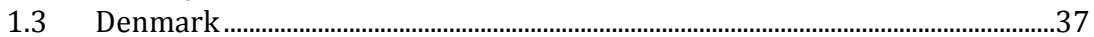

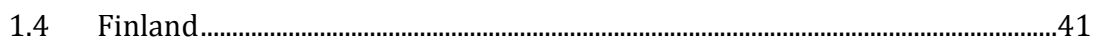

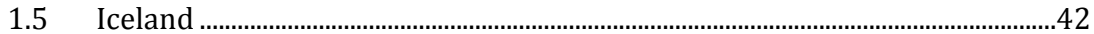

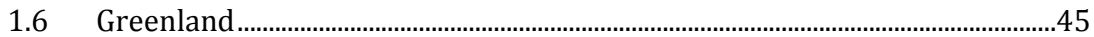

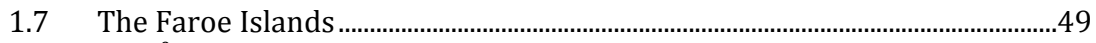

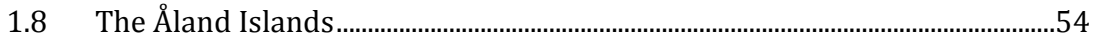

2. Education, (Im)Mobility and Gender ...........................................................................59

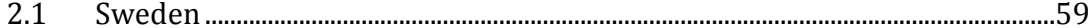

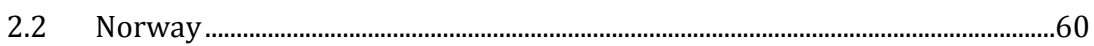

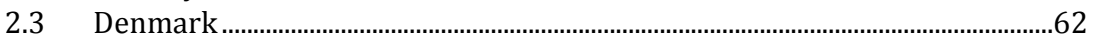

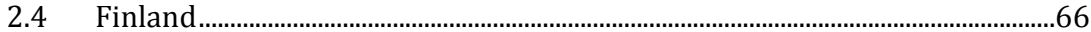

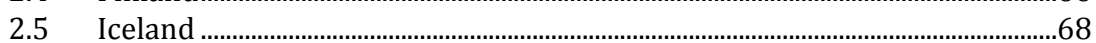

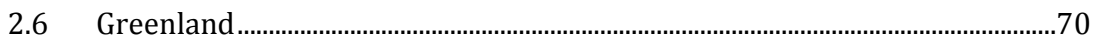

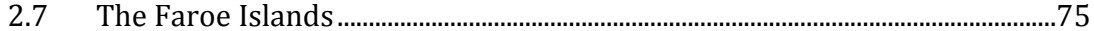

2.8 The Åland Islands...........................................................................................................7

3. Place Attachment, Everyday Life and Gender...................................................................83

$3.1 \quad$ Sweden ......................................................................................................................

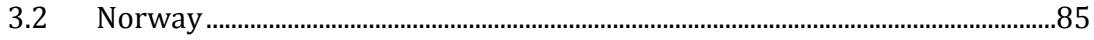

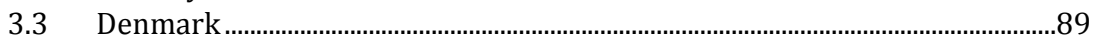

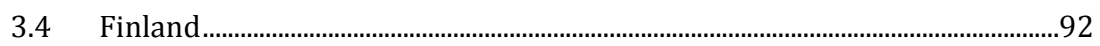

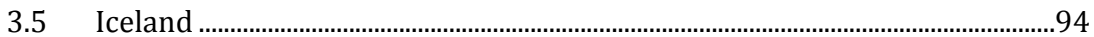

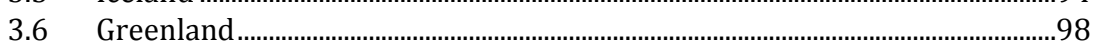

3.7 The Faroe Islands ................................................................................................ 100

3.8 The Åland Islands..................................................................................................103 
4. Gender, Work and Working Life in Transition ..............................................................111

$4.1 \quad$ Sweden ..............................................................................................................11

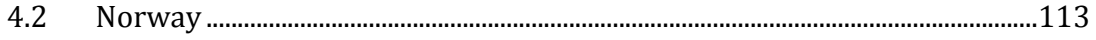

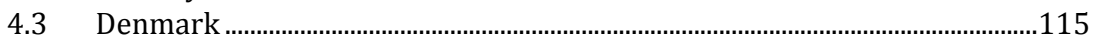

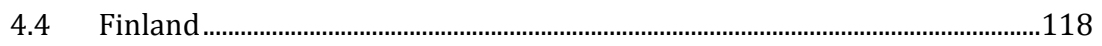

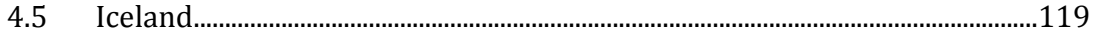

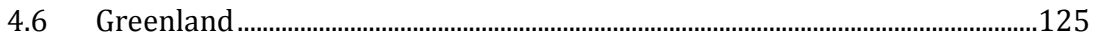

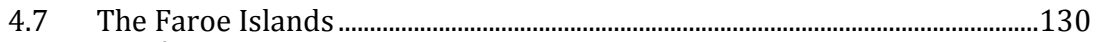

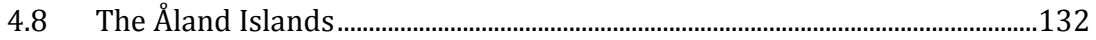

5. Changing Gender Relations and Gender Norms .........................................................141

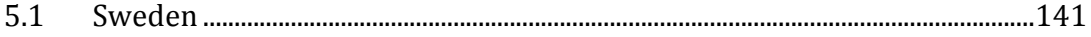

5.2 Norway ..........................................................................................................................144

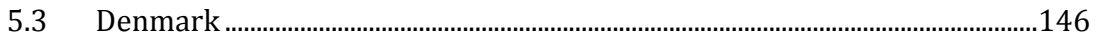

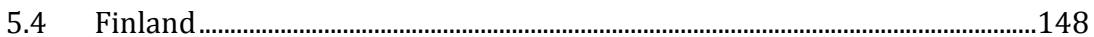

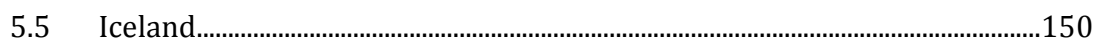

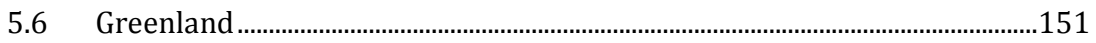

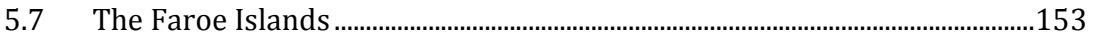

5.8 The Åland Islands .............................................................................................154

6. Young Men and Masculinity/-ies in Peripheral Areas.................................................159

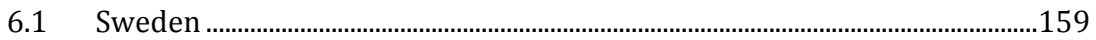

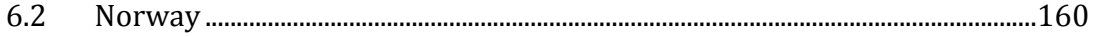

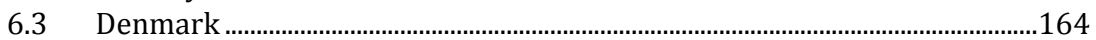

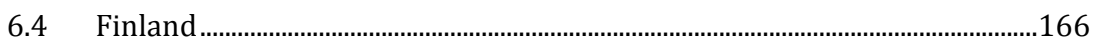

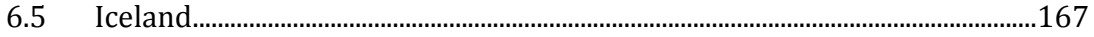

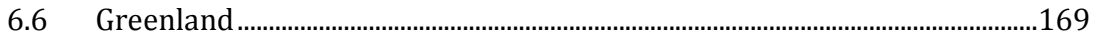

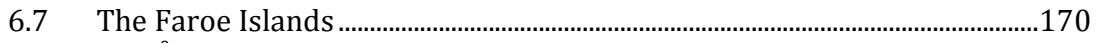

6.8 The Åland Islands ..............................................................................................172

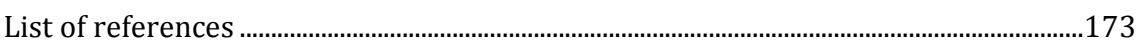

Sammenfatning....................................................................................................................185

Kønnede udviklingstendenser i Nordens yderområder ...............................................185

Udvalgte temaer og tendenser på tværs af Norden ........................................................187

\section{Overview of best practice cases in the report}

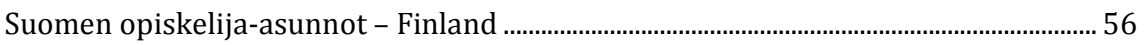

The Special Initiatives Zone - Norway ................................................................................... 57

West Nordic Master - Faroe Islands, Iceland, Norway and Greenland ............................... 80

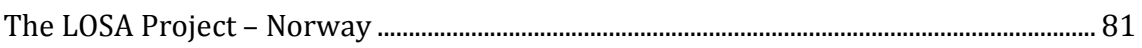

The Distance Learning Centre in Vágur - Faroe Islands...................................................106

Råd \& Dåd - Denmark - Denmark..........................................................................................107

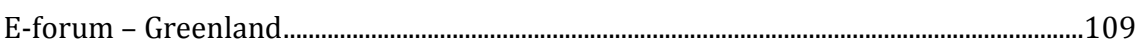

The NITUS Project - Sweden ............................................................................................110

Project Qualifying for new jobs - Denmark ........................................................................139

Brautargengi - Project for female entrepreneurs - Iceland .............................................141

North Atlantic Law Programme - Denmark, Faroe Islands and Greenland...................158

Project Men in Health - Norway .......................................................................................159 


\section{Executive summary}

This report presents the results from a cross-Nordic mapping of existing research and literature on gender, education and population flows in the peripheral areas of Norway, Denmark, Sweden, Finland, Iceland and the autonomous territories of Greenland, the Faroe Islands and Åland. The Nordic Council of Ministers and the Danish Ministry of Children, Gender Equality, Integration and Social Affairs commission the mapping.

The purpose of the mapping has been to highlight development trends and identify best practice cases across the Nordic region's peripheral areas, and thus contribute to the exchange of knowledge and create a basis for joint talks and discussion. A pivotal aim of this mapping has been to shed light on the challenges facing the peripheral areas, while at the same time focusing on the intersection between gender and place/space. As stated in the mapping, the places where men and women live, and the spheres they move around in (e.g. in the family, in the educational institutions, at the workplaces, etc.) are all rooted in specific geographical locations, that contribute to define both which opportunity structures exist, and the degrees of freedom each individual face. Thus, the mapping is based on an understanding that gender must be taken into consideration, if one aims to fully understand trends in the peripheral areas of the Nordic countries.

\section{Gendered challenges in Nordic peripheral areas}

The mapping sheds light on a number of specific developments which are of particular importance for the viability and cohesion of the peripheral areas, and for the people who live there. This applies, for example, to challenges related to changing living conditions, stagnant or negative economic development and low growth, a decline in the number of jobs (especially in the traditional male professions), and not least migration and depopulation. The fact, that women are more likely than men to leave the Nordic peripheral areas and move to the cities, has given rise to concepts such as female exodus (kvindeflugt) and female deficit (kvindeunderskud). 
As stated in the mapping, the fact that women in great numbers choose to move from the less populated areas in the Nordic countries towards the cities is not a new problem; in fact, for over 100 years, research has pointed to this trend, although global developments in recent times seem to have contributed to intensify the negative trends. At the same time, in recent years there has been an increased focus on the men being left behind, and it has been argued that the restructuring of the labour markets in the peripheral areas (and a significant decline in the so-called "male professions") has put the more traditional forms of masculinity under pressure. The men living in the peripheral areas have, in this debate, been described as reluctant towards transitions, uneducated, marginalised and as losers, who are not able to keep up with the pace of contemporary modern societies. Thus, in recent years, we have witnessed a shift, where it is not only articulated as a problem that the women in the peripheral areas of the Nordic countries choose to move i.e. that they disappear from the areas - but now it is also articulated as a problem that the men in these areas choose not to move. It is on the basis of this issue that this report uncovers some of the aspects related to the intersection of gender and place/space, and this includes mapping existing research and literature.

Above all, the mapping uncovers the challenges related to education, work and leisure. As shown, the described challenges hold many dimensions - both at the level of the individual, the collective and society, and in the interaction between the levels.

Across the different Nordic locations there are in general a number of relevant similarities. This is reflected in the educational system, on the labor market and generally in the local communities of the Nordic peripheral areas. However, when one considers the Nordic countries as an entity, there are also a number of contrasting differences, which contributes to make the challenges that this mapping tries to uncover even more complex. For instance these differences relate to geographical distances, population density, distribution of natural resources, organization of the social infrastructure, the speed at which the national, regional and local education systems and labour markets are changing, differences in the characteristics of these labour markets, and the sectors that dominate.

In the case of Iceland, the Faroe Islands, Greenland and the Åland Islands, these are small island communities with noticeably fewer inhabitants than in the other Nordic countries. This has implications for the nature of the changes, as well as the discussions on how they can be countered. Yet, also in these places it applies that the developments hold a clear gender dimension. 


\section{Themes and trends accross the Nordic countries}

In this chapter of the report, it is important to emphasize that the format of an executive summary does not leave very much room for differences, variations and nuances; neither as regards differences and variations within the different Nordic countries, nor in relation to the inclusion of variations and nuances in terms of gender. Unfortunately, this is an aspect that is hard to avoid when you summarize, especially when the subject has been so broad and comprehensive, as is the case in this mapping. We would therefore like to stress that the individual chapters of the mapping ought to be read in order to obtain a thorough understanding of the interrelations between gender, education and population flows in the Nordic peripheral areas.

Nevertheless, despite the reservations outlined above, below we attempt to summarise a series of recurring themes and trends, identified through the mapping of existing literature in the fields.

\section{There are national variations in the type and amount of literature on the subject}

The mapping aimed to identify research and literature on gender, education and population flows in the Nordic countries. Our desk research shows that there are national variations in the type and amount of literature. There is, for instance, clearly a rich field of researchers in Norway who are engaging in education, population flows and problems of the peripheral areas - and to some extent also with a gender angle - while in other Nordic countries it has been more difficult to identify literature on the subject.

\section{There is a lack of research and literature with a gender perspective}

On the basis of the identified literature, we note that gender as a category is absent in much of the literature on education and population flows in Nordic peripheries. The literature, which includes the work of educational researchers, regional/rural researchers, etc., is often based on youth and education in general, or on general peripheral area problems; and is often presented without consideration of differences and/or similarities between boys/young men and girls/young women. Gender analysis is thus non-existent in a big part of the literature on the subject, or it is at least often downplayed, while other categories (such as ethnicity or social class or even completely different categories, such as "preparedness for education" or "vulnerability") are given more attention. This tendency is not only visible in research, but it also applies when gender, education and population flows is discussed among political actors or in 
practice (e.g. in the educational institutions). Also here, gender is rarely discussed to a significant degree; often because gender is construed as irrelevant, or because gender is understood from a biological perspective, i.e., it is considered as "natural" intrinsic properties of individual young people, and is thus not considered susceptible to change.

\section{The developments in the Nordic peripheral areas cannot be analyzed nor solved without taking the gender perspective into account}

Yet, gender is not just something you "are" or "have", but also something which is "done". Gender is, in other words, also about social actions, about ideas and expectations as well as opportunity structures, and that is why the developments of the Nordic peripheral areas contain - as the mapping also illustrates - dimensions that cannot be analyzed nor solved without taking the gender perspective into account. These developments for instance revolve around changed local business traditions and education patterns in the peripheral areas, challenges regarding transportation, social infrastructure, local attachment patterns or a lack thereof, labour market segregation, change in recruitment patterns, etc. As a result of processes linked to structural, economic and socio-cultural changes, the literature shows that the peripheral areas face a number of challenges. While some adapt to the new conditions, others are left behind, and as the mapping illustrates, particularly the men seem to be affected by the developments in the peripheral areas.

\section{The young people in the peripheral areas feel a huge pressure to leave their local communities}

The literature shows that the young people, depending on where they live, make their choices based on very different terms, because the young people in the Nordic peripheral areas often either are outright forced to, and/or feel a huge pressure to leave their local communities. In other words, you can say that the youth in the Nordic peripheral areas feel the changed patterns of education and employment, as well as the increasing demands for mobility particularly strongly. In contrast to the young people who live centrally, the young people in the peripheries - and especially the women - feel a pressure to leave their local communities. In certain peripheral areas, the young people do not even have access to neither a secondary education nor higher education, or they only have access to a limited choice of education programs and/or fields of study, which in practice may mean that they are forced to leave home at quite an early age. Previous studies show that it is especially the young women in the peripheral areas who move away 
from home at a young age. However, it must be said here that there are different traditions across the Nordic countries; for example, in both Greenland, Iceland and the Faroe Islands, it is tradition that the young people leave the places and go abroad (primarily to Denmark) - to get an education. Yet, where a big part of the Greenlandic and the Faroese youth do not return home again, there is a larger tradition in Iceland of young people returning home, and using their acquired education here. The identified literature thus stresses that young people of both sexes leave their homeland in the peripheral areas, not only because they want to see and experience the world or because the act of moving out from home is a natural part of growing up, but also because it feels impossible for them to stay, either due to a lack of education and employment opportunities, or because they find the local communities of the peripheral areas limiting or claustrophobic.

\section{The literature shows that it is often education or work-related when young people (especially the women) move away from the peripheral areas}

Furthermore, the mapping also reveals that young people in the Nordic peripheral areas have different aspirations as to whether in the longer term there will be a place for them in the location where they live. This is precisely where the literature shows that gender appears to play an important role, since it is pointed out across the Nordic literature that the changed conditions in the peripheral areas means that the young women experience to a larger degree that there are limitations associated with their gender. This seems, to a larger extent than is true for the young men, to collide with the young women's wishes as to how they would like to live in the long term, and what is actually possible in the places where they live. It is thus clear from the mapping that the young women in the peripheral areas seem to focus more on higher education than the young men. The literature also suggests that the women's desire to move away from the peripheral areas to some extent can be linked to a desire of the women to gain access to more and equal opportunities - in education, work and everyday life - as well as a greater degree of freedom from gender traditional expectations and norms.

\section{The place where the young people live forms the premises for their opportunities and choices in relation to education}

Although we in the Nordic countries have a political ideal of equality at the national level, there are, as the mapping indicates, context-specific variations. In this way, we can say that the norm of equal opportunities for all, as is communicated in the Nordic education systems, does not 
always correspond with the actual situation in the Nordic peripheral areas. The mapping thus indicates that across the Nordic countries it is important to ask the question of whether men and women actually really have equal access to obtain the education they may want. The place, the young people live, sets its limits on what is realistic and accessible, and thus, the place contributes to shape the opportunity horizon, which the young people relate to.

\section{The place where the young people live also forms the premises for} commitment and the desire to learn

That the places where the young people live contribute to form the premises does not only apply to young people's opportunities and choices in terms of education and employment, but to some extent also their commitment and desire to learn. In some peripheral areas, the local labour market and business sector call for less education, whereby the act of opting out of education emerges as a sensible and rational choice, or the culture of the educational institutions is experienced by some students as "foreign", because their identities are rooted in a local community, where the focus is on other values and forms of cultural/social capital, than those that are valued within the education system. In the literature, this is referred to as a cultural collision experienced by the young people in the peripheral areas, especially the young men; a collision between, on the one hand, the young men's values and perception of what constitutes "a good life", and, on the other hand, the educational and career-oriented narrative (discourse) which is raised inter alia in the media and by politicians on the national arena - consolidating that you "only matter, if you accomplish something" - or that you are superior and you get the furthest, if you pursue an academic education.

\section{Geographical mobility is often linked to social mobility}

As shown by research in this area, education is central to the life course of young people, both in terms of adjustments to the surrounding labour market, and way of life and identity. For the young people from the Nordic peripheral areas and from the smaller urban communities in these areas, choosing a higher education often implies moving away from home. At the same time, geographic mobility is in many cases also linked to social mobility, as a part of the young people from the peripheral areas come from homes and/or local environments where pursuing a higher education has not been customary. 
The young women in the periphery feel that they have fewer opportunities on the local labour markets, in the local associations, as well as in recreational and cultural activities

The literature across the Nordic countries highlights that the young people in the peripheral areas overall are subject to a number of structural conditions, not only in connection with their choice of education, but also regarding business and professional life (access to the labour market, geographical distances, infrastructure, internship opportunities, etc.). When the young women in the periphery often find that they have fewer opportunities than the young men, it is partly because the job opportunities in the peripheral areas (often in the primary sector) are not available to them to the same extent. Yet, there are also other aspects that are emphasized in the research on the subject; the type and degree of an associational life, recreational and cultural activities play an important role in the young peoples' choices of either staying in the peripheral areas or moving away and here the literature suggests that the activities which the young men participate in appear to attach them more to the place than the activities which the young women participate in.

\section{Gender relations and gender norms are an important part of the youth's view on the local labour market and on their sense of place attachment}

The local (and regional) labour market in the peripheral areas forms a framework which the young people assess their options against. The literature on the subject suggests that it is not only the educational opportunities, but also the opportunities on the labor market, which affect the youth's, particularly the women's, desire to move away or - in the long term - to return home. Gender segregation in educational choice as well as in labour market participation is pronounced in the Nordic peripheral areas. There are indications that more and more young women break with the traditional boundaries/expectations, and thus set examples that others can be inspired by, yet the question is whether young men in the peripheral areas are equally encouraged to choose unconventionally? In any event, the literature indicates that there is a tendency that the young men have a greater sense of place attachment than the young women. This may be because young men more often identify with the types of occupations and lifestyles which have historical and geographical roots in the local areas of the periphery. 


\section{It is important to be able to offer more varied opportunities and types of work in the peripheral areas}

When research on the subject shows that for the youth, particularly the women, it is especially the lacking education and job opportunities which explain why they want to move away, this points to the importance of offering more varied opportunities and types of work in the peripheral areas. In order to ensure that more of the young women choose to stay or choose to return to their local communities after graduation, it seems specifically necessary to create local employment opportunities in the peripheral areas which require higher education.

\section{The strong gender segregation of the Nordic labour markets reinforces the negative developments in the peripheral areas}

A common feature across the labour markets of the Nordic countries is a strong tendency to gender segregation - this is more pronounced in certain countries rather than others, but there is nevertheless a general tendency for women to be working in the public sector, and men in the private - and that particularly the primary sector (agriculture, forestry, and mining) is largely dominated by men. This gender segregation of the labour market has been a contributing factor to the fact that particularly the men have been hard hit by the financial crisis in several of the Nordic peripheral areas, as the number of traditional male jobs has decreased significantly in several places. The mapping of the literature regarding developments in the labour markets in the peripheral areas stresses that an important key to creating change in the education patterns and the population flows may lie in the blurring of distinctions between notions of "male jobs" and "female jobs". If the local labour markets would succeed in breaking these associations, it would both expand the supply of (experienced) employment and education opportunities for both young men and women, and also contribute to the needed break with traditional gender norms, which the young women otherwise seek to get away from through their relocations to urban areas.

\section{The peripheral areas contain potentially inherent patriarchal structures that push the young women away}

In some of the literature, it has been highlighted that the peripheral areas tend to push women away, partly because of their inherent patriarchal structures. Thus, a part of the literature concludes that several of the young women perceive the act of moving away from the small local communities in the peripheral areas and pursuing an education, as crucial to shaping their own identity and creating a lifestyle which is uncoupled from local cultural and structural limitations; including gen- 
dered expectations of women's roles/responsibilities, relationships and motherhood - gendered expectations that these young women perceive as limiting their space for action. Our desk research points to a lack of research about the unwritten rules and norms which govern the relations between men and women in the peripheral areas.

\section{Some men make a conscious choice to stay in the peripheral areas because they thrive there}

In the discussion of the female deficit that characterises many of the Nordic peripheral areas, it is also relevant to emphasise that it is not just about enticing newcomers (especially women) to come to the peripheral areas, but that it can also be about protecting those (especially men), who remain there - and in extension of this: to highlight more positive images. The tendency of the young men having a greater degree of place attachment than the young women is not just about a particular type of life pattern, about professional communities related to the local business traditions, or about the leisure offers in the peripheral areas harmonizing well with traditional male interests - according to the literature, it is also about the young men staying in the local communities because they prefer life in the country side, and because they feel that there are some values that they only/best can practice here. The idea that the men staying in the peripheral areas may happen to have made conscious and deliberate choices regarding residence, education and occupation is included to a very little extent as an element in the cross-Nordic debate.

\section{Some men in the peripheral areas are particularly affected by the restructuring of the local labour markets and the changing living conditions in the peripheral areas}

The literature review shows that the men appear to be particularly affected by the restructuring of the labour market and the changing conditions of life, which characterise the peripheral areas in all the Nordic countries. Moreover, it has been highlighted that the recent years' financial crisis has amplified this development, which is why - from a gender perspective - one does not talk about the recession, but the mancession. This should be seen in conjunction with the current strong focus on mobility and flexibility, which, according to the research literature, contributes to create a rather negative story about the men "staying behind" in the peripheral areas. The literature suggests that there are men in the peripheral areas who are experiencing ambivalence - and some also exhibit signs of what has been described as melancholic masculinity within the research literature. This should be seen in relation to the fact that many of the young men in the peripheral areas have grown up with 
a traditional understanding of gender, where masculinity and hard physical labour are closely linked. With the restructuring of the local labour markets, and the rising unemployment in the traditional male professions, this understanding is challenged.

\section{The negative stigma of the peripheral areas affects the young people of both sexes}

From the mapping it also becomes clear that there are various negative notions about, and articulations of, the Nordic peripheral areas, and the people who live there. This negative stigma is not only rooted in the media; it is also frequently heard among politicians, practitioners, researchers and, not least, it is heard by the people, especially the youth, who live in the periphery. Within research, this is referred to as the socalled the supra-local flows; these include images of the characteristics of "the good life" and stories related to the peripheral areas, which are shown in both print, digital and social media and on the web in general. Precisely the Internet and the social media are important arenas in the young people's daily lives, and also for many young people in the peripheral areas they are an important frame of reference for the way they perceive themselves and the thoughts they have about their future

\section{New stories need to be told: Peripheral areas as places to be nurtured and preserved?}

According to the identified literature, the negative stigma of the peripheral areas play quite a central role with regards to the trends that the mapping focuses on - yet, the mapping emphasises that there is a the lack of further knowledge about this dimension, and any potential gender differences related to this. There are indications that in the peripheral areas, young people of both genders have a negative assessment of their own local area and the youth here, and especially the young men apparently experience that it is expected that they move away in order to not be/appear to be backward (to be untrendy). The negative stories about the Nordic peripheral areas indicate that they are perceived as places that must be changed (modernised), rather than places to be nurtured and preserved. Here, the research indicates that the perception, which the young people obtain, of the peripheral areas is more important than what these places are actually like. The literature has pointed out that by furthering other images and opportunities, one can contribute to the development of new patterns and strategies. 


\section{Best prattice cases}

As part of the mapping, we have identified number of best practice cases. The best practice cases, which are presented troughout the report, are examples of actions or activities that appears to have a cross-Nordic potential for solving a range of the different types of challenged in the Nordic peripheral areas that have been identified through this mapping. 



\section{Preface}

The purpose of this mapping is to shed light on challenges and best practices in relation to gender, education and population flows in peripheral areas in the Nordic countries. The mapping is intended to contribute to an exchange of experience across the Nordic region and be a source of inspiration for decision-makers and practitioners with an interest in the subject, as well as students and researchers in the field.

The mapping is compiled by Centre for Equality, Diversity and Gender (EDGE) at Aalborg University, Denmark, and is carried out on behalf of The Nordic Council of Ministers. The mapping has been completed during the period January to May 2015.

The report presents a selected overview of existing research and literature on gender, education and population flows. This is a research area that is very wide and with strands into many different and diverse academic traditions. The principle which has been applied in the identification of relevant literature, however, has been to focus on contributions from gender research, or other types of contributions which include a gender perspective, as the aim has been to illustrate the importance of gender in relation to development trends in the Nordic peripheral areas.

Work on the mapping been ongoing in dialogue with the Nordic Council of Ministers and the Ministry of Children, Equality, Integration and Social Affairs, Denmark, and we want to thank both parties for good and constructive cooperation.

We also thank the reference group, which has contributed input to the report, consisting of Associate Professor Gestur Hovgaard at the Faroese University, who has contributed knowledge particularly on Faroese conditions, Professor Gry Paulgaard at UIT - Norway's Arctic University for contributing with her knowledge, particularly on young people in Norway's peripheral areas, and last but not least, Senior Researcher at Nordregio Rasmus Ole Rasmussen, who has specifically provided input on sections of the report relating to Greenland.

In our efforts to retrieve information for the report, we have also received assistance from a number of Nordic research colleagues and from various gender research centres throughout the Nordic region. A big 
thank you to all for the great hospitality and the many inputs we have received along the way.

For this English version of the report, special thanks are also due to Ida Jessen, Anita Nissen, Pernille Budde Haensel, Kirsten Gammelgaard and Louise Hartmann for invaluable assistance.

The undersigned have authored the report and have been responsible for the identification of relevant literature and best practice cases. Ultimately, the content of the report, with any remaining deficiencies, is entirely our responsibility.

Aalborg University, EDGE

Associate Professor, Stine Thidemann Faber

Associate Professor, Helene Pristed Nielsen

Research Assistant, Kathrine Bjerg Bennike 


\section{Introduction}

The Nordic countries are faced with a series of challenges often particularly accentuated in the peripheral areas of the regions. The circumstances relate to, for instance, altered living conditions caused by global changes, stagnated or negative economic development - including low growth, decrease in the amount of workplaces (particularly in the traditionally male-dominated professions) as well as, not least, migration and depopulation which is partly due to the fact that the young people of the area (especially the women) move to bigger cities to educate themselves. The challenges in question are not only significant in relation to the viability and cohesion of the areas, but also for the men and women who live there and their mutual social relations.

The overall purpose of the mapping is from a gender perspective to clarify the specific challenges the peripheral areas of the Nordic region are faced with and to identify best practice cases in regards to gender, education and population flows across the Nordic countries. These best practice cases have been collected with a particular view to finding examples of practices that are deemed to be operable in other Nordic contexts than those which they stem from. We have also endeavored to find examples that are related to the different themes in the report and for the same reason, the various best practice case studies are presented at the end of each thematic chapter of the report.

In the report we use the term peripheral areas even though such a term is debatable. As Ebbensgaard and Beck (2009) point out, such a term indicates a scale, whereas what is characterized as centre and what is characterized as periphery often can and will be defined differently depending on the eye of the beholder. When the mapping uses the term peripheral areas, the purpose is not to support or reinforce potential existing perceptions of certain geographical areas as less desirable, but, however, to shed light on real existing differences in terms of living conditions, and material as well as immaterial aspects of life which play a prominent role in the peripheral areas of the North. For this English version of the report, we have chosen the phrase "small village" as a translation of the Nordic phrase "bygd", although the former admittedly does not quite capture the connotations inherent in the latter. 


\section{A gendered perspective on the challenges in Nordic peripheral areas}

Within research, it has been emphasized that the adjustment to a more global labour market means that flexibility and mobility are becoming important key words particularly for the Nordic peripheral areas, where demands and expectations in the individual local communities are undergoing changes. While some adjust to the new conditions (e.g. women who find new jobs in, for instance, the service sector), others are left out (e.g. low-educated men whose qualifications are no longer in demand on the new labour market) (Faber and Pristed Nielsen 2005). The men in the Nordic peripheral areas seem to be particularly affected by the process. The fact that the financial crisis of the past years has furthered this process has been underlined. Thus, seen from a gender equality perspective, the financial crisis is referred to as "the mancession" (as opposed to "the recession") as the crisis particularly has put pressure on the traditionally male-dominated professions (Weyhe 2011:248).

Especially the young in the Nordic peripheral areas experience the challenges because of the changed patterns of education and employment combined with an increasing expectation and demand for mobility. As opposed to the young, who are centrally located, the young of the Nordic peripheral areas feel pressured to leave their local communities, and research points to the fact that it is particularly the young women who relocate. And this greatly affects the Nordic peripheral areas both because of the amount of women that relocate, but also due to the consequent drop in birth rates (Svensson 2006, Rahut and Littke in press). This development provides a breeding ground for further negative development trends. If the development continues, a large residual group of Nordic men will be left behind and remain in the peripheral areas with no education, no job and no women (Skrede 2004; Plambech 2005; Rauhut et al. 2008; Bærenholdt and Granås 2008).

The mapping includes, as a starting point, all of the Nordic countries - Norway, Sweden, Finland, Iceland and the self-governing areas: Greenland, the Faroe Islands and the Åland Islands - however, ${ }^{1}$ with more consideration to some countries rather than others when shedding light on overall common development trends.

${ }^{1}$ The Åland Islands are only covered to a smaller extent as the literature on the topic is fairly limited. 
In overall terms, there is a list of common features across the different Nordic locations which make comparing discussions of different gendered development trends possible. The Nordic countries are not only tied together by history, but do also, to a large extent, share common ground in terms of politics, culture and values (Helvé et al. 2003; Jákupsstova, Sølvará and Hovgaard 2014). This is, for instance, seen in the construction of welfare society and in types of welfare benefits. Even though it differs how these aspects are handled in the individual Nordic countries, the similarities are so great that it is common to refer to "the Nordic model of welfare". The Nordic countries are also historically known for a high level of equality between genders and, compared to a number of other European countries, to focus greatly on equal opportunities for men and women/boys and girls. This is manifested in the educational system, on the labour market and generally in the Nordic societies.

When considering the Nordic countries as a unit, there is also however a list of contrasting differences contributing to make the challenges, which this mapping focusses on, even more complex. The differences relate to, by way of example, geographical distances, population density, distribution of natural resources, the rate at which education and labour markets change, differences in the characterisation of the local labour markets and which sectors are dominating, organisation of the social infrastructure and, finally, types of local trade and industry, association activities and occupations. In the light of this, there are obviously variations in the political initiatives across the Nordic countries. Additionally, when it comes to Nordic education policies, national differences are also evident. In Sweden and Norway they have worked politically on promoting gender equality by, to a greater extent than what is seen for example in Denmark, integrating equality of status in the educational goals and in the curricula and by initiating and encouraging local development work, etc. (Reisby and Knudsen 2005).

In relation to the mapping, it is, on the one hand, relevant to point out that the peripheral areas in the Nordic countries are faced with a line of similar challenges. Such challenges do, for instance, regard altered local professional traditions, gender-specific choices of education, challenges relating to transportation to and from the educational institutiona, patterns of local attachment or the lack of such, gender-segregation of the labour markets, changes in recruitment patterns, etc. On the other hand, it is also important to point out that the national differences open up for a nuanced discussion of which experiences are tied up with specific local conditions, ultimately making them harder to convert, and which expe- 
riences potentially can be transferred from one Nordic context to another, enabling learning through best practices.

In the case of Denmark, Norway, Sweden and Finland, the mapping will basically focus on research and literature concerning problems related to specific, and often the least populated, peripheral areas. However, in regards to Iceland, Greenland, the Faroe Islands and the Åland Islands, the mapping of research and literature distingsuihes to a lesser extent between the regional/local variations internally in the countries, however, still with a focus on differences between rural and urban, centre and periphery.

There is no doubt that the geographical distances are far more distinctive in Norway and Sweden compared to, for instance, Denmark. This is likely to be the explanation as to why these countries have a stronger research tradition when it comes to illustrating young people's place attachment, and what significance it might have in relation to choice of education (for summary, see e.g. Karlsen and Paulgaard 2012). In terms of both the Faroe Islands and Greenland (and also the Åland Islands) population flows in relation to choice of education often entails national relocation - resulting in a drop in birth rates because many never return. As an example, the number of women of childbearing age has decreased with 19 per cent in the Faroe Islands since 1990, and, in the long term, the tendency is estimated to pose a threat to the Faroese society as a whole (Hamilton et al. 1996; Hovgaard 2015). In contrast, when it concerns the young of Norway, Sweden, Finland and to some degree Denmark and Iceland, it is more a question of relocating internally in the countries and/or commuting in order to educate oneself however, still with quite significant differences relating to distances, accessibility and time spent on transportation. In certain peripheral areas, the young do not even have access to secondary school, or they only have access to a limited selection of secondary schools that, in practice, can mean that they are forced to move away from home at a rather young age. Earlier studies show that it is the young women in the peripheral areas in particular who are likely to move away from home at an early age, which stresses the fact that there are significant gender differences among the young in the periphery compared to the young from urban areas, where the variation based on gender in regards to the act of leaving home is rather small (Roals $\varnothing$ 1997) (see also Helvé et al. 2003). The fact that women to a larger degree than men leave the peripheral areas for the cities has given cause for terms 
as e.g. female exodus (kvindeflugt) and female deficit (kvindeunderskud) (Giskeødegård and Grimsrud 2014). ${ }^{2}$

The mapping focusses on a series of problems that have significant impact on the vitality and cohesion for the peripheral areas and the people who live there. In the uncovering of research and literature within the field, the problems concerning education/supplementary training, labour and leisure time have been prioritised. Furthermore, apart from these dimensions, the literature points to the fact that there are different conceptions of, and how we speak of, life in the Nordic peripheral areas (see also Helvé et al. 2003). From a positive perspective, the peripheral areas are often presented as locations with open spaces, with possibilities of free scope for self-expression, safe environments, and tightknit social relations. From a negative perspective, the peripheral areas, and the people located there, are presented as underdeveloped, immature, ignorant and stagnant (Karlsen 2001; Paulgaard 2006 and 2015; Bloksgaard, Faber and Hansen 2014). Particularly the men in the Nordic peripheral areas seem to be referred to rather ruthlessly; that is, as people who are reluctant to change, uneducated and often as unable to provide for themselves (Bye 2009 and 2010; Eriksson 2010; Bloksgaard, Faber and Hansen 2013). This is, for instance, reflected in the fact that the men in the peripheral areas are perceived as less attractive as spouses (Plambech 2005; Gaini 2010).

Also beyond the Nordic context, the debate of the men staying behind in the periphery is increasing, and, on a regular basis, it is stated that the restructuring of the labour market (and a pronounced decrease in the traditionally male-dominated professions) has put pressure on the traditional sense of masculinity. The book Masculinity beyond the metropolis (Kenway et al. 2006) describes how young men in Australian peripheral areas view masculinity as closely related to manual labour and connected to the perseverance of the industrial worker, and this leads to, it is concluded, that this group of men experience a feeling of emptiness and potentially develop a "melancholy" (retrospective) masculinity (see also McDowell 2003; Ní Laoire and Fielding 2006; Corbett 2007 and also Kelly 2009).

\footnotetext{
${ }^{2}$ In a way, it is not a new problem that the women in the less populated areas in the Nordics leave for the cities. This has long been the case; as a matter of fact, research has been pointing out this problem for more than 100 years, partly based on census data in Great Britain at the end of the nineteenth century (Ravenstein 1885)(see also Bjarnason and Thorlindsson 2006; Giskeødegård and Grimsrud 2014).
} 
In a time where the peripheral areas are generally marked by migration, particularly among the young women, the ones that remain are often presented, by the media and in the general public debate, as being less future oriented and less adaptable than those who relocate. The negative stigma tied to the peripheral areas is not only embedded in the media, it is also regularly heard among politicians, practitioners, researchers, and, not least, registered by the people, particularly the young, living in the peripheral areas. The negative stigma of the peripheral areas will only to a minor extent be referred to in the mapping, even though it, according to for example Helvé et al. (2003), plays a key part in relation to the development trends the mapping focusses on. In a cross Nordic report on young people, the potential consequences of the negative stigma of the peripheral areas are described as follows:

People have a tendency to act upon their perception of reality rather than on how it actually is. The consequences of such actions may mean that rural areas and smaller towns find it even harder to survive than need be. In everyday speech this phenomenon is often referred to as the self-fulfilling prophecy (Helvé et al. 2003: 30; own translation).

As it appears, the problems that the mapping engages with contain various dimensions - both on an individual, group and societal level as well as the interplay between said levels. In addition to this, the mapping crosses a variety of research traditions. However, it is important to underline that the mapping predominantly focusses on contributions from gender research or on other types of contributions that take a gendered approach, as the task cf. earlier, specifically has been to clarify the meaning of gender related to challenges and best practices in the Nordic peripheral areas.

\section{What and whom does the mapping focus on?}

The mapping is based on an understanding that gender is individually important at different levels, just as the underlying basis is that gender does not only refer to biological differences that may play a role for individuals in relation to their construction of identity, but also that gender plays a significant role in socio-cultural terms (e.g. as ideas, metaphors and categories, as well as a principle for distribution of power, for decision making and for recognition) (Thurén 2003, Svensson 2006).

The mapping furthermore employs the understanding that places are gendered as a point of departure. To idea that places are "gendered" is 
partly about focussing on how places are embedded with meanings and relations that control behaviour and perceptions and also map out guidelines for how masculinity and femininity can be "done", but, also, how places are experienced and interpreted differently depending on whether you are a man or a woman and depending on age, social position and ethnic belonging (Faber and Pristed Nielsen 2015).

Feminist researchers have claimed that the relationship between the genders both reflect and influence the spatial organisation of society, and feminist geographers in particular have attempted to illustrate the spatial construction of gender (Faber and Pristed Nielsen 2015). This perspective has, for instance, caused Zelinsky and others to argue that we ought to analyse places and locations from a gender perspective:

The human geographer must view reality stereoscopically, so to speak, through the eyes of both men and women, since to do otherwise is to remain more than half-blind (Zelinsky, Monk and Hanson 1982: 353).

The idea of places as gendered is also a key point of departure for the American geographer Massey $(1994 ; 2005)$. She argues that places are not merely shaped by different and varied social relations between people that are stretched across time and space, but, also, that places are experienced and interpreted differently depending on whether you are a man or a woman. In a Swedish context, also Friberg (2000) and Forsberg (2002) have pointed out the close relation between gender and time/space - for instance, by calling attention to the fact that places are not just shaped by how we act as individuals, but also that places define which geographies of opportunities exist and what degrees of freedom each individual can have. Even though in the Nordics we have a political ideal about gender equality on a national scale, there will often be variations specific to the context. Stenbacka (2007) writes the following on this:

\footnotetext{
The political ideal of equality among men and women build upon existing theoretical prerequisites for gender equality, but nonetheless local gender structures still show inequality and differences in gender relations as well as in space of action for both men and women. Depending on where you are and what the local context is, men and women live under different conditions and with different expectations (Stenbacka 2007: 86).
}

The importance of clarifying the relation between gender and time/space, relates to how places not only denote specific geographical locations but also the complex social placement of individuals at the same time. The place in which men and women live and the spheres they frequent (e.g. within the family, educational institutions, workplaces, 
etc.), are all rooted in specific geographical locations that are embedded with meaning and different ideas of what is considered socially acceptable (Cresswell 1996; Hoven and Hörselmann 2005; Svensson 2006; Bye 2010). Meanwhile, it is an important point that there is no singular/unambiguous male or female identity. In an understanding that (gender) identity is something that is continuously "done" and negotiated, masculinities and femininities are therefore referred to in the plural (jf. fx Connell 1995).

As gender, education and population flows together make up the theme of this mapping, there is a specific focus on the young, on secondary education as well as further education systems in the Nordic peripheral areas. The mapping only includes research and literature on preschools (day-care institutions) and primary schools to a small degree. However, selected perspectives on supplementary and further training have been included as well as, to some extent, literature on the development trends of the labour markets in the Nordic peripheral areas.

It is important to stress that the youth in the Nordic countries is not a homogeneous group. Among these young people one finds significant differences (social, cultural and geographical) and said differences obviously affect how the young men and women decide on what kind of education and/or professional life to choose, and whether they should stay or move away from their local area. Parallel to this perspective, as will become clear in the report, is a discussion on gender relations and the significance of gender in connection to the possibilities of young people in the Nordic region as a group and as individuals.

Simultaneously, it is a key point that the young people's conditions of life and the youth's role models more than ever before are free from local limits and boundaries. Young men and women are today, across the Nordic region, exposed to numerous options and competing alternatives, and they are more than ever forced to reflect on their own disposals and possible consequences of the choices they make (Giddens 1991; Ziehe 1996). Brannen and Nilsen (2002) speak of this shift in the young people's life planning from a situation where standard biographies earlier dominating, in oppositions to a type of planning that today is better described as choice biographies. However, at the same time, they point out that any idea of young people's unstandardized and individual biographies resulting from (completely) free choices, would be a caricature. Both gender and class, but also other structural conditions such as the places where the young people grow up, shape the choice biographies and thus have great importance for the way the young people's life stories actually evolve (see also Bloksgaard, Faber and Hansen 2015). 
According to Svensson (2006), who has studied the young in the Swedish periphery, the young people have different ideas of what it means to be young nowadays. Such ideas reflect varying experiences which also give the young in the Nordic peripheral areas different expectations of whether, in the long run, there is room for them where they live. In supplement, Trondman and Bunar (2001) speak of the tragic gab that occurs between, on the one hand, the changed conditions in the local communities, and, on the other hand, the self-image and the dreams of the future that the upbringing in the peripheral areas of the Nordic youth provides breeding ground for. The tragic gab occurs on the basis of a discrepancy between how the youth in the Nordic peripheral areas see themselves and their desire for what they would like to do and become in the long run, and what is actually possible based on where they are located.

According to Bourn (2008) young people are some of those who are affected most by the economic restructuring and the social changes that came with globalization. This also applies for the young in the Nordic peripheral areas. The fact that their actions and identity formations take place in locations that are particularly under pressure because of the changed patterns for education and occupation, along with the increasing demand for mobility, result in them experiencing the consequences of globalization at closest range. Based on a study of the young in the peripheral areas of Norway, Paulgaard (2012) concludes that the changed conditions in peripheral areas in some way seems to prompt that the young people living in these areas are facing a vast number of limitations, compared to young people living in urban areas, who rather seems to be faced with a vast number of possibilities. When globalization changes the foundation of the local areas, the demand for mobility becomes particularly noticeable for the young in the Nordic peripheral areas. Moreover, even though this group of young people must have the same sense of ambition and desire to move away from home, desire to travel and/or desire to gain independence as the young in the bigger cities, for this group of young people it is not just about leaving behind their local area in a narrow sense - it is also a decision that entails leaving their parents, possibly other family and their network.

It is often related to education or profession when young women move away from the peripheral areas, however, other aspects are also accentuated in the research on the topic; among other things, it has cf. previously been brought out that the media across the Nordic countries present an image of city life as particularly desirable/trendy, and that a result of this is that the young women are drawn to the cities (Gunnarsson 1993; 
Karlsen 2001; Paulgaard 2006). It must, however, be added that Dahlström (2006) has concluded that the goal for young women, who has been bought up in the peripheral areas, is actually not to move to the urban areas, but rather to move away from the periphery. Based on this, Dahlström concludes that the attraction force of the urban areas is overrated - nonetheless, she assesses that this does not change the fact that the peripheral areas have a tendency to push the women away due to, for instance, their inherent patriarchal structures (see also Rauhut and Littke 2014) (will be elaborated more later). Additionally, Dahlström points out that the educational systems in the Nordic countries communicate a standard of equal opportunities for all; a message which does not correspond with the actual situation in the Nordic peripheral areas.

\section{Arrangement and completion of the mapping}

The mapping is completed as a desk research study based on existing literature from the entire Nordic region including previous reports and evaluations written in the context of The Nordic Council of Ministers, among others. The purpose of the mapping is cf. earlier to present a selected presentation of existing research and literature on gender, education and population flows as well as uncover central perspectives and central contributors within this topic.

As part of the desk research, a survey inquiry has been completed among Nordic researchers, professionals and ministries throughout the Nordic countries, as well as various gender equality and educational institutions whom have all contributed with knowledge on research, projects, initiatives, etc. in question in supplement to the already published research.

The content of the report is sought quality-assured by collecting comments throughout the writing process - partly from The Nordic Council of Ministers and from The Ministry of Children, Gender Equality, Integration and Social Affairs, Denmark and partly from a number of colleagues positioned in different Nordic countries.

\section{Best practice cases}

As part of the mapping, we have identified number of best practice cases (actions or activities). Throughout the report we describe these cases based on available descriptions and telephone interviews with key play- 
ers. However, it is important to mention that, as part of the mapping, we have not come across that many actions or activities that have had an inherent gender perspective or where the pronounced aim has been to solve some of the challenges that we present in the report. The best practice cases presented in the report were selected on the basis of an assessment that they (either in their "pure" form or elements of them) have cross-Nordic potential for solving a range of the different types of problems that we have come across in the literature on the topic. Hence, the idea is that they subsequently can provide a basis for further discussions of their potential transferability in relation to solving the problems in the Nordic peripheral areas identified through the mapping.

\section{The structure of the report}

The report is thematically divided with the possibility of finding information divided by country within the various themes. Hence, the report is divided in a total of six different themes where, within each theme, a cross Nordic perspective is accentuated. The six themes are:

- Living Conditions, Demographic Transformations and Gender.

- Education, (Im)Mobility and Gender.

- Place Attachment, Everyday life and Gender.

- Gender, Work and Work Life in Transition.

- Changing Gender Relations and Gender Norms.

- Young Men and Masculinity/-ies in Peripheral Areas.

The mapping also draws on perspectives form migration and mobility research, cultural and human geography, research on rural and regional development, innovation and entrepreneurship, as well as, youth and education research. However, the controlling element in the selection of existing research and literature has cf. earlier prevailingly been on contributions from gender research or on other types of contributions with a gendered angle. The mapping primarily focusses on research and literature published after the year of 2000. 



\section{Living Conditions, Demographic Transformations and Gender}

With 3.5 million square kilometres and a population of only 26 million people, the Nordic Region has the lowest population density in the world, which means that large parts of the Nordic countries are uninhabited or has a very low population density. As described in the introduction, this creates various challenges for remote areas, for example in relation to employment, education and infrastructure. At the same time, there are a number of significant demographic and geographic differences between the Nordic countries. In this section, we outline the different characteristics of the living conditions and demographic changes in various remote areas in the Nordic region; characteristics which frame the developments described in the other sections of report. These are essential for understanding of the relationships between gender, education and population flows in remote areas in the Nordic Region. Emphasis in the section is placed on highlighting specific gendered and/or geographic aspects that might influence national development tendencies. In the cases where we have highlighted a particular region in a country, this selection will be accounted for.

\subsection{Sweden}

According to figures from the Nordic Statistical Yearbook 2014, the Swedish population accounts for 9,644,864 million inhabitants; and is thus the largest population among the Nordic countries. The population is, however, very unevenly distributed across the Swedish territory, thus Eriksson et al. write:

If we divide Sweden into two equal halves, 90 percent of the country's population is in the southern half. This uneven population distribution is, historically, a consequence of a scarcity of cultivated land and a large inaccessible mountain range in the North. The territory of Norrland has nearly 1.2 million inhabitants, most of the population living in the coastal areas, which leaves 
large inland areas largely uninhabited, and it is one of the regions that is less favored in global processes of restructuring (Eriksson et al. 2015: 39).

The Swedish population is distributed over an area which covers 447,420 square kilometres, and together with Norway and Finland, Sweden holds a number of large forest areas, which means that forestry traditionally has been a large industry. The vast natural areas also provides Sweden with a unique opportunity to extract energy from their natural resources with the result that, "...in 1960 nearly 90 per cent of the total production of electricity in Sweden stemmed from hydropower" (Haagensen 2014: 34).

In 2013, Sweden experienced the largest net migration among the Nordic countries, and towards 2035 Sweden can expect a population growth of 16 percent. The Nordic countries are frontrunners on a number of equality areas, for example in terms of equal pay. Sweden is particularly proactive for example in terms of their language and their use of gender contracts. It is, therefore, surprising that Sweden has the largest discrepancy between men and women's wages (Haagensen 2014).

Like a number of the other Nordic countries, Sweden is also facing demographic challenges related to the issue of peripheral areas experiencing emigration and changes in living conditions, which in part is due to a decline in traditional industries. At the same time, a number of the peripheral areas, including Norrbotten in northern Sweden (the region borders with Lapland in Finland), experience that young people (and especially women) choose to move away from local areas. Johansson, Stenbacka and Nordfeldt (2005) point out with reference to the yearbook from 2004:

\footnotetext{
It is a widely known phenomenon that areas with poor labour market may experience an excess of men. This is partly because women to a larger extent are moving. This has also occurred in Norrbotten where the number of women per 100 men is below the national average. The strongest imbalance is in Pajala, where there are 68 women per 100 men. A report by the Rural Development Agency shows that it has come to a breaking point where even the men leave the rural areas, which is obviously serious and suggests that the outlook for the northern municipalities is even worse (Johansson, Stenbacka and Nordfeldt in Berglund, et al.: 2005, 35-36, own translation).
}

At the same time, Berglund et al. (2005) point out that it simultaneously looks like women who live in remote areas are better educated than the men who live there, which can be explained by the fact that most of the current occupations are now in the public sector; which has a majority 
of women. This is connected with the notion that traditional male jobs, like forestry, are declining. Berglund et al. explain that:

\begin{abstract}
Developments in employment levels in Norrbotten point towards a general trend of higher employment levels for women than for men in most of the municipalities, which applies to the entire Arctic region. In many municipalities in northern Sweden, the public sector is the largest employer, especially for women. From the 1970s onwards, the number of employed in the forest industry declined dramatically. Since it was the largest employer of men, it increased male unemployment rapidly. Women were not working in the forestry industry, and thus were not affected as a consequence of the crisis in the forest industry (Berglund et al. 2005: 11, own translation).
\end{abstract}

\title{
1.2 Norway
}

The Norwegian population is 5,109,056 inhabitants who are quite unevenly distributed across the country's 323,771 square kilometers (Haagensen 2014: 15). Thus, the population density varies hugely between the different regions. Norway's most sparely populated area is the northern area:

Northern Norway covers more than a third of the total area of Norway; still, less than one out of ten Norwegians lives there. The region consists of three counties, of which Finnmark, the northernmost and easternmost county in Norway, is the largest-larger than all of Denmark by area. Despite its size, it is the least populated area in Norway with approximately 72,000 inhabitants (Eriksson et al. 2015: 37).

The fishing industry is still a large business in Norway, especially in remote areas; the industry employs a large number of people. Norway is rich in natural resources such as oil, which the Norwegian GDP per capita is a clear indicator of, as it is about $80 \%$ higher than the EA17 average. This also means that Norway has a very high standard of living. Additionally, Norway (along with Iceland and Åland) experiences the fastest population growth in the Nordic region. In Norway's case this means that within the next 25 years, the country is expected to have a population increase of $28 \%$ (Haagensen 2014).

In their report, Angell et al. (2013) address the issue of how to develop attractive local community and labour market regions in northern Norway. Angell et al. stress that the northern regions in Norway are very different in terms of economic growth and employment opportunities. The regions have significant challenges in recruiting labour, and the difference between outward migration and relocation is very large. 
Thus, local communities are dependent on immigration, which leads to struggles between regions in the race for attracting residents, and thus employees to their particular region. The northern regions in Norway experienced an employment growth of 7 percent in the period from 2003 to 2011; this is significantly lower than the national average, which is 13 percent (Angell et al. 2013).

In absolute terms, the greatest employment growth has been in health and social services, construction, retail trade, scientific and technical activities and teaching. Marine industries (fishing, fish processing and breeding of new species) is still the sector that is relatively strongest represented in Northern Norway compared with the national average (Angell et al. 2013: 12, own translation).

Angell et al. have not made a separate study of men and women in relation to potential gender differences in the characteristics of an attractive locality in Northern Norway. However, Angell et al. point out, based on their results, that it is essential to conduct more thorough analysis of the gender dimensions. In a number of their analyses they have, nonetheless, distinguished between men and women, and some of their results provide an insight into different gender dimensions and issues. For example, their studies show that a gender discrepancy is detected in relation to accessibility to work, including commuting. It remains the case that there are more who commute out of the Northern Norwegian municipalities than in, although the proportion of commuters who commute to Northern Norway has increased - here it is an important point out that it is mainly men who are commuters:

\footnotetext{
Commuters are in this context largely men, and this fact is important both for the communities they commute to and from. The unemployment rate in the north is declining and is reaching the national average. Among men it is still slightly higher than elsewhere in the country, while the unemployment rate and labour force participation among women is close to the national average. Meanwhile, the regional differences within the region also decreased, although some regions still have higher unemployment, especially among men (Angell et al. 2013: 12, own translation).
}

In line with a number of the other Nordic countries, Norway is experiencing an ageing population. Angell et al. emphasize that especially the age group 20-39 year olds is critical to a region's future growth, and this group in particular has declined in the northern regions in Norway especially with regard to the proportion of women. 
The proportion of people in this age group has declined, and in particular the number of women. At the same time, the number of childbirths has declined. The largest change in the population demographics is the increase of middleaged and elderly people in Northern Norway, and the population pyramid in the north is more "top-heavy" than in Southern Norway (Angell et al. 2013:

12 , own translation).

Due to the specific issues related to Northern Norway in connection with issues of gender, education and population flows, a large part of the remaining sections of the report on Norway will be based on literature that focuses primarily on this area. However, also other Norwegian peripheral areas will be covered (e.g. by reference to Båtevik (2001) whose research is based in the West Country, Rye (2006) who studies Central Norway and Bye (2010) who refers to Trøndelag).

\subsection{Denmark}

Following Sweden, Denmark has the largest population among the Nordic countries. In 2014, 5,627,235 million people $(2,834,956$ million women and 2,792,270 million men) lived in Denmark on an area that is 43,561 square kilometres. Denmark has a much smaller area than some of the other Nordic countries such as Norway, Sweden and Finland. In addition, Denmark (excluding the Faroe Islands and Greenland) has the highest population density (130 persons per $\mathrm{km}^{2}$ ) among the Nordic countries.

Most of Denmark's natural areas are, in contrast to the other Nordic countries, cultivated which means that Demark does not hold the same extent of large uninhabited wilderness areas (Haagensen 2014: 22; 36). Hence, the geographical distances in Denmark can - in relation to a number of the other Nordic countries - seem very small and manageable in terms of mobility. Due to the geographic landscape, not many regions in Denmark can be described as peripheral compared to the other Nordic countries.

The island of Bornholm, located in the middle of the Baltic Sea, can be described as the most peripheral area in Denmark. This means that the island has distinctive challenges related to its geographical location which vary a lot from other parts of Denmark (Dahlström et al. 2006). Isolated in the Baltic Sea, there are limited opportunities to get to the mainland; either by plane or ferry.

Transport to and from the island is both expensive and time demanding and thus, for most people it is not possible to live on Bornholm and to maintain a job that demands a daily presence outside the island. Thus, the labor market 
in Bornholm can be characterized as a closed one, which leads to unoccupied jobs and/or unemployment (Dahlström et al. 2006:32).

The population on Bornholm is declining in contrast to other regions of Denmark (overall). This is mainly due to outmigration among young people who are forced to move from the island if they wish to take an education. Notwithstanding, other parts of Denmark are also experiencing a disproportion between the periphery and the centre. Particularly since 2007, there has been an increased focus on the peripheral areas in Denmark, and the challenges that local populations in these areas are facing (Christensen and Pristed Nielsen 2014).

In the coming years, Denmark will experience a variety of demographic changes. Despite being the most densely populated country in the Nordic region (and holding the second largest population among the Nordic countries), the birth rate in Denmark is among the lowest in the Nordic countries (this is despite the fact that the birth rate in the Nordic region is generally high compared to other non-Nordic countries). Denmark may, at the same time, in the coming decades look forward to a moderate increase in the number of elderly people (i.e. people over 64 years). While Denmark is experiencing an ageing population, the country has also in recent years seen a population growth, which is due to the fact that the number of births exceeds the number of deaths, but also that there is a positive net migration - hence, Denmark is experiencing increasing immigration without a large outmigration. The net migration was highest in Denmark in 2013, and the Danish population is expected to grow by between 7-8 percent until 2035 (Haagensen 2014: 38-40).

Demographic changes in the form of increased population growth, an ageing population and increased urbanization is part of the discourse in Denmark concerning changing living conditions, particularly in peripheral areas. Particularly scholars, professionals and politicians have presented challenges related to these changes in the past few years. This should be viewed in the light that Denmark since the 1990s has seen an increasing centralization of growth. According Aner and Hansen there is a strong consensus in the research community that the growth and development of Denmark takes place, "in a number of major functional regions around the big cities, while places in the periphery of the growth centres experience stagnation or decline in terms of population, functions and economy" (Aner and Hansen 2014: 13, own translation). The centralization of growth contributes to limiting employment opportunities in peripheral areas, often resulting in requirements for a high degree of mobility for those who live or settle in remote areas to find appropriate employment. 
External factors also affect the living conditions in rural areas of Denmark. Thus, the increased competition due to globalization has contributed to traditional industries being challenged. This development is important for the next generation of residents in peripheral areas, as traditional business opportunities are declining. More - especially young people - move to larger cities where career opportunities are better. Hence, a number of peripheral areas in Denmark experience that their population is getting older; a consequence being that the areas will be "shut down" (Danske Regioner 2010).

Outmigration from peripheral areas should be seen in the context of the financial crisis. Aner and Hansen argue in their studies that it is peripheral municipalities that are affected the most by the financial crisis. This means that if you compare 2010 with 2003, the peripheral areas have experienced both a decline in the number of jobs and a decline in the influx of highly educated people from the cities (Aner and Hansen 2014).

Denmark is also facing a number of challenges related to the infrastructure in peripheral areas, as well as issues with creating future sustainable jobs (Danske Regioner 2010). A report by Danske Regioners (Danish Regions) from 2010 Growth in Denmark - Challenges and opportunities for peripheral areas examines the challenges and potentials in peripheral areas in Denmark, and presents a number of recommendations for the different Danish regions. For example, it is emphasized that:

The infrastructure must ensure efficient mobility and thus it should support future transportation needs and business development. Accessability is important for settlement, education and businesses in all of Denmark, and is, therefore, an important part of regional growth strategies (Danske Regioner 2010: 9, own translation).

Besides tangible infrastructure issues, demographic changes are experienced in a variety of ways for those who live in the periphery - especially young people in these areas face the changing living conditions close up. The centralisation of education means that young people at an early point will have to make a decision which directly affects their future job opportunities. Faber et al. point out in the report Unge på Kanten (Youth on the Margins) that a prevalent discourse advocating that young people today have far more opportunities for education than before exists. At the same time, this means that young people at an early point will have to choose between a myriad of competing educational alternatives. Faber et al. highlight that having to choose a secondary education is one of the first complex decisions young people must take in their life planning: "Not just in a here-and-now perspective, but also because it sets a direc- 
tion in relation to a future choice of education and/or profession" (Faber et al. 2014: 21, own translation) - and this may be further complicated by the fact that for some young people, choosing a particular educational direction can mean having to move away from their community.

The choice of secondary education is often an important part in shaping young people's identity and their identity development; thus, changes in living conditions and demographic changes affect young people's development. The limited supply of education in peripheral areas means that young people in the centres often have a better range of educational opportunities in their community, whereas education choices for young people in the periphery often has a greater impact, since it in many cases means that the person will have to leave his/her community.

It is not just young people in peripheral areas who are experiencing challenges related to centralisation. Centralisation of employment opportunities has also brought the need for a greater degree of mobility among those who live in the periphery. Centralisation of employment opportunities should be seen both in the context of globalisation and increasing competition, but at the same time, regional development in the periphery has also been characterized by structural changes in the public sector. Tanvig highlights in her research that:

\footnotetext{
The reduced supply of jobs is not only due to structural changes within agricultural development, which in itself has drawn numerous derivative businesses away from the country districts. It is in particular other professions, which in recent decades have made it possible to make a living in sparsely populated areas, which are now declining. The structural reform that came into force in 2007 has reduced employment in the public sector in these areas - both because of the reduced number of municipalities, and the wave of centralisation which followed (Tanvig 2010:5, own translation).
}

Especially the reduction of employment in the public sector in the periphery has a gender dimension, since it is women in particular who are employed in the public sector. Thus, some women have experienced that their employment opportunities are limited in peripheral areas, which poses increasing demands for mobility, but which also in some cases complicate women's possibilities to continue living in the periphery (Tanvig 2010). 


\subsection{Finland}

Finland covers an area of $338,432 \mathrm{~km}^{2}$ and had per $1^{\text {st }}$ of January 2014 a population of 5,451,270 million inhabitants. Finland has some of the largest forest areas in the Nordic region, and, at the same time, some of the most sparsely populated areas in Europe. The majority of the Finnish population lives in or around the capital of Helsinki in southern Finland. A large region in the northern part of Finland is named Lapland, and this area has the lowest population density in the country. In 2001, the people of Lapland accounted for $3.4 \%$ of the Finish population. Lapland consists of 22 municipalities, five of which have more than 10,000 inhabitants, while three have fewer than 2000. Due to its location and low population density, Lapland faces a number of challenges in terms of gender, education and population flows (Haagensen 2014).

According to Abelsen (2005), in 1991 Finland experienced a sudden decline in exports, which had a massive impact on the country's economic growth. This was mainly due to the fact that a large part of the economy was tied up to eastern export, and with the Soviet Union's collapse the Finnish market lost a major buyer of goods. The subsequent upturn in the Finnish economy was not evenly distributed in the country, but localised around a few growth areas. This has led to large regional differences in Finland. It is especially industrial production linked to telecommunications which has helped generate economic growth - and this has mainly been centralised in southern Finland. This means that the northern parts of Finland to a lesser extent have been part of the economic development (with the exception of Rovaniemi) (Abelsen 2005).

The economic development affects gender relations, education and population flows, as it influences living conditions for the various populations in peripheral areas in Finland, particularly in Northern Finland, in relation to employment. A vast amount of research addresses issues related to the Finnish periphery with a special focus on Lapland. At the same time, Finland is facing a number of demographic challenges over the next 25 years. It is expected that Finland (and Åland) proportionally will have the highest number of elderly people in the Nordic region. Latest figures from Finland (and Åland) shows that in 2030, people over 65 will constitute $50 \%$ of the adult population in Finland. This creates challenges in terms of maintaining a functioning work force, and, at the same time, it requires large amounts of resources for the care sector (Haagensen: 2014).

Lapland takes a special place in the Finnish debate on peripheral areas, population flows and gender, as described above. According to 
Westman (2005), Lapland accounts for $29 \%$ of the total area of Finland, but only $3.4 \%$ of the population resides in this part of the country. Moreover, Westman refers to the notion that Lapland in particular is a peripheral area - both geographically and mentally, "If we think about the geography of Lapland, we can notice that it is a periphery. On the mental level, it is also the place where people live with their own traditions and culture." (Westman 2005: 114-115). The idea that Lapland is a "mental" periphery may be associated with the fact that the labour market in Lapland is largely tied to local resources; thus $27 \%$ of Finland's wild berries are produced in this region, and 36\% of Finland's hydropower is extracted in Lapland.

The notion that Lapland is a periphery with a number of demographic challenges is evident as the population in Lapland is declining, and Lapland is facing a loss of $1 / 3$ of its population in the near future. Hence, there is a growing need for people who can work in the health sector in the region, as the population will age in the coming years (Westman 2005). Due to the specific issues related to Lapland in connection with gender, education and population flows, the majority of the other parts of the report concerning Finland will be based on literature that focuses on Lapland in particular.

\subsection{Iceland}

The population in Iceland is just above 325,000 , however, since the area at the same time is relatively large, Haagensen (2014) states that Iceland has the second lowest population density in the Nordic region, with only 3,6 people per. square kilometres. The population of Iceland - as in many other Nordic countries - has increased substantially during the 20 th century, from around 78,000 in the early 1900 s to nearly 325,000 people today. The increase has been particularly high in the late 20th century, as the population has grown by $28 \%$ in Iceland from 1990 until today. Furthermore, Haagensen points out that the number of childbirths in Iceland is relatively high, which is the reason for the high population growth rather than immigration. For the same reason, population projections points to a growing population in Iceland - the expectation is a $22 \%$ increase within the next 25 years - see Haagensen (2014).

The large population growth in Iceland covers a significant variation in progress in different parts of the country. Thus, the population in the Reykjavik area has increased by a factor of 12 (from about 15,000 to about 180,000 ) in the period 1910 to 2001 , whereas the population in 
the rest of the country has increased by a factor of 1.5 . Overall, this means that the metropolitan area in 2001 accounted for about $63 \%$ of the country's inhabitants, against about 18\% in 1910 (Bjarnason and Thorlindsson 2006: 292). As further described:

The rapidly growing capital area surrounding Reykjavik offers a diversity of professional, service, government, and business opportunities that cannot be matched in other areas of the country [...]. In sharp contrast, occupational opportunities in many rural areas are rather limited and tend to be highly gendered (Bjarnason og Thorlindsson 2006: 292).

Due to the extreme population concentration in Reykjavik, which accounts for $63 \%$ of the population of Iceland, the majority of the other sections on Iceland within the report are based on literature that focuses on all other geographic areas in the country - often with contrasting comments in relation to the capital region, which often serves as a counterpoint to gain insight into trends concerning gender, education and population flows in Iceland's peripheral areas.

Iceland has undergone a number of amalgamations of municipalities from 224 municipalities in 1974 to 79 in 2006 (Styrkársdóttir 2009: 151), and the trend is continuing (Wildy et al. 2014). This is interesting from the perspective put forward in a qualitative study by Edvardsdóttir that,"the smaller the community, the more male dominated it was" (2013: 81). Styrkársdóttir writes about the number of women in local politics in Iceland that, "it is evident that the larger the municipality, the greater is the proportion of women" (Styrkársdóttir 2009: 151). However, overall the development in women's share in Icelandic local politics is low: from 1 to $36 \%$ in the period 1950 to 2006 (Styrkársdóttir 2009: 150).

As in many other sparsely populated areas in the Nordic region, it is especially young women who leave the smaller towns in Iceland (Hamilton and Otterstad, 1998; Karlsdóttir and Ingólfsdóttir 2011). This is largely due to the fact that many of the smaller towns and villages are characterized by an economic structure where the primary and secondary sectors historically have been dominant, which often leaves well educated women few opportunities for achieving an attractive job in the local area (Hamilton and Otterstad 1998: 11). Hamilton and Otterstad included 189 municipalities in Iceland in their 1998 study, and concluded at that time that there was a clear correlation between the population size in a municipality and the number of women relative to men: the smaller a community, the greater a loss of women (Hamilton and Otterstad 1998: 15). Reykjavik, on the other hand, had a small overrepresentation of women. However, it is not the size of the community itself, but rather the nature 
of its labour market (including the number of jobs in the tertiary sector and a diversified supply of jobs) that are critical to whether a municipality is likely to encounter a discrepancy between the number of men and women (Hamilton and Otterstad 1998: 20). The continued relevance of this finding today is found in a recent qualitative study of women's motives to remain in the small town of Husavik on Iceland's north coast (Karlsdóttir and Ingólfsdóttir 2011), as well as a case study (with a less gender specific aim) of the recent labour market developments in the relatively isolated area Eyafjörður (Dahlström et al. 2006).

Skaptadóttir and Wojtynska (2008) emphasize, however, that even the most remote and "isolated" Icelandic fishing communities have always been globally connected and have had many interactions with the surrounding world due to strong traditions of work-related mobility. In Iceland it is also, according Rafnsdóttir and Ómarsdóttir (2010), "common that young people go abroad, either to study or find work. Many continue to live abroad, although the majority return within a few years" (Rafnsdóttir and Ómarsdóttir 2010: 69, own translation). They stress, however, that the same return migration pattern does not apply when you look at outmigration from sparsely populated municipalities to urban areas, and that the peripheral areas that experience the largest outmigration are dominated by traditional male occupations in the primary sector (Rafnsdóttir and Ómarsdóttir 2010: 69-70). They also refer to a master's thesis, pointing to how "a monotonous working life in the local community and better wages in metropolitan areas are primary reasons for migrating among women aged 20-39" (Rafnsdóttir and Ómarsdóttir 2010: 70, own translation; see also Sigurgeirsdóttir and Rafnsdóttir 2009).

Another factor in women and men's living conditions and opportunities in society relates to ideas about the role of families and the responsibility for the family's well-being. Family benefits are income regulated in Iceland, and to a larger extent than in the other Scandinavian countries, the Icelandic welfare system is build on the notion that the extended family carries a responsibility for other family members.

The authorities have, up until present day, assumed that the extended family, particularly women, are caregivers of young and old, and thus partly replace public welfare services, which are common among the Scandinavian countries. This attitude may have contributed to the fact that the welfare system, including the idea of gender equality, has developed at a slower pace in the West Nordic Region than in the other Nordic countries (Rafnsdóttir and Ómarsdóttir 2010: 84, own translation).

This interpretation is to some extent contradicted by Heijstra et al. (2013), as they characterize the Icelandic welfare system as "defamiliar- 
ized". Participants in Rafnsdóttir and Ómarsdóttir's study also expressed a sense of, "embarrassment when applying for social services, which they saw in relation to the emphasis on diligence in Icelandic culture" (Rafnsdóttir and Ómarsdóttir 2010: 81, own translation). Furthermore, Edvardsdóttir (2013: 84) points out that a dominant neoliberal discourse is present in Iceland, which places the responsibility and opportunities in life on the individual. Despite the existence of the formal framework of the welfare system, a number of informal frameworks directly or indirectly affect the individual opportunities for men and women in Icelandic society.

When discussing living conditions in Iceland, it is necessary to mention the economic collapse in Iceland in 2008. In addition to the direct economic consequences of the crisis, Styrkársdóttir states that the crisis also led to a deep distrust of politicians among the Icelandic population, and the 2009 elections marked a sharp division in Icelandic politics, as no less than $43 \%$ of the elected members of the parliament were women, which was a new record (Styrkársdóttir 2009: 132-133).

\subsection{Greenland}

With a population of 56,282 people per January $1^{\text {st }} 2014$, and only 0.14 people per. square kilometres, Greenland is, according Haagensen (2014), the most sparsely populated country in the Nordic region - and also the country with the largest infrastructural challenges. The Greenlandic population has increased nearly fivefold since the beginning of the $20^{\text {th }}$ century, from around 12,000 inhabitants to 56,000 today. Greenland is the only country in the Nordic region with a population that at present moment is expected to decline (Haagensen 2014). Greenland's population consists predominantly of men; statistics from January 2014 show that there are 29,730 men and 26,552 women. At the same time, the overall population is relatively young, as about $33 \%$ are under 20 years. According Weyhe this is, "a very high number compared to the other Nordic countries, where the average figure is about 15 percent" (Weyhe 2011: 252, own translation). It generally applies to the West Nordic Region (Greenland, Faroe Islands and Iceland), that there are more men than women, as more women choose to move from these areas (Rafndóttir 2010: 7). Women's share of the population in Greenland was higher than men's until the latter half of the 1950s (at the start of the modernization era). But since the 1960s, there has been a signifi- 
cant decline in the number of women who live in Greenland (Poppel 2010: 44-45).

The female deficit in Greenland can be related to several factors, including the comprehensive immigration to Greenland from Denmark, especially from the 1950s onwards, mainly consisting of men in the working age, who for a - typically short - period chose to settle in Greenland. Poppel (2010) states that, "Immigration has caused a permanent gender bias with a predominance of men in the working age. It is still predominantly men who move to Greenland, and moreover more women than men emigrate from Greenland" (Poppel 2010: 45, own translation). A much more detailed and precise presentation of the development over time is found in Hamilton and Rasmussen (2010), who, among other things, point to how fluctuations in the ratio of women to men in Greenland has historically been closely linked to developments in parameters such as climate, access to technology and modes of production. This contributes to the overall picture, with $12 \%$ more men than women in Greenland (Poppel 2010: 45; see also Poppel and Kleist 2009: 345). According to Hamilton and Rasmussen, the major part of the "female deficit" in Greenland can be explained by taking into account the Greenlanders living in Denmark, but born in Greenland. Including these in the population count, we go from having 113 men per. 100 women in Greenland, to having 103 men per. 100 women (Hamilton and Rasmussen 2010: 50).

The population of Greenland is, according to a report from Nordregio (2010), geographically highly mobile, and the same report points out that relocating in Greenland is much more frequent than elsewhere in Scandinavia. Previously, Greenland has experienced that more young men than young women relocated, but today the number of men and women, who relocate is fairly similar. However, although the number of men and women who move does not diverge to a large extent, there is a large difference in the way they move, and this difference results in a gradual decline of women in smaller communities:

\footnotetext{
As regards the women, there is a "step-stone mobility pattern" in which they incrementally move from smaller to larger localities, and eventually choose to leave the country, while men on the other hand are more temporary movers, seeking jobs with shorter or longer duration in nearby towns and villages, but also more often return to where they came from (Nordregio 2010: 16, own translation).
}

In Greenland, and generally throughout the Nordic countries, there is a tendency towards progressively greater urbanisation - that is that people are locating to major cities (Hansen, Rasmussen and Weber, 2013). 
Overall, this means that there is, "a net outmigration from the small villages, a rough balance when it comes to cities, and a net outmigration from Greenland as a whole" (Nordregio 2010: 123, own translation). This trend will probably continue, as a survey among the Greenlandic workforce, which among other things examines intentions to move, shows that the number of people who wish to move represents a relatively larger share in the peripheral areas than in the cities. At the same time, the Nordregio report shows that a large number of the people who are planning to move, indicate that they would move along with others, which would sharpen the urbanisation tendency further, since relocations thus would increasingly take shape as collective actions wherein all or part of families choose to move together. Thus, the report points out that, "the need to address mobility patterns from a family perspective more than as individual mobility plans, seems to be increasing." (Nordregio 2010: 129, own translation).

In regards to Greenland, it is particularly evident that there is a significant regional factor in relation to mobility patterns, and most relocations happens between neighbouring towns and small villages (Nordregio 2010). On $1^{\text {st }}$ of January 2008, the former 18 municipalities in Greenland merged into four large municipalities (Poppel and Kleist 2009: 352). There is a huge difference between life in the cities and the small villages in Greenland. Thus, in Greenland there are 67 villages with between 20 and 500 residents. Poppel and Kleist write, "all villages are characterized by a sense of belonging and high dependence on nature through fishing, hunting and sheep farming" (Poppel and Kleist 2009: 354 , own translation). Locally, there are a number of areas where the decision making regarding housing and care for children and young people are placed within the village councils, which have decisionmaking power and jurisdiction on several areas. Poppel and Kleist highlight that generally fewer women than men are represented in the political bodies in Greenland, and women are particularly underrepresented in village councils (Poppel and Kleist 2009: 355).

It is worth noting that although there has been an increase in the number (in absolute terms) of migration to Nuuk and Denmark, respectively, Greenland has experienced a recent relative decline in the number of moves to and from Denmark (Nordregio 2010: 14). The vast majority of respondents in the survey, which provides the basis for the Nordregio 2010 report, states that work (either their own or partner) or education (either own or partner) is the main reason for moving (Nordregio 2010: 14). This pattern is reflected in a much smaller study by Poppel, based on focus group interviews among young women in Nuuk. In this study, the 
women state better educational opportunities, internships and job opportunities as major reasons to move, and in relation to the question of moving out of the country, a reason might be the desire to obtain an education which either does not exist in Greenland, or because they believe that an education abroad is perhaps better than the education available in Greenland. Also, the lack of housing and student accommodation was indicated as a reason to move to Denmark (Poppel 2010).

The report from Nordregio (2010) confirms that there is a larger tendency to migrate among people with an education. The individuals who move and acquire a higher education rarely return to their original place of residence. According Rafndóttir, who in this context is writing generally about the West Nordic Region, different sources have pointing to the fact that, "particularly young women from peripheral areas who move away, perceive it to be difficult to return back home. The labour market is described as male, educational opportunities are limited and women- and familyfriendly welfare offers are few" (Rafndóttir 2007: 9, own translation). In a recent study, Rafndóttir has concluded that women in Greenland to a larger extent move for education and jobs, but also to get access to a better range of public institutions such as hospitals, nurseries and recreational activities (Rafndóttir 2010). Poppel stresses in this regard that it is important to keep in mind that the Greenlandic welfare model is significantly different from for example the Danish one, as a number of social benefits are based on needs assessment (Poppel 2010: 66), which in many cases results in women being more dependent on their families than for example in Denmark.

Nordregio's extensive report Mobility in Greenland from 2010 provides the most thorough analysis of the developments in population flows in Greenland in recent time - the first part of the analysis in the report is based on register data from the period 1995-2008. Particularly in relation to this report, we highlight a number of key points from the register-based analyses concerning mobility and gender, as well as mobility and education. ${ }^{3}$ Regarding mobility and gender, the report highlights that there are differences between Greenlandic men and women's mobility patterns, especially when examining the difference between those who move permanently and those who move temporarily. Thus, more men than women move temporarily, while more women than men move permanently. According to the report, this leads to "a general and

${ }^{3}$ Chapter II e and II $\mathrm{j}$ in the original report. 
growing deficit of women" (Nordregio 2010: 62, own translation). This shortage of women varies from place to place, and "there is a clear correlation between gender balance and economic activities, because more activities in the tertiary sector also means a relatively higher proportion of women" (Nordregio 2010: 62, own translation). In other words, the more developed the service sector is at a given location, the smaller the deficit of women is expected to be. The report explains that, "women more often than men are looking for job opportunities and social conditions which are often better accommodated for in the larger towns, particularly in Nuuk, but especially outside Greenland" (Nordregio 2010: 64, own translation). This also contributes to the mobility pattern describes in the report described as women's "stepping stone mobility", where Nuuk becomes a temporary migration destination for those women who later end up moving out of country altogether.

The report from Nordregio presents in its concluding chapter a variety of future scenarios built on various statistical data. And although the individual projections vary depending on the basis of the calculations, the overall trend for Greenland is clear: there will be an increasing concentration of urban population and the elderly population will account for a growing share - some of the scenarios operate with a 1-1 dependency rate, since for every 100 in the working age there will be 50 older $65+$ and 50 children/adolescents who have not yet entered the labour market (Nordregio 2010: 211-228). Altogether, Greenland therefore faces a number of demographic challenges that are clearly "gendered", and where it seems obvious that any solutions to these problems must necessarily take into account gender perspectives in terms of which living conditions are canvassed and which mobility patterns these preferences results in.

As challenges concerning gender, education and population flows to a large extent concern Greenland as a whole and not only development in the small villages in Greenland, a large part of the research and literature that this report contains concerns Greenland in general.

\subsection{The Faroe Islands}

With a population of 48,228 people by January 2014 spread across an areas of over 1,396 square kilometres, the Faroe Islands are home to the second smallest population in the Nordic countries (the smallest is found on Åland, Haagensen 2014). With a population density of 34.6 per. square kilometres (Haagensen 2014: 10) the Faroe Islands is the 
most densely populated area of the Nordic countries except from Denmark. Similar to the total population across the Nordic region, the population of the Faroe Islands has grown quite dramatically, and has tripled from about 5,000 in 1800 to about 15,000 people in the early 20th century (Gaini 2006), and again tripled to about 48,000 today (Haagensen 2014: 35). The Faroe Islands experiences a relatively high birth rate (in 2013, 260 more children were born compared to the number of deceased), which means that there is a natural population growth. At the same time, however, the islands experience a small net out migration, which means that the total population growth in 2013 was 136 people (Haagensen 2014: 39). In terms of immigration to the Faroe Islands, individuals from the other Nordic countries continue to exceed the number of individuals from non-Nordic countries (Haagensen 2014: 46), although by now there is a small group of non-Nordic immigrants in the Faroe Islands (Høgnesen 2015).

As in the rest of the West Nordic Region, there are more men than women in the Faroe Islands (Knudsen 2010: 13). This is partly due to the fact that more women than men migrate. Giani (2011b) describes the current trend:

\footnotetext{
The Faroese peripheral municipalities, especially small isolated islands, are losing their young people. First the girls move, and then the boys follow. Young men who refuse to move, for example those who are waiting to take over a well-functioning farm, have a hard time finding a spouse. The young people are moving to larger and more central villages or to the capital Torshavn. Many young people, especially those who are planning to take a higher education, move abroad. Not surprisingly, Denmark is the most popular destination on the European continent. And girls are currently the most ambitious and daring in relation to realizing their dreams of a high education, a stimulating career and an exciting lifestyle (Gaini 2011b: 3, own translation).
}

The Faroe Islands consists of a total of 18 large and small islands, and infrastructure and geographical conditions are therefore of great importance for local development and for people's everyday lives. The Faroe Islands is divided into many, often very small municipalities - since the last amalgamation of municipalities in 2009, there have been 30 municipalities with large variation in the number of inhabitants, "Figures from 2009 (Hagstova Føroya) show that the smallest of them (Fugloy) had 41 inhabitants and the largest 19,675 (Tórshavn)" (Knudsen and Niskanen Nyberg 2009: 330, own translation). In the 1950-60s, the development of the infrastructure took speed, and regular air travel out of the country was established in the 1960s. During the same period, the islands experienced, "a huge population flow from the small villages to 
Torshavn. During those years, the small villages turned more or less into peripheral areas while Tórshavn increasingly assumed the role as the centre "(Jacobsen and Jákupsstova 2005: 186). Hovgaard and Kristiansen (2008) argue that geography traditionally has created boundaries for the local population's mobility outside their own village, but also that the construction of infrastructure such as roads, tunnels and bridges has helped to create "network villages","Today you can live in one place, work in another place, buy commodities in a third, pursue leisure activities in a fourth and go to church in a fifth" (Hovgaard and Kristiansen 2008: 66) - A fact which creates problems for those municipalities which either have not kept up with development or are located outside these infrastructural networks.

Hovgaard and Kristiansen point in particular to three factors (but stress that these are not the only factors) which affect the individual municipality's ability to maintain its population, these are: child care, mobility, and a sense of belonging. Regarding child care, Hovgaard and Kristiansen increasingly see this as a competitive factor for the individual municipality - without good schools and preschools the villages lose their attractiveness to their citizens. It is also essential for the individual citizen to have access to transportation - either in the form of ownership or at least the possibility to access a car. Everyday activities such as shopping and leisure are increasingly separated (Hovgaard and Kristiansen 2008: 68), and hence mobility vs. immobility become a crucial social dividing line. Finally, they argue that sense of belonging continues to play a major role in the Faroe Islands, and that many actually settle down either in their childhood home, or at least in their hometown. As they write, "There is a huge difference between "not being able to leave" and "being able to leave but wanting to stay" [...] Because of increased (auto)mobility, living in the country is now merely a spatially different way of living in a suburb" (Hovgaard and Kristiansen 2008: 69).

Regarding the relationship between geography and gender, Knudsen highlights - in contrast to the situation in Greenland where many small villages are without female representation - that "the number of local governments without female members declined dramatically after 2000" (Knudsen 2009: 330, citing Jacobsen and Jákupsstova 2005: 181, own translation). This does not, however, outweigh the fact that the proportion of women in elected bodies in the Faroe Islands is quite low. At the same time, Knudsen concludes that women seem to play a greater role in local governments in regards to implementation of decisions, rather than in the political decision making process (Knudsen 2009: 333). Gaini writes about geography, inequality and gender that, "the 
boys in peripheral areas have, in principle, the same opportunities and rights as all other people in the Faroe Islands, but they are often less mobile and more entrenched in traditional roles than the girls from the same community" (Gaini 2011b: 4, own translation). At the same time, Gaini highlights that the media tends to present boys from the villages in a stereotypical way; "as provincial and straightforward, not fit for the imagined lifestyle of urban socialities" (Gaini 2011a: 178).

Knudsen points out that although the deficit of women is generally considered a social problem, moving away from the Faroe Islands can from the individual woman's point of view be seen as a solution (Knudsen 2010). The imbalance in the number of men and women in the Faroe Islands is not new (Knudsen 2010: 19), but it is clear that particularly in the crisis years in the 1990s there was a great outmigration from the Faroe Islands, and during this period, it was particularly men who moved away. According to Jacobsen and Jákupsstova more than 7,000 people left the Faroe Islands in the period 1989-1995. They describe the situation as follows:

\footnotetext{
For the first time since the first census in 1801, the population number declined. Emigration meant a new situation, namely that: Now it was not just young people who left, and male breadwinners who sought employment abroad for a shorter or longer period. Now whole families relocated because both spouses sought alternative labour markets. The family economy and the labour market had changed. The families had become dependent on two incomes (Jacobsen and Jákupsstova 2005: 172, own translation).
}

In other words, it is a relatively new development that it is especially women who emigrate. At the same time, there are also more men than women who move to the Faroe Islands (Knudsen 2010: 20). The emigration which occurred in the 1990s is the most significant explanation for the skewed population pyramid that exists today. Only about $1 / 3$ have "returned" and this bias will, therefore, affect society for a very long time to come, especially as it has led to a shortage of women in the childbearing age.

Based on an interview survey among 26 Faroese women, Knudsen attempts to find some explanations as to why so many women choose to move. She highlights the following:

As a whole, they emphasize that the economic conditions and the possibility of getting an education are better in Denmark. In this connection, they refer to the attractive welfare schemes, more choices and care facilities for children. Especially the group of single mother's indicate that economic conditions for single parents are very poor in the Faroe Islands. [...] The women who do not have children emphasize that the Faroese society is more 
adapted to nuclear family and not to different family models and single people. [...] Several of the women point out that the Faroese society is more attractive to men than to women. Men have more choices in terms of both work and leisure. They emphasize that unskilled men are also extremely well-paid (Knudsen in Rafnsdóttir 2010: 21, own translation).

In her proposal for a new PhD research project, Høgnesen (2015, unpublished) states that in conjunction with the current concern about young women's emigration from the Faroe Islands, it should be further explored how non-Western women, who actually immigrant to the country, are doing. She explains:

Like several other places in the North Atlantic region, the Faroe Islands have experienced a shift in the global pattern of migration. The recent 15-20 years has brought a new and rapidly increasing phenomenon to the islands, which is female marriage migration from Asia [...]. While new population groups are thus entering the Faroe Islands, young people - and in particular young women - are leaving the islands, often in pursuit of education, and frequently without returning. Hence, the net out-migration of women from the Faroes Islands has implied a female deficit of 1,079 women in the age of 20-39 as of January 1 st 2014 . As of 1 st of January 2014 the number of women, 20 years and older, born in the Philippines and living in the Faroes Islands, was 79; the corresponding number for Thai women was 72 (Høgnesen 2015, unpublished PhD proposal).

Høgnesen justifies her research interest in this group of "marriage migrants" with the argument that these women may occupy important roles in Faroese society - as spouses to local men, who might otherwise remain unmarried due to a shortage of women, and as mother's to children who then may contribute to the continued development of the Faroe Islands. Høgnesen highlights that it is important to look not only at the demographic changes that the Faroe Islands are undergoing due to emigration, urbanisation and an ageing population, but also to look towards immigrants - and in this connection the group of so-called marriage migrants represents a special phenomenon.

As challenges concerning gender, education and population flows largely concerns the Faroe Islands as a whole, and not only the development in the small villages, sections on the Faroe Islands within the report address the challenges to the country as a whole. 


\subsection{The Åland Islands}

With a population of just 28,666 people and a territory of only 1,580 square kilometres spread over 6,757 islands (Haagensen 2014: 13), Åland's geography is extreme on several parameters. Just over 60 of these islands are inhabited all year round. As described in Dahlström et al. (2006: 89), "Åland is, in other words, small and spatially diffused. This also applies to the administrative structure. Åland has 16 municipalities, of which six are situated in the "archipelago region" (skærgården), which is not connected to the main island by road". Transportation among the islands is reliant on ferry connections, and from the most remote island that is inhabited all year round, the transportation to the main city of Mariehamn is about 5 hours travel with 3 different ferries (Dahlström et al. 2006: 89). There are daily ferry connections to Turku, Helsinki, Stockholm and Tallinn, and also daily flights to the same cities with the exception of Tallinn (Dahlström et al. 2006: 89).

Approximately $40 \%$ of the population resides in Mariehamn, and a further $50 \%$ in the rest of the main island - with the option to commute by car to Mariehamn on a daily basis for work. Only $10 \%$ of the population is spread over the remaining inhabited islands (Dahlström et al. 2006: 90). In 2005, the municipal size ranged from 127 (Sottunga) to 10,780 (Mariehamn) inhabitants (Dahlström et al. 2006: 90). Hovgaard et al. (2004) provide an overview of the size of the 16 municipalities in Åland, emphasizing that the majority of these are quite small. Thus, they report - based on figures from 2004 - that half of the 16 municipalities had between 250 and 999 inhabitants, one was even smaller and only one - namely Mariehamn - had more than 10,000 inhabitants. Dahlstrom et al. describe the very uneven development in individual parts of Åland as follows:

In recent years then the real winners, in terms of the relative as well as the absolute size of the municipal population, have been those municipalities in the vicinity of Mariehamn. The three municipalities closest to Mariehamn increased their population by some $80 \%$ from 1970 to 2004 . As all of these municipalities are directly connected to the expansive labour market in the economic and administrative centre of Åland, their growth is in reality connected to the growth of Mariehamn (Dahlström et al. 2006: 90).

The rapid urbanisation, which has influenced Åland since the 1950s, affects the population distribution on the islands. The average population density was 17.2 inhabitants per $\mathrm{km}^{2}$ in 2004 , but only 4.5 per $\mathrm{km}^{2}$ in the Archipelago not connected to the main island by bridges (Hovgaard et al. 2004: 25). Despite an overall population increase, the population in the 
Archipelago has decreased steadily,"At the end of 2001, the lowest level yet was reached, with a population of only 2.318 people in the archipelago. By contrast, over 5.800 people lived in these municipalities at the beginning of the twentieth century" (Hovgaard et al. 2004: 26). Mariehamn, on the other hand, has grown from 1,090 inhabitants in 1910 to 10,000 today (Hovgaard et al. 2004: 26).

Its geography notwithstanding, Åland experiences a population growth rate that exceeds any of the other Nordic countries - the population has increased 18\% in the period 1990-2014 (Haagensen 2014:36; see also Rauhut et al. 2008: 104), and the increase in population is expected to continue, with an estimated population growth of $25 \%$ over the next 25 years (Haagensen 2014: 40). As Rauhut et al. however underline, concerning Åland's somewhat surprisingly high increase rate,"even modest absolute population increases will show as a large relative population increases" (2008: 115). In the period 1990-2014, the balance between the number of men and women in Åland has almost evened out (Haagensen 2014: 36). On the other hand, it is expected that the dependency ratio will rise dramatically in Åland in the near future, and the population projections indicate that in 2030, $50 \%$ of the adult population on the islands will be over 65 years (Haagensen 2014: 38). As Rauhut et al. describe it, Åland is "greying fast" (2008: 104). Dahlström et al. state that there is a preponderance of young people who move from the islands and a preponderance of older people, who immigrate to the islands (2006: 90).

According Dahlström et al. (2006: 91), Åland has proven fully able to exploit its geographical position in the Baltic Sea by expanding its shipping sector - and as a result, the islands have a higher GDP than Finland.

Best Practice Case: Suomen opiskelija-asunnot, Finland

Suomen opiskelija-asunnot (SOA) is a national interest organisation for student accommodation in Finland. SOA's aim is to represent common interests among student housing associations across Finland, as well as working for the goal of being able to provide student accommodation at reasonable prices. Hence, SOA itself does not own student accommodation, but represents the interests of organisations that do.

One of the members of SOA is "Student Village Foundation of Turku" (Åbo). According to one of the managers at Student Village Foundation of Turku, the students are highly determined to live downtown, and student accommodation placed a mere 5-6 kilometers from the univer- 
sity and the city centre are not particularly popular. Hence, the organisation does not have student accommodation in peripheral areas, but is focusing on larger cities, where demand for student accommodation is greatest.

Despite the fact that the organization does not offer housing in peripheral areas, they have other means of focusing on problems related to peripheral areas in Finland. The student housing organization thus has special attention to students who come from remote areas in Finland, such as Lapland. In practice, this means that when new students apply for a residence, they are divided into a points system. Applicants from peripheral areas, such as Lapland, are awarded more points than students from more densely populated areas, and thus they stand better chances of getting into the student accommodation (though there are no specific quotas for students who come from peripheral areas in Finland). This points classification testifies to student housing organizations which are aware of the problems in relation to young people who come from the Finnish peripheral areas, and take responsibility in solving some of the challenges these young people from remote areas in Finland may face in the process of moving from local municipalities in remote areas to take an education. In relation to the gender dimension, the distribution of students living in student residences is about equal in terms of girls and boys, and the organization has not set gender quotas.

Another offer students in Finland can make use of in their studies, is student housing allowances (Bostadstillägget). This scheme ensures that persons undertaking studies in Finland are able to get housing allowances which serve towards paying their rent. This offer can be particularly helpful for students who must move far away to obtain an education and thus are compelled to also leave home in connection with their studies.

Read more at:

- http://www.soa.fi/

- http://www.kela.fi/web/sv/studiestod_bostadstillagg

Best Practice Case: The Special Initiatives Zone - Norway

Tiltakssonen i Finnmark og Nord-Troms ("The Special Initiatives Zone in Finnmark and Nord-Troms") is a long-term strategy to make remote areas in Northern Norway attractive. The goal is to get more people to live and work in peripheral areas, and at the same time turn it into an area where businesses see opportunities for development. The project 
was established in 1990 as an answer to the many crises in the region especially for the fishing industry. "Tiltagssonen" remains a regional strategy that is acted upon, and through which large amounts of resources are being channelled with the purpose of stimulating a positive development in remote areas with the intention to create good conditions for the residents and businesses. Current initiatives include:

- exemption from payroll tax

- lower personal tax

- exemption from tax on electricity consumption

- student debt relief/cancellation

- higher child benefit

- wage subsidies for preschool teachers.

"Tiltakssonen" and the various initiatives that the region has made in this connection, has attracted great interest and a number of analyses and reports have been made regarding the measure, for example the report Tiltakssonen of Finnmark and North Troms - utviklingstrekk and gjennomgang av virkemidlene from 2012 by Angell et al.

It is still the case that during a period of over 20 years, changes in employment patterns will result in emigration from peripheral areas. However, the initiatives within the region have been successful in a number of respects, and demographic trends within the area are slightly better than in other peripheral regions. Meanwhile, employment increased strongly since 2005. From a gender perspective, the initiative has succeeded in attracting and retaining highly educated workers, especially highly educated women, who are employed in the region. This is because the region has a large public sector which traditionally employs many women. However, highly educated men are seen to increasingly move from the region.

Read more at:

- https://www.regjeringen.no/nb/dokumenter/tiltakssonen-forfinnmark-og-nord-troms/id682034/ 



\section{Education, (Im)Mobility and Gender}

In this section, we focus on young people and their educational patterns in the Nordic peripheral areas - including educational choices, mobility patterns, mobility motives, and transport. At the same time, it is necessary to keep in mind that within discussions on education and gender, attention has in recent years been focused on boys' performances in the educational system, and terms such as "the failing boys" has been in used. Often, the differences between boys' and girls' educational performances have been explained by biological differences, including notions such as the idea that boys "lag behind" because they find it harder to sit still and concentrate. Thus, the school system today is considered unable to cope with boys and their needs. Or you could, as we do, emphasize that biology perhaps can explain some differences, but not all, since not all boys perform less well than girls - and also that gender interacts with other social categories, such as, class, ethnicity, locality etc.

\subsection{Sweden}

Trondman (1995) has described how young people in peripheral areas are captured in a vacuum, where future opportunities exist outside the local milieu. He further concludes that the most vulnerable people in peripheral areas are young men with a working class background, as they are unequipped to handle the conditions on the new labour market. Trondman writes:

Their (the young men's) opportunity to escape the worst aspects of the unknown is to stay and maintain the security they find in knowing their environment, their friends and who they are. A confirmation that provides security for the moment, but which will also likely place you on the losing team in the future, is valued in the small peripheral societies (Trondman, 1995: 185, own translation).

Waara (2003) concludes that in the Swedish peripheral areas, young middle class women are the ones who are most likely to move. He be- 
lieves this is linked to the fact that their highly educated mother's encourage their daughters, in particular, to leave the sparsely populated areas. Waara points out that there are indicators that the mother's own difficulties in putting their skills into play in the more restricted labour market in the peripheral areas lead to frustration, which they transmit to their daughters.

Svensson (2006) concludes in line with both Trondman and Waara that there are differences between young people in the Swedish peripheral areas, and that these differences are closely linked to social background. She argues that the middle-class boys who are part of her study from Söderhamn have a clear perception that they are forced to move in order to get the jobs or education they desire. The same goes for the girls from the middle class. Svensson concludes that young people, both men and women - from working class homes, mostly want to stay in the local area; not because they believe that the area offers particularly bright prospects, but because they believe that they have better opportunities to find a way to earn a living here than in other places, and also because this is where they have their family and other networks, and they are familiar with the local labour market.

Gustafsson and Öhrn (2012) conclude in Gender, Achievement and Place: Boys and Masculinities in a Rural Area that among young men in the periphery there is a tendency not to prioritize school life, because it is considered unmarketable in relation to their local labour market. They write:

\footnotetext{
The boys make a distinction between "school knowledge" and "real knowledge", with the latter being the central one. "Real knowledge" refers to capacities of value in "real life", and "real life" is typically seen as equivalent to "outside school" [...] The emphasis on local work for boys and by boys in this study, indicates a local / regional masculinity tied to hunting, crafts, fishing, etc. This might be seen as relating to historically well-known masculinities, which has been under threat for decades (Gustafsson and Öhrn 2012: 2; 5).
}

\subsection{Norway}

According to Paulgaard (2002; 2006; 2012; 2015), young people who grow up in peripheral areas (characterized by decline or stagnation) live under completely different conditions than young people who grow up in places that are characterized by growth and progression. Based on a study from Northern Norway Paulgaard writes: 
In Norway, there have been significant regional differences in young people's educational careers. The education level has been lower among the population in rural and coastal places in the north than in other areas. A substantial proportion of young people (here) experience great challenges in establishing a future in the northern area. Their daily lives seem to exist outside of the visions and optimistic statements of the policymakers. Many of them are young, unemployed men living in small rural places in the northern part of Norway [...] Out-migration is a solution for some, but not for all (Paulgaard 2015: 210; 207).

Paulgaard describes how it, in some Norwegian peripheral areas particularly along the coast, is the norm for young people and even children to work alongside their parents. This tradition means that the school to some extent is seen as a "foreign" element, and as a "time bandit", "Schooling has also been described as a "time bandit", gradually snatching children and young people away from traditional local employment and the socialisation provided by work" (Paulgaard 2002: 99) - and Paulgaard further writes:

Recruitment into this world of work (within the local labour market) has taken place within patterns of informal education for generations. Resistance to school and formal education has been characterised as an important element of culture in the rural north. Such resistance has particularly been strong among young boys (Paulgaard 2015: 210).

Paulgaard states that for many young people in the periphery, pursuing further education after elementary school require that they leave home. Being forced to leave home and their local environment at the age of just 15,16 or 17 years and take care of themselves requires a special discipline and motivation. This precise point is important from a gender perspective, since it underlines that not all young people have equal opportunities. Paulgaard emphasizes that, "Structural divides within the educational provision implies individual challenges, demonstrating how access to higher education is not equally distributed" (Paulgaard 2015: 210) This is probably also why the dropout rate for secondary education is higher among young people - especially young men - from peripheral areas compared to other parts of Norway, "Even when controlling for variables like grades from primary school and social background, geography seems to have an independent effect on the dropout rates" (Paulgaard 2015: 211).

Heggen (2002) has conducted research on young people and educational patterns in Norway and argues that there is a clear gender dimension to this. The data presented in Heggen's article Utkantjentene sin stille revolusjon (the quiet revolution by the girls from the peripheries) dates back to 1999, and the figures are, therefore, of an older date. How- 
ever, a number of Heggen's points are backed by recent research by for instance Paulgaard, Båtevig and Bæck. Heggen highlights, in line with a number of other researchers cf. the previously mentioned, that it is especially young women who have taken a big leap in terms of their educational level. Because of this, women are in many ways front-runners in terms of education. According to Heggen, this development has been particularly evident since 2000 . A break with traditional gender patterns is taking place, and it is changing urban boys' traditional superiority within higher education. Thus, today young women, including women from peripheral areas who have moved to major cities, dominate the higher education programs. This creates new challenges in terms of a new imbalance in young people's choice of education and employment opportunities.

\subsection{Denmark}

Gender researcher Bjerring is in line with the previous conclusion; namely that young women in peripheral areas are more mobile and also more oriented towards pursuing an education than young men in the periphery. Bjerring bases her conclusion on a study she conducted on young people in Skagen (the most northern part of Denmark). Bjerring argues that the difference among young people from Skagen is partly due to the notion that especially girls are critical of leading the same life as their mothers. Both in terms of their career, which is why they strive towards new and different career paths (which often means leaving their local environment), but also because some of them see their mother's as "hinterland women" leading a life they do not want to replicate. Bjerring highlights that the girls' profound interest in education and their willingness to move in order to pursue a higher education should be seen in the context of the gender equality debate in Denmark, and the girls' desire to free themselves from traditional gender roles both privately, but also in relation to the labour market (Bjerring 2000 and 2007).

In 2014, Centre for Regional and Tourism Research in Denmark published a report (Hedetoft and Stefaniak 2014) concerning education with a special focus on peripheral municipalities. ${ }^{4}$ The report highlights that

4 The report is based on data from the 2000 year group of young people. This means all young people in Denmark, who in 2000 was respectively 16,17 and 18 years old. The group has been followed until 2011 and it is this data that the report uses. 
young people's choices are influenced by local occupational structures, and the proportion of young people choosing vocational training is higher in peripheral areas. Despite this, "the situation is, paradoxically, so that it is simultaneously these municipalities that first (and to the largest extent) will be short on skilled labour in the coming years. This development is occurring because a large proportion of young people (also those with a vocational training certificate) from the peripheral municipalities migrate to the cities" (Hedetoft and Stefaniak 2014, own translation). The report further concludes that young people's mobility patterns are closely related to their educational choices, and that it is largely girls who choose a highschool education and later a higher education, while boys more often remain in the local area. Hedetoft and Stefaniak write:

Between 45 and $55 \%$ of men in the peripheral municipalities still live in the same municipality at the age of 28 years. Depending on the municipality, the number of men who remain is 4 to 5 percentage points higher than for women. This is to some extent due to the fact that there are better local employment opportunities within the traditional male-dominated sectors (agriculture, industry and construction sectors), and it is still the case that more men than women opt for vocational training (Hedetoft and Stefaniak 2014: 5, own translation).

Yndigegn (2003), Faber et al. (2014), Hansen (2011) and Hermansen (2011) emphasize mobility as an important parameter in educational choices. Despite the geographical distances in Denmark being shorter than in many other places in the Nordic countries, there are still young people in Denmark for whom educational institutions are located far from their local area, which means that they have to spend a considerable amount of time on transportation. Hence, geographical location does in certain cases also impact young people's educational choices.

Faber et al. (2014) conclude in their report Unge på Kanten (Youth on the Margins), which is based on data collection among young people in North Denmark, that educational supplies for young people living in this particular peripheral area are not always as varied compared to the possibilities for young people living in the centres. Moreover, employment opportunities are often very limited. Geographical inequality has been reinforced in recent years as a result of centralisation of educational institutions and the economic crisis, which has caused a further decline in jobs and internship opportunities, especially in the peripheries. From a gender perspective, it is interesting that the study finds that young men in the periphery have a different - more goal-oriented - approach to education than young women. Although a large number of young people form both groups considers education to be the path to a successful 
life, there is still gender differences in the types of education young people aim at, and the vision they have for their education. While young women see education as the primary route to employment, young men seem to place greater weight on experience in terms of landing a job offer. The boys do not to the same extent as young women find that formal education is needed to get a job - and a number of young men believe that they can learn more outside school than inside the school. Additionally, the report concludes that young women from peripheral areas are more willing to move, which is partly due to the fact that they increasingly seem to feel a "pressure" to move as employment opportunities in peripheral areas are more geared towards traditional male jobs. By extension, the study shows that especially young men who feel attached to their local area are less likely to move.

The project further confirmed that long commutes to and from educational institutions is an important factor for young people; the results showed that a long commute affects young people's mental well-being. Overall, the results indicate that boys commute over longer distances and spend more time commuting than girls. This is the case even when taking into account the unequal distribution of gender found in the different types of educational programs. In this connection, it also plays a role that high-school education is available several places in the region, whereas young people who wish to take vocational training have fewer options. This means that many of the young people in vocational training - especially boys - have to commute relatively long distances. At the same time, it is worth taking into account that the limited educational options in some areas reduce young people's feedom of choice. The result may be that young people enrol in an educational program, which they are unmotivated to complete or they move away from home at an early age to take part in an educational program - both factors which may increase the dropout rates (Faber et al. 2014).

Hermansen (2011) describes in his research on young men in one of Denmark's peripheral areas, Horne county, that young people in this area have the opportunity to attend a local school through $6^{\text {th }}$ grade, and that they then have to commute to Faaborg to take the final years to complete primary school. Faaborg is located about $5 \mathrm{~km}$. from Horne town. Faaborg also has a high-school which makes it possible for young people in Horne to live at home and attend high-school in Faaborg. However, the young people in Horne will have to move from their local area, if they want a higher education, as the nearest university is located in Odense. Thus, they will at some point be forced to make a decision about 
whether they want to move away to get an education, or whether they want to find work in the local area.

Bornholm is another example of an area in Denmark where young people are forced to move if they want an education:

Young people on Bornholm follow the general pattern and increasingly undertake a period of higher education in their early adult lives. This means that the majority of young people have to leave Bornholm to study. Afterwards it is often impossible to find a job on Bornholm, as the demand for highly qualified people is limited. (Dahlström et al. 2006: 27).

A report from Danske Regioner (Danish Regions) states that a local educational environment is a precondition for educational attainment, and also a precondition for growth and development in peripheral areas. One of the recommendations from Danske Regioner is, therefore, to utilise various teaching methods to ensure that young people who live in peripheral areas have access to various types of educational programs, and also to focus on ensuring a geographically diverse range of educations:

\footnotetext{
In an effort to create strong education facilities which simultaneously are located in different geographical areas, the options for displaced education, cooperation between various institutions and IT-supported teaching should be utilised [...] Black spots on the map must be avoided. Young people must be able to attend secondary education within a reasonable geographic distance. Distance affects educational choices and drop-out rates. Therefore, ensuring a geographically dispersed and diverse range of all upper secondary schools and vocational schools is essential (Danske Regioner 2010: 9, own translation).
}

Danske Regioner highlights that it is important to look at further education and retraining, and also that these offers should be coordinated in cooperation with regional councils and regional development fora.

During the past year (2014/15), discussions about the supply of programmes in higher education institutions have been taking place in Denmark. This has been prompted by the government's proposal to downsize some subjects in higher education; the plan has been criticized for being geographically skewed - because universities in peripheral areas seem to be hit harder than other universities - and for producing a gender-biased cut, since the downsizing predominantly affects the humanities, which has a predominance of female students. The downsizing in the number of enrolled students has particularly been motivated by a desire to politically push more students to apply for natural sciences. However, critics argue that the plan will more likely result in fewer women being able to pursue the education they desire. 


\subsection{Finland}

According to Keskitalo-Foley (2013), who conducts research on education and migration in Finland, education is a main focus area in the country, and an area where a large amount of resources are allocated. In Finland, education is seen as an important element in an individual's life and part of forming an identity. At the same time, Keskitalo-Foley argues that there is a widespread perception in Finland that education prevents social marginalisation, as well as being an instrumental part in an individual's development and in achieving social and economic status in society. Keskitalo-Foley emphasizes that from a national economic perspective, education in Finland is regarded as a valuable long-term investment. The focus on development and allocation of resources to the educational sector has from a Finnish perspective also meant that Finnish school children for many years have scored high in international tests, such as, the PISA test. According to Keskitalo-Foley this particular test is seen as a factor for predicting the increase in the Finnish GDP (Keskitalo-Foley 2013).

Figures from the Nordic Statistical Yearbook 2014 show that Finland in relative terms has the largest number of college students among the Nordic countries. Among the 20-39 year olds, almost 19\% of young people are enrolled in a higher education. The proportion of university students in Finland who study science, informatics or engineering is higher than in any of the other Nordic countries. Moreover, along with the Danish youth, young people in Finland are among those in the Nordic Region who receive the largest financial support (Haagensen 2014).

As is the case in many of the other Nordic countries, young women in Finland make up the largest proportion of students at higher education. The large number of highly educated women in Finland is often pointed to as an indicator of women's high status in society - an argument used to present Finland as a country with equality between Finnish men and women. As a counter-argument, Keskitalo-Foley (2013) points out that the high educational level among Finnish women does not influence their position in the labour market. Women do not to the same degree as men benefit from their educational qualifications, which is also the case in terms of wages, employment forms, influence and status in companies. Keskitalo-Foley refers to unemployment statistics from for example Lapland, which shows that unemployment among highly educated men in 2008 was around $11.3 \%$, whereas for women in the same age group, the number was 19.9\% (Keskitalo-Foley 2013: 130-131). 
Finland's vast geographical area means that the country has some of the largest natural areas in the Nordic region, and places where the population density is very low. This has influenced school structure in Finland. The country has a school structure with many small schools scattered around the country. The aim has been to create cohesion between the schools and local communities in the more peripheral regions of Finland, and to allow people to settle in peripheral areas instead of moving to more urban areas. Kalaoja and Pietarinen (2009) writes:

\footnotetext{
From the very beginning (since 1921), the Finnish compulsory education system and the school network have been developed on the principle that the schools must be located close to the pupils (the distance from home to school being less than $5 \mathrm{~km}$ ). In rural areas this has meant that almost every village has had its own school. Due to the reduced birth rate, changes in the economic structure of the countryside, migration and improvement of rural road conditions, the closing of small rural schools started to increase significantly in the late 1960s. The position of small rural primary schools improved only for a while at the end of the 1970s when the current comprehensive school system was founded (Kalaoja and Pietarinen 2009: 109).
}

In the Finnish debate on the closing of small local schools, focus has mainly been on the importance of schools in local communities. At the same time, the number of teachers and recruitment has been discussed and problematized; including the tendency that an increasing number of Finnish teachers prefer to live in more densely populated areas and commute to and from work in peripheral areas. Kalaoja and Pietarinen (2009) explains:

\footnotetext{
...the teacher's role is central to developing a locally oriented curriculum and helping the whole school to become the social centre of the village. [...] Teachers have traditionally lived in the surrounding community or even the school building has been their home. They have themselves been one of the villagers. Several studies have shown that there is an increasing trend for teachers to live in population centres and only work at the village school. Villagers however, consider that teachers living outside the community are not connected to the culture of the village [...] This trend of teachers only being loosely connected to the local community, is a concrete change in traditional village culture (Kalaoja and Pietarinen 2009: 111).
}

In Kalaoja and Pietarinens (2009) work on the educational system and peripheral areas, focus is not on gender relations and a sense of belonging. This could, however, be an interesting angle to add to the analysis of issues regarding the small schools and migration from the Finnish peripheral areas to the centres to pursue an education; discussions which are found in for example Norway and Sweden. 


\subsection{Iceland}

Rennie et al. write in an article on the development of a remote learning project in Iceland, that the fundamental principle in the Icelandic educational system is that, "everyone should have equal opportunities to acquire an education, irrespective of economic status, residential location, gender, religion, handicap, and cultural or social background" (Rennie et al. 2011: 1, with reference to the Ministry of Education, Research and Culture in Iceland, 2005) They note, however, that the equal access to education is lagging outside Reykjavik, as"(m)ost study programmes offered at the university level are only available in the capital area as face-to-face study, but some distance provision is delivered" (Rennie et al. 2011: 2). The range of remote learning programs, however, is booming in Iceland (Rennie et al. 2011: 3).

In a comparative study on the conditions in small rural schools in Iceland and Western Australia, Wildy et al. (2014) find that "a culture of acceptance, where expectations for performance and behaviour are known and shared and not questioned" exists (2014: 104). They point out that this school culture does not necessarily in itself entail a problem, as the children in these schools achieve a good and solid education. Nevertheless, they point out that this "culture of acceptance" may keep the children (and their teachers and the local communities collectively) from more exploratory approaches to teaching, which might for example question traditions and norms. Although Wildy et al. do not address the question directly, one might argue that the "culture of inquiry" which they advocate might also be able to challenge a larger number of boys to pursue an education rather than focus on employment in the local labour market, and also question conventional gender roles and standards.

The predominant "culture of acceptance", which Wildy et al. document, relates to the very small rural schools - and there are relatively many of these in Iceland. "In Iceland around one-third of schools can be classified as "fámennir skólar" or schools with fewer than 120 students" (Wildy et al. 2014: 105). The present understanding of a "school" is a relatively new phenomenon in Iceland - thus compulsory schooling was first introduced in 1936, but even up until the 1960s it was common for children in the countryside to stay at home and help the family during busy periods. According to the data in Wildy et al.'s work, this affects the attitude towards education among the population in peripheral parts of Iceland even today. On the other hand, there has been a strong tradition of home schooling in Iceland (Wildy et al. 2014: 111). In the current context of dropping birth-rates in the small communities, and with the 
amalgamation of municipalities, it has been argued that the small schools' futures are uncertain; an issue which was also depicted in relation to the small schools in remote areas in Finland.

Besides the issue of equal access to education regardless of residence, it is also important to ask the question of whether boys/men and girls/women have equal access to obtain the education they desire. Among the Nordic countries, Iceland has the second highest percentage of university students (the highest number of university students is found in Finland) (Haagensen 2014: 73), and as in other Nordic countries, women make up the majority of the students. Hence, in 2013 women accounted for $64 \%$ of the graduates from universities in Iceland (Haagensen 2014: 74; see also figures from the Centre for Gender Equality Iceland, 2014). Despite women's overrepresentation among university students, women continue to hold a minority position in terms of university employees - representing only $25 \%$ of all professorships in 2010 (Heijstra et al. 2013).

It is furthermore important to note that, "many young men turn their back on university education, or even education in general" (Centre for Gender Equality Iceland 2012: 20) - possibly because Iceland does not provide financial support to students, but offers relatively large student loans (Haagensen 2014: 79). For the young men who can see opportunities for employment in the local labour market, it may appear more attractive to leave school early and obtain a relatively high income.

As described in Edvardsdóttir (2013), in Iceland, as well as in the other Nordic countries, education has been a political focus area as a way to create regional growth. Several studies indicate the possibility of using remote learning as a way to prevent depopulation in sparsely populated areas. Thus, most Icelandic universities today offer part of their studies as online long-distance courses. Although it was initially intended as an offer to students in peripheral areas, this form of study has provided new opportunities for all - both people who live in urban and peripheral areas; including being able to study without having to be in a classroom at certain times (Edvardsdóttir 2013: 74).

Edvardsdóttir (2013: 75) writes that in 2012 about 20,000 students in total were enrolled at Icelandic universities - of which about 3,000 were remote learners. Women make up about $60 \%$ of the total student body, and women's predominance is particularly high among remote learners (representing around 67\%). However, Edvardsdóttir argues that the increased access to remote learning is not necessarily a panacea that counters the uneven number of women and men who leave peripheral areas in the Nordic region. Thus, the results from her interview- 
based study among women in Westfjord who have followed a remote learning program, indicate that the kind of education that is available through this type of program tends to amplify existing gendersegregated educational choices and traditional views on gender roles in smaller communities. Thus, she highlights that among her informants there was a tendency to stick to family-based values and gendersegregated patterns, for instance in parenting practices - values which for a number of her informants had initially led them to choose to move to/remain in small communities. When these women then choose to pursue an education, they are partly limited by the choices they have (the type of courses that are offered), and also that they tend to choose areas of study which are dominated by women within education and care work - programs which are considered of "practical relevance" in their immediate environment. Edvardsdóttir writes:

Some of the women ended up learning what was not always their first choice, but rather something that can be studied through distance learning and was practical in the community they were living in (Edvardsdóttir 2013: 80).

In this way, the women's educational choices inadvertently reinforced a tendency in which women who do not accept traditional/conservative gender roles in the private and public sphere experience an enhanced urgency to move from small communities. Edvardsdóttir's informants argued that access to remote learning does not in itself discourage outmigration in small communities. Edvardsdóttir, therefore, concludes that small communities also need to retain and attract women who have more modern views on gender roles and women's employment opportunities in order to increase the diversity of perceoptions on women's and men's roles in society.

\subsection{Greenland}

Greenland is, according Haagensen (2014), the Nordic country that spends most public funds on education, and in 2005 Greenland's Landsting (Parliament) adopted a new education plan. Boolsen (2013) explains that the background for the plan was that the Government saw education as "necessary", and initial focus should be directed towards: 1) elementary school students with the goal of getting more young people to continue education after graduation (especially boys are "lagging behind"), and 2) unskilled workers under 50 years (mostly men) with the aim of providing them an education. In addition to increasing the 
proportion of the workforce with a formal education, the education plan also focused on a number of other aspects relevant to the focus of this report, including the inclusion and development of peripheral areas in the country, as well as upgrading the skills of the workforce to meet the changes in the global labour market (Boolsen 2010b: 3).

Overall, this report does not include a strong focus on elementary schools in the Nordic region. Nevertheless, in a Greenlandic context, the elementary school system is important, since common challenges in the primary schools influence the entire educational system in Greenland. This is partly due to the transitions between levels of education in the Greenlandic education system, which creates special challenges for young people, as geographic conditions often require that the young students move away from home to take a secondary education, let alone higher education. A report on Mobility in Greenland (Nordregio 2010) points out that a polarisation is taking place in Greenland. This is based on a survey which states that about half of the approximately 1,500 young respondents indicate that their highest level of education is primary school or not having completed primary school altogether. The report concludes that the polarisation manifests itself in a regional pattern, where the big cities attract young students, while a low level of education often characterizes the peripheral areas. It is clear that these communities represent a significant problem in a time when demand for education-related qualifications are increasing for instance in relation to new types of business activities in peripheral areas (Nordregio 2010: 141).

As for the younger children's schooling (up to 14 years) a report from SFI based on data from 2007 to 2008 points out that almost one third of parents do not believe that children learn in school, and about half of the parents in the survey are not entirely satisfied with the school system (Christensen et al. 2008). The same applies, incidentally, to some of the students themselves, hence, Budtz, Olesen and Pedersen argues in their study on Det gode børne-og ungdomsliv i Grønland (A good life for childrens and teens in Greenland) that some young people call for better quality in the education system, and express that they are worried about the consequences in terms of their future prospects as young individuals in Greenland (Budtz, Olesen and Pedersen 2011: 157). ${ }^{5}$

\footnotetext{
${ }^{5}$ In terms of the elementary schools, it is a problem to find qualified teachers in Greenland. This means that many in schools, teacher coverage (in terms of qualified teachers) is only 60-70\% (according to figures reported in Poppel 2010).
} 
A report from the National Institute of Public Health, Denmark (Pedersen and Bjerregaard 2011) on young people's well-being and upbringing in Greenland, points out that young people in their lasts years at primary school, "face many new choices, demands and opportunities which for a number of them means that they have to move far away from their safe environment with family and friends" (Pedersen and Bjerregaard 2011:3, own translation). Many young people find this decision difficult, and Pedersen and Bjerregaard refer to a previous study that found that young people between the ages 15-17 years have the lowest life quality among Greenlandic youth. They also report that, "young people who grew up in a small village often feel lonely in comparison with young people who grew up in a town" (Pedersen and Bjerregaard 2011: 8, own translation). The severity of the problem of having to move away from home at a very early age is underlined by the fact that public transport (in practice, often in the form of air travel) is very expensive, which often prevents frequent family visits (Poppel 2010: 56). This is the case both for young students who move to cities to enrol in an education or those who choose to leave their villages in search of work.

Precisely because a large number of young people do not finish an education after primary school, Greenland has politically focued on improving the transition from primary school to a further education program (Boolsen 2010c). The focus is particularly directed towards young people from small villages. They have so to speak, "grown up in the wrong place and gone to the "wrong" school in terms of being able to pursue their educational wishes" (Boolsen 2010c: 15, own translation).

From a gender perspective, it is particularly interesting regarding the transitions in the educational system in Greenland that Boolsen suggests that "girls and boys respond to setbacks (e.g. dropout) differently in the way that boys consider themselves and are considered by others as losers, while girls are described as victims" (Boolsen 2010c: 19). Regardless of gender, Boolsen finds that young Greenlanders tend to explain their education problems- and solutions at an individual level. She points out that this is problematic, since a number of the young people's issues are due to structural problems, which need collective solutions. A report on young people's well-being and upbringing in Greenland concludes - in line with Boolsen - that it is important for the young Greenlanders not be left alone with their everyday life problems - both inside and outside the educational system (Budtz, Olesen and Pedersen 2011: 153).

Another major challenge which characterises Greenland's educational system, concerns the imbalance between girls' and boys' educational level. Women in Greenland are generally better educated than men. This 
applies to secondary education, for example high-school, where $62.1 \%$ of the students who graduated in the period 1998/99-2002/03 were young women (Eistrup and Kahlig 2005: 210). In terms of higher education, Poppel (2010) argues that particularly women have made use of the new educational opportunities that arose in the wake of the Home Rule's effort to increase the level of education since the late 1980s (Poppel 2010: 49). In this connection, The University of Greenland was founded in 1987, and the proportion of women today exceeds men in all degree programs offered at the university (Poppel 2010: 53). According to figures from Haagensen (2014), in 2013 women accounted for $68.3 \%$ of all graduates from higher educations in Greenland.

In other words, educational choices in Greenland are highly segregated, in the sense that there is a, "general predominance of men in vocational training, and in turn, women dominate in both professional and higher educations" (Eistrup and Kahlig 2005: 210, own translation). Boolsen (2010b) also stresses that women in Greenland constitute "the educational elite", since women dominate the higher educations - this is despite the fact that there are more young men than women in the age group that would typically enrol in an educational program.

That women in numbers dominate the educational system should be seen in connection with other demographic factors. In Greenland, more boys than girls are born, but at the same time, infant mortality and suicide rates among boys are higher, and in the long term women in Greenland on average live over 5 years longer than men (Boolsen 2010b). It is also true that young women in Greenland typically begin their education later than men, and that the average age to initiate vocational training in 2002 was 25 years for women and 22 for men. This is most likely linked to the fact women in Greenland often have children at an early age (Boolsen 2010b; Eistrup and Kahling 2005: 211). A survey conducted by SFI from 2007-2008 also indicates that most women in Greenland are young, when they have their first child; thus, $30 \%$ of young women in the survey had their first child at the age of 18 or younger, and another $30 \%$ were 19-21 years old. 
When it comes to higher education, young people in Greenland are forced to think about moving. The vast majority of higher educations and technical training requires at least some schooling outside Greenland (often in Denmark) (Christensen 2013). Questions about education and mobility are also closely linked to demographic parameters - especially age and gender. Young people move most frequently, and for them the main factors are education, further training and upskilling (Nordregio 2010: 15). "Since the 1970s, and especially in the last decades, mobility has been very closely related to education" (Hansen et al. 2013: 203).

In addition to a significant correlation between gender and education in Greenland, a correlation between being born in a town versus in a small village is evident. Boolsen (2010b and 2013) emphasizes that it is especially young people from the cities who obtain an education, whereas young individuals from the villages are lagging behind. This factor is confirmed by Rasmussen et al. (2011), who also stress that the educational system in the small villages makes it difficult for young people to succeed in school and especially in a higher education. Their results also show that almost 50\% more women than men complete a primary school exam in the small villages (Rasmussen et al. 2011: 94). Thus, there is significant correlation between gender and place of residence and how well children in Greenland do in the school system - and as the report highlights, this provides "a good indicator of the problems children in the small villages are faced with, and it certainly affects the opportunities they may have to subsequently enrol in a higher education or career choices, which require further schooling" (Rasmussen et al. 2011: 99, own translation). If the educational level in Greenland is to be improved, Boolsen stresses that a number of practical parameters need to be considered. This includes: access to student housing, apprenticeships, the lack of qualified teachers, Internet access (at reasonable prices) and access to educational materials prepared in Greenlandic (Boolsen 2013: 74). There is, in addition, a need to focus on gendered factors, including the fact that primarily the boys are lagging behind. Furthermore, assessments of gendered patterns need to be co-considered in relation to differences in young people's educational opportunities, depending on whether they live in towns or small villages. 


\subsection{The Faroe Islands}

Primary schools in the Faroe Islands are geographically decentralized and administered by the municipalities. ${ }^{6}$ Gaini (2010) describes how the smallest schools often have only one teacher and fewer than ten pupils in total. He elaborates: "Faroese school policy has for decades been based on a principle which many today regard as outdated and inappropriate: the rule about not moving the children away from their home village before starting in seventh grade" (Gaini 2010:13). Gaini refers to a number of examples of this policy, which has led to extremely small schools - often implying great challenges concerning the quality of teaching. He writes further that among certain families there is a prevailing notion that elementary school:

... First of all (is) an institution which provides children with basic reading and writing skills, in addition to basic knowledge about culture, history and religion. Children must, according to many parents, learn the most essentials skills in school, and the rest is the responsibility of the family network. The children must not, in other words, "educate themselves away" from their family and community, and in the worst case scenario forget their Christian morality and identity (Gaini 2010: 16, own translation).

At the same time, Gaini states that in the Faroe Islands there is a group of boys who "grow up in a male dominated environment with strong antiintellectual values" (Gaini 2010: 17-18). Thus, Gaini finds that in certain families there is an absence of educational culture, and that particularly boys from these families are likely to stand back as losers in the future development (Gaini 2010: 25). Concerning the interplay between family and school, the Faroese Primary Education system states that parents "have the right to make decisions that concern the school's educational and cultural guidelines. Under special circumstances, parents can choose to home-school their children" (Gaini 2010: 13, own translation). If one compares this with Gaini's reference to boys who are affected by a male

\footnotetext{
${ }^{6}$ When discussing the Faroese educational system, it is difficult to ignore the question of language. According to Gaini (2011a), the Parliament's decision to elevate Faroese to be the teaching language in Faroese schools dates back to 1918. However, the teaching language today is still predominantly Danish; a contributing factor is that teaching material is often originally produced for teaching in Danish schools. And although Gaini argues that it is important for young people in the Faroe Islands to understand Danish if they want a pursue further education in Denmark, the input far from matches the reality of the children's everyday life. Gaini concludes that important questions about content and quality in the Faroese educational system is often overshadowed by the debate on teaching language.
} 
culture that is anti-intellectual, it could have consequences if especially these boys are taken out of public schools in order to take part in activities in the home. Thus, they might lose their abilities and desire to take a higher education. This in turn would reinforce the gender-segregated tendencies in the Faroe Islands.

The Faroese education system is of a high standard both in terms of primary and secondary education. Hence, the Faroe Islands have a number of upper secondary schools and vocational schools. There are three high schools in the Faroe Islands: one in Torshavn, one in Kambsdal (on Østerø) and on Suderø; and in Klaksvik and Torshavn there are vocational schools. ${ }^{7}$ There is a fishing school, a navigation school, as well as a school educating social and health care workers. In addition, the Faroese University was founded in 1965 and with its three faculties and about 100 employees and approximately 650 students (www.sefur.fo) is one of the smallest universities in the Western world.

Haagensen (2014) states 35\% of the population in the age range 15-74 years, has primary school as their highest level of education, $43 \%$ has secondary or vocational education, and only $22 \%$ hold a university degree. The numbers differs between the sexes. Currently, Faroese men are better educated than women, but this situation will change, since there are now more women than men enrolled in higher education in the Faroe Islands.

According Gaini (2005), in the 1960s the Faroese education system underwent large transformations, as many young people moved abroad - mainly to Denmark - to get an education. Where university educations previously were a privilege obtainable for a few young men from rich families, the situation is now such that many young people, especially women, leave the Faroe Islands to obtain an education. There are two mains reasons for this: many courses are not offered in the Faroe Islands and also young people in the Faroe Islands typically regard higher education as something one attends in Denmark. The female respondents in Knudsen's interview-based study highlight that in an effort to pursue further education, it is regarded a necessity to move from the Faroe Islands (Knudsen 2010: 34). A large proportion of young people who move to get an education will not return back home - this is especially the case for women.

\footnotetext{
${ }^{7}$ http://www.norden.org/da/norden-for-dig/uddannelse-i-norden/uddannelse-paa-faeroeerne/detfaeroeske-uddannelsessystem, date of access March 9th 2015.
} 
The changing conditions and opportunities for both men and women to take a higher education (for example in Denmark) are also important in regards to unskilled jobs. According to Gaini, these types of jobs are disappearing - even within the fishing industry - and based on a survey among children in their last years at primary school, he states that the vast majority of the 9th-10th grade students want to get an education (90\%) and about half plan to enrol in a educational program directly after finishing secondary education - and most with ambitions of achieving a mediumlength education (Gaini 2005: 16). Gaini also emphasizes that there are no geographical differences among the responses (questionnaires were distributed to all 9th-10th graders in 2003), but he does detect a small gender difference, as girls seem more determined to get an education. Regarding a question on young people's priorities in relation to choice of education and future job opportunities, it is interesting that only 5 out of 856 students in the survey stated that remaining in their local area was essential in terms of finding a future job (Gaini 2005: 16). The types of jobs they aim at were essentially the same in Torshavn as in the smaller villages. Many of the young people in the small villages considered it unlikely that they would return to their hometown after graduation. This was, in contrast, a more widespread perception among young people from Torshavn.

\subsection{The Åland Islands}

Åland has significantly fewer 15-39 year olds who are engaged in some form of education than in the other Nordic countries. Thus, only $24.9 \%$ of the total population of 15-39 year olds are recorded as being in the process of acquiring an education in the academic year 2013/2014 - this should be compared to an average of $36.9 \%$ in the other Nordic countries (except the Faroe Islands where there is no available figures) (Haagensen 2014: 72). It is important to note that these statistics not necessarily include students who are engaged in an education abroad. The observation of a relatively low educational level in Åland is, however, supported by Dahlström et al., "When it comes to education, the average educational level in Åland is still relatively low, and thus mis-matches remain in relation to demand in the labor market" (2006: 100). Unlike the other Nordic countries, the distribution of men and women who obtain a higher education in Åland is roughly equal: $52 \%$ men and $48 \%$ women among graduates in 2013 (Haagensen 2014: 74). Nonetheless, as the total number of people behind these figures is 71 , the number is so small that in practice it means that 37 men and 34 women obtained a university degree in 2013. 
Overall, there are slightly more women (25\%) than men $(22 \%)$ in the age group 15-74 years who have obtained a university degree. The level of education in Åland is generally increasing. By way of comparison: in the 1980s less than half of Åland's population had an education beyond primary school level. Today this number is significantly higher since 6 out of 10 have at least an upper secondary level education (ÅSUB 2013: 6). ${ }^{8}$

As seen in other Nordic contexts, a form of "stepping stone mobility" is found in Åland, in which young people gradually seek towards the major centres to obtain an education. "The same phenomenon (as in the Faroe Islands) occurs in Åland and its archipelago region, where most of the youngsters go to Mariehamn to get their upper secondary, and from there, leave for Sweden or Finland" (Hovgaard et al. 2004: 35). Despite the fact that Åland is formally an autonomous region in Finland, there are actually more students who go to Sweden than to Finland to study, because the main language on Åland is Swedish. According to Haagensen, there were 288 students from Åland in Finland in the academic year 2012/13 and 726 in Sweden (2014: 80). Dahlstrom et al. state that approximately $71 \%$ of all college students in 2004 chose to study in Sweden, and only 24\% chose Finland (Dahlström et al. 2006: 94). Åland has a polytechnic school where it is possible to receive vocational training, but the islands have no university - this educational structure might affect the gender division between women and men and their choice to leave the country to obtain an education:

\footnotetext{
Women represent the majority of Ålanders who study outside Åland. Conversely, the number of men who study in Åland is higher than the number of women. This applies to both those who are attending high school and in colleges and universities. Women choose to a higher degree than men to study outside Åland and this applies to most fields of study, although there are some exceptions mainly concerning health education. The explanation is most likely that the supply of training within in Åland is good and that it is women who also dominate this field in the labour market (ÅSUB 2014: 11, own translation).
}

Women in Åland have over the past decades achieved a higher level of education than men, and today it is only in the age group 65+ that men on average have a higher education than women (ÅSUB 2015: 9). There is a gap in the educational choices between young men and young women, which result in men still dominating the field of technology and science,

8 Ålands Statistik och Utredningsburå. 
while women to a large extent dominate in teaching and nursing. However, there are small signs of changes, "If you look at young people under 25 years today and their educational choices, there are small signs towards a more even distribution between men and women. This does not, however, apply to the areas of technology and science, where gender difference seems to be more or less locked" (ÅSUB 2015: 9). And in agriculture and forestry the gender patterns appear to be switching from a male dominated profession to being a female dominated profession (ÅSUB 2015: 9).

There is a clear segregation of the types of studies that young people choose as early as in high school. Young girls tend to choose language subjects and boys science and technology (ÅSUB 2015: 4). Moreover, the young people base their educational choices on the same gendered patterns as their parents' generation (ÅSUB 2015: 4). It is not possible to detect gendered patterns in the dropout rates among students in high school (ÅSUB 2015: 11). Overall, the situation is that "girls dominate in high-schools, social, health-oriented and humanistic education areas, while boys are heavily over-represented in technological education [...] The only clear trend is that girls and young women over time acquire a higher level of education" (ÅSUB 2013: 4).

Best Practice Case: West Nordic Master - Faroe Islands, Iceland, Norway and Greenland

The West Nordic Studies: Governance and Sustainable Management is a newly established multidisciplinary master's degree, which takes place in collaboration between the Faroe Islands, Iceland, Norway and Greenland. The program has its first intake of students in 2015 and offers courses from the autumn of 2015. The purpose of the program is to focus on the challenges for the coastal areas of Norway, Iceland, Greenland and the Faroe Islands (the Nora region), thus getting students to take an interest in the challenges and opportunities that the areas close to the North Pole face. The following universities offer this international and interdisciplinary joint master's program, which will promote exchanges between educational institutions:

Universities which offer The West Nordic Master:

- The University of the Faroe Islands.

- University of Greenland.

- University of Akureyri in Iceland. 
- University of Iceland.

- University of Nordland, coastal Norway.

The master's program focuses on issues in peripheral regions in relation to the subjects that students are presented with, but at the same time students will through exchanges obtain close first-hand knowledge about the areas they are studying, through visiting a number of these geographic locations. In addition to the various issues in peripheral regions that the education addresses, the master program also includes a gender perspective. This is expressed through teaching, where there will be a focus on incorporating gender perspectives in the literature used, through electives, as well as in the practical part of the training that is oriented towards businesses and public institutions. In addition, the climate and the environment are two major issues that the programme focuses on, which are also fields and professions in which a high proportion of women are employed. Indirectly, the program also has a gender dimension as the master's program should help to counteract the brain drain, in particular among young women, who more often than men move from these involved areas to get an education.

The program will take in its first students in the fall 2015, and has room for 25 students. The interim registration (to the Faroese University) currently (May 2015) runs at 10 in total, respectively 3 women and 7 men. Of these, two of the women come from the Faroe Islands and one from abroad. Among the men, three are local and four are foreign students.

Read more at:

- http://westnordicstudies.net/

Best Practice Case: The LOSA project - Norway

The LOSA project (Local Training in Corporation with the Labour Market) offers decentralized Internet-based education in rural communities where there is a low number of young people, and a small number of colleges (the alternative is often that young people must move away from home and into student accommodation or the like). Young people in rural areas can through LOSA take the first year of high school in their hometown via a digital learning platform, which includes education material (text, audio and video) related to the individual subjects/programs. Students receive Internet-based guidance from educa- 
tors, through MSN, Skype, email, video link and telephone. If young people make use of the offer in one of the small local colleges, the teaching in shared subjects takes place inside the classroom with the student's teacher. In municipalities without a secondary school, shared courses are offered through distance learning.

LOSA has been running for 10 years, and is offered to students in eight municipalities in Finnmark, and experience has shown that the program has led to more students having completed their schooling, compared to students who have had to move into student accommodation already as a 16-year-old. During the last 3 years, only 22 out of 200 students have not completed the program. The pass percentage of boys and girls attending the program is roughly equal. Of the 200 students who during the last 3 years completed the LOSA program from home, the number of boys is slightly higher than the number of girls. At the same time as the project is trying to address problems related to young people's educational opportunities, the project aims to build strong and productive relationships between school, municipality and businesses.

Hence, internships at local companies are a great part of the LOSA project. The number of students admitted in LOSA is adjusted in proportion to the number of available internship places in local businesses. The LOSA project is offered in relation to the traditional secondary education, as well as in relation to a number of educations within vocational training:

- Construction

- Design

- Electronics

- Healthcare

- Farming and Fishing

- Food and Restaurants

- Service and Transportation

- Technical and Industrial Production

Lessons learned from the program show that there continues to be a high degree of gender segregation in terms of courses elected respectively by girls and boys. This also means that slightly more girls than boys choose the general study preparation and then enrol in a university, whereas boys are more represented in vocational training.

Read more at: 
- http://www.nfk.no/artikkel.aspx?AId=12445\&MId1=1557

- http://www.masoy.kommune.no/losa-finnmark-et-av-norgesstoerste-skoleutviklingsprosjekt.273435-15290.html 


\section{Place Attachment, Everyday Life and Gender}

In this section, we will focus on different aspects of being young and living in one of the Nordic peripheral areas. The point of departure of this section is an understanding of the paramount significance of the actual places in which young people live in relation to their identity and subsequently also to their choices of education and career. The section is based on the idea that the identity question "who am I?" is closely linked to the questions "where am I?" and "where do I belong?", and that locations, or places, are vital in identity formation (Cuba and Hummon 1993; Howard 2000). The literature within the field suggests that to have a sense of belonging to a place and the people who live there are essential issues that young people must consider when they think about their future.

As stated previously, the young women of the Nordic peripheral areas seem to be more mobile and willing to move than the young men; this is partly due to the fact that the young women are more likely to aim for higher education. However, the research within the field also point to other aspects such as the existence of social and cultural institutions and the important part they play in young people's decision to stay in the peripheral areas or to move away.

\subsection{Sweden}

In a study on girls in Småland, Gunnarsson $(1993 ;$ 1994) concludes that many young women in the small villages experience that education, business and leisure activities mainly are aimed at young men, and that gender difference is created due to young women's limited opportunities for an active leisure life in the Swedish peripheral areas. She states:

In the context of hunting and forestry work, boys have the opportunity to participate in a community with males of different ages that the girls have no equivalent to [...] It is no wonder that so many girls choose to spend their leisure time at home, within the traditional female sphere, where they do not have to fight to assert themselves. Because they are not seen as much as boys in the public sphere, it is easy to believe that girls are idle. This is only half of 
the truth. Naturally, many girls would appreciate more collective activities, both outdoors and in indoor facilities, but let us not forget that they fill their leisure time with activities that are not as visible: talking on the phone; hanging out with family, their best friend and/or boyfriend; doing their homework; reading books; playing music; watching TV; cooking; train their dog, taking care of their horse; taking classes etc. (Gunnarsson 1993: 111; 117, own translation).

In line with Gunnarsson (cf. above), Waara (1996), who has studied young people in five municipalities near the Swedish-Finnish border, concludes that the surrounding nature and the possible leisure activities in the peripheral areas tend to create a gender gap. Thus, Waara, finds that young men in the peripheral areas often are more place-bound than young women, because the activities they are involved in (such as hunting, fishing and outdoor recreation) are more place-bound than those of young women. Similar conclusions can also be found in other publications on Swedish peripheral areas (cf. Stenbacka 2011; Werner and Annadotter 2013).

Svensson (2006) concludes (cf. above) that social background is a factor when considering why some young men have a particular sense of belonging to their local area. She finds that both the middle-class boys and girls who participated in her Söderhamn study share a clear belief of the necessity to move in order to get their preferred job or education. In contrast, young people from working-class homes generally prefer to stay in their local area; not necessarily because they believe that their area offers particularly bright prospects, but because they value closeness to family, friends and network and their particular lifestyle. Furthermore, they believe it is possible to find a way of supporting themselves even though they realise that their options are limited, Svensson concludes. However, while Trondmand (1995) concludes that factors such as weak labour market affiliation and low levels of education seem to be less important to working-class men in peripheral areas in comparison with the risk of losing their network and sense of security by moving, Svensson does not believe this to be an explanation for young men with working-class background being more place-bound than others. She concludes that the explanation is more likely that the young men consider their prospects to be brighter exactly by staying rather than moving - because they appreciate the culture and privileges they have as men in their local community and know that this would not be recognised outside the peripheral areas: 


\begin{abstract}
I believe that my study questions the assumption of the working-class youth's (mainly boys') "deep-rooted sense of home" emphasized in other similar studies. If anything, the working-class youth in my study do not feel at home/wanted anywhere other than in their family and circle of friends [...] The desire of the working-class youth to be able to stay in their native place may instead be seen foremost as an attempt is to keep "some" of the good life (Svensson 2006: 170; 169) (see also Waara 2003: 198, own translation).
\end{abstract}

According to Svensson, the desire of middle class girls to move is partly founded in their feeling of not fitting in in their local community. Their conclusion is that "they have everything to win by leaving Söderhamn. In larger cities, there are possibilities for development, more gender equality and better possibilities of becoming "somebody"'" (Svensson 2006: 168, own translation).

According to the two Swedish researchers Rauhut and Littke (2014), the so-called "macho culture" in the peripheral areas may be a factor which tends to have a more or less indirect impact on the young women's decision to move, because the young women find that the variation in their social and cultural options is insufficient, and because the space "offered" to them in the peripheral areas does not provide them with sufficiently positive value for them to remain there:

\begin{abstract}
The urban life-style with a better supply of female leisure activities, better paid jobs and better career possibilities in the modern and expanding sectors are definitely pull-factors; the traditional male leisure activities and "macho culture", unemployment, no social networks, differing preferences and ambitions beyond the traditional gender roles act as push-factors. So do factors as e.g. being bullied, assaulted and being sexually harassed during the primary and secondary school (Rauhut and Littke 2014: 16).
\end{abstract}

\title{
3.2 Norway
}

The fact that boys and girls may have different senses of belonging, and that their place-based integration and participation often vary, is also evident in studies from Norway, e.g. as concluded by Kaldal (2000) and Paulgaard (2002). In this regard, both state that something that causes many young women to move away from the peripheral areas at a relatively young age seems to be that the leisure activities and options available in these areas are mainly directed towards boys and young men.

Båtevik (2001) has conducted several studies on young people in the Norwegian peripheral areas. One of Båtevik's discoveries is that young people's sense of belonging in peripheral areas is dependent on gender, age, education plans and work plans (e.g. vocational training). Båtevik 
states that previous research in the field has suggested that young people in sparsely populated areas to a lesser extent than young people in more densely populated areas imagine a future in their local areas. The difference is particularly pronounced among the boys. In addition, Båtevik points out that the girls in sparsely populated areas differ less from the boys in more densely populated areas (Båtevik 2001).

Båtevik emphasizes that the boys in particular wish to settle in the local area in which they grew up, whereas the girls to a much larger extent wish to settle in the major cities. Båtevik points out that it is essential to study the young people's educational aspirations and priorities, when investigating who remains in the local areas and who moves away. Thus, the young people who have commenced or are contemplating commencing vocational training are much more likely to want to settle in their local area, whereas young people who wish to embark on further education will also wish to move away from their home municipality and settle in a major city. According to Båtevik, this trend has been verified by a number of studies on young people, education and gender. Although the boys are more likely than the girls to wish to remain in their home municipalities, the boys who wish to take a further education programme are also the boys who wish to move away. Thus, in addition to a general gender distribution (more boys than girls stay in the peripheral areas), there is also gendered correlation between the patterns of education and moving away (Båtevik 2001) (see also Orderud 2001 on belonging and sense of place).

Båtevik points out that it is not surprising that the young people who are positive towards their local areas are also more likely to imagine a future for themselves in their home municipality. According to Bătevik, it follows that the fact that girls seem less positively inclined than boys towards their home municipalities might indicate a connection between the options and activities offered for boys and girls by the municipalities, and suggest that some municipalities are more successful in offering a wide range of activities for the local youth. In his studies, Båtevik asked the young people in the peripheral areas what they find important that a local area has to offer:

It appears that a good social environment and good friends in the area where they live is important to most. Many also find it important that there is a basis for a job and a career. Many young people also find it important that there are good schools in the area. Even though many young people also want access to shops and a good selection of cafés, clubs etc., this is less often mentioned. There is a difference here between the responses from boys and girls. For instance, the girls are more concerned than the boys about access to good kindergartens. There is also some variation as to which qualities in the different 
peripheral municipalities the young people find to be most important. However, only minor differences exist (Båtevik 2001: 97, own translation).

Båtevik's research shows that a large part of the young people who have completed primary and lower secondary school have taken their first step towards moving away from their home municipalities by moving into student halls. However, Båtevik's research also points out that moving into student halls does not necessarily weaken the young people's attachment to their home municipalities. Båtevik introduces two different concepts, residential town oriented (hybelstadorientert) and hometown oriented (heimstadorientert) youth. Båtevik uses these two concepts to explain how some young adults in the peripheral areas relate and respond to their home municipalities. As demonstrated by a number of other studies, the boys were more hometown oriented than the girls.

Another Norwegian researcher who has studied belonging and gender is Rye (2006). Using a quantitative methodical approach, Rye has studied gender and life style patterns among young people in peripheral areas in Norway. His aim was to investigate the young people's reasons for moving away from the peripheral areas or for remaining - supplementing with quantitative data in a field which, according to Rye, has mainly been founded on qualitative data. Rye points out that certain differences may be spotted between the moving patterns of boys and girls and their preferences for living in urban or more peripheral areas. According to his studies, says Rye, there is an 11 per cent difference; he also finds and that the larger number of girls preferring the urban areas, compared to the boys, is only detectable while they are in their 20s; after this time, the difference is reduced considerably. (See also Pedersen 2013 for further quantitative research on moving). Thus, Rye points out that:

The attraction of the cities seems to fade in later stages of life, regardless of gender. Only small minorities of the rural young preferred urban settings for life when raising children (12\%) or when retired (9\%). Here there are no statistically significant differences between genders at all. Thus, the claim that rural girls are more urban in their residential preferences than rural boys finds only qualified confirmation in the quantitative material employed in this article. Insofar as there are gender differences, these apply to the young adult period of life only. Neither boys nor girls seem to have lifelong preferences for urban life (Rye 2006: 204).

Rye (2006) points out that in the light of his study, it can be concluded that boys and girls have largely the same desire to return to their local areas after completing their training or studies. Rye emphasizes that the problem of young adults migrating to urban areas should not be seen as an 
indication that the young adults do not wish to settle in peripheral areas; this applies to both boys and girls. However, educational preferences cause young adults to move away from the peripheral areas, and once they have become established in the cities with an education, jobs, family etc., it is very difficult to break up and return to their home municipalities.

Paulgaard $(2002 ; 2015)$ has studies gender, youth and identity particularly in peripheral areas in Norway and has focused on, among other issues, local, regional and national identities. Paulgaard argues that narratives on local identities in the peripheral areas of northern Norway may have a limiting effect and may create the myth of a certain type of person who is very old-fashioned. This may make it difficult for young adults in these peripheral areas to identify with their own local areas. According to Paulgaard, this may cause young adults to make a distinction between being modern and living in northern Norway.

Paulgaard also points out that it may be difficult for young adults in the peripheral areas to leave their safe and well-known local area with close family relations and a social safety net, and that this may cause some young adults to remain in their local areas. However, Paulgaard points out that unfulfilled expectations as regards job opportunities in the peripheral areas may create a conflict between the young adults and their families:

In the peripheral areas of the High North, as well as in other places, home may represent safety, familiarity and often both social support and other types of support from the family. The conflict between the experience of familiarity and safety at home and lack of opportunities for work create tension and discomfort. The young men may see and desire the opportunities that might be available to them, but for most, such opportunities turn out to be illusory and beyond reach. Unemployment implies that the anchor for traditional masculine identity might be challenged, as well as the potential to provide for oneself (Paulgaard 2015: 214).

In line with others (Paulgaard, Rye and Båtevik) Bæck (2004) points out that girls in the peripheral areas are more likely than boys to move away from their native place. In her studies based on students aged 15 to 19 from 19 schools in 11 municipalities in Troms (the second most northern region in Norway), Bæck shows that the girls are more likely to have a close social relation to their local areas; however, at the same time, the girls are more likely than the boys to move away from their native region. Bæck also points out that among the girls a difference can be traced as to who leave the region. To a large extent, this depends on social belonging or a wish to engage in different leisure activities offered, for instance, by a major city. According to Bæck, this difference is not seen 
among the boys in the peripheral areas (Bæck 2004). Bæck points out, that for both genders the main reason for moving away from one's local area is the availability of job opportunities. This causes many young adults to ignore their sense of belonging in favour of job opportunities in other areas - often in major cities (Bæck 2004).

Sørlie et al. (2012) have prepared the report Why move? Why stay? based on the key findings from a study of "Bo og flyttemotivundersøkelsen 2008" (Residence and mobility motive study 2008), which was completed by Statistics Norway (SBB) and the Norwegian Institute for Urban and Regional Research. The report is the first national study in Norway since 1972 attempting to identify motives for migration. In regard to a gender perspective, studies by Sørlie et al. suggest some of the same conclusions as pointed out by Paulgaard and Bæck et al., namely that:

While men state their reasons to both moving and staying with work, the women's arguments are more related to family. This is the greatest difference between the sexes. The difference is largest for those who return to their origin municipalities in the periphery regions. Work motive is prominent in the younger life phase. Much of this is migration in centralized direction (Sørlie et al. 2012: 19-20).

\subsection{Denmark}

Belonging is essential for the peripheral regions as this is the main reason for some young adults to choose to remain in their local areas, and for some to return to the local areas of their childhood. At the same time, this is also a factor for the individuals who choose to leave their native region for good. The literature in the field points towards a trend that boys in Danish peripheral areas have a greater sense of belonging than do girls. There are several reasons for this; one is that boys are often closer attached to the living patterns represented by their father's. For this reason, they often find it natural to take up the same occupation as their father's; occupations which are deeply rooted (historically and geographically) in the local communities (Ebbensgaard and Beck 2009; Yndigegn 2003a and 2003b; Hansen 2011 and 2014; Bloksgaard, Hansen and Faber 2014).

In Yndigegn's study of young adults in the Danish-German border region, he concludes that belonging is of great importance to young adult's dreams concerning education and future work life (Yndigegn 2003a and $2003 \mathrm{~b})$. He concludes that young adults living in peripheral areas often either plan their lives on the basis of the opportunities offered in their 
local area or outside of their local area. An overall conclusion by Yndigegn is that among the young adults in the peripheral areas, there is a general willingness to move, at least temporarily, for educational reasons. However, gender differences exist, concludes Yndigegn. To some of the young adults attending vocational training (more often boys than girls) it applies that "they are planning their lives in a context which enables them to be trained and to expect to find a basis for a livelihood in the local context" (Yndigegn 2003b: 402, own translation).

Svendsen (2007) concludes that, in particular, occupational communities relating to local work traditions are identified as motivation when young men choose to remain in the peripheral areas. To capture the importance of such local (masculine) networks, Svendsen uses the concept of place-bound resources. Place-bound resources cover for instance local networks; these might be seen as a type of social capital which constitutes a social resource for the individual, in line with other types of capital.

Beck and Ebbensgaard (2009) have conducted a study on young adults in a peripheral area in Denmark. Their overall conclusion is that there is "a collision between, on the one hand, the values and attitudes (the young adults) have acquired as regards a good life in a peripheral area and, on the other hand, the career oriented identity markers of society stating that you are only somebody if you can make a career for yourself" (Beck and Ebbensgaard 2009: 275). In their study, Beck and Ebbensgaard also find that among the boys, there is a larger number with a local orientation. In their opinion, this must be seen in the context that the boys make a more gender traditional choice as regards education and occupation, and that they are more likely to reflect the occupational choice of their father's than are the girls. They conclude that there are more boys in the peripheral areas who have a stay-at-home identity in contrast to the girls in the peripheral areas who are more likely to have a nomad identity, because they are more mobile and extrovert. Beck and Ebbensgaards write:

For the boys, the choice of secondary education is often gender specific and dependent on local traditions or the family-related cultural environment. [...] Boys in the peripheral areas are often attached to living patterns represented by their father's, and they find it natural to adopt the occupational patterns which are prevalent in the area in question and perhaps to remain in the place where they always lived. In other words, more stay-at-homes are found among boys than among girls [...] The girls seem to be more mobility and modernity oriented than the boys. [...] The girls are nomads and globally oriented and do not wish to adopt the traditional female role (Beck and Ebbensgaard 2009: 104, own translation). 
Hansen $(2011 ; 2014)$ has conducted fieldwork among young adults in Hirtshals in the northern part of Denmark. Hansen concludes that in this area, young adults experience a conflict between their sense of local belonging and, for some of them, the wish to have an education, which, in many cases, would require them to leave the area. In both cases, the local occupational traditions are essential for the young adults' choice of occupation and education. Hirtshals has a long and flourishing fishing tradition, which is also the framework of a special sense of community developed through generations. According to Hansen, this community and the relationship to the local work traditions has a clear gender dimension. Hansen writes:

The fact that fewer young men than women in the local community acquire secondary education reflects in many ways that boys to a smaller or lesser degree relate to the sea and the harbour (to the occupational traditions of the local area, ed.); in other words, to the logic and practicality which are historically rooted in fishing. Girls are not expected, in any way, to be inclined towards the harbour. This would be a last resort and generate a different and much more pronounced social condemnation than among the boys, who should not be afraid of getting mucky trousers (Hansen 2011: 82, own translation).

Hermansen (2011) has written about the issues in a peripheral area in the southern part of Denmark, i.e. Horne Land in the island of Funen. On the basis of his study of young men and their attachment to their local area, Hermansen concludes that the sense of community in the peripheral areas is very attractive to young men here - and more so than it is to the young women. He continues to conclude that the constructions of masculinity of young Danish peripheral men often include the idea that "rural life is more fitting for men" - and simultaneously that this renders the young women's choice of moving away from the peripheral areas understandable (due to lack of opportunities for young women). Hermansen further concludes that the leisure activities in the peripheral areas create a gender gap, because these activities are largely characterized by traditional male interests.

Faber et al. (2014) conclude in their study of young people in Northern Denmark that boys in the peripheral areas have a slightly greater sense of belonging to the place they live. All things considered, this means that there is more at stake for them if they move away from their home, for example in order to pursue further training elsewhere. The boys in the survey have a greater sense of belonging than do the girls, both to their local community, town/local area as well as to their region. The boys are also slightly more positive about the social relationships in 
their local community than is the case among the girls; likewise, they are more positive about the possibilities for leisure activities, education and job opportunities as well as for finding a partner in their local community. Thus, a large proportion of the young men in the study considered it as something positive to stay in their local community - they do not see themselves as "losers" who have been left behind; on the contrary, they attach positive value to staying.

\subsection{Finland}

Family relationships and belonging to local areas have great significance in the peripheral regions in Finland; according to Peltomaa (2013), this is particularly evident in the northern regions of Finland such as Lapland:

In the north, the first thing people want to know is "whose daughter/son are you?" and not "what do you do for a living?", which is the more common question in the south [...] Membership in a known family makes you belong as "one of us" (Peltomaa 2013: 161).

Westman (2005) points out that Lapland is a place of great contrasts, both geographically and culturally. Lapland is geographically located in a border region and shares borders with not only other Nordic countries, but also Russia where the living conditions and culture in many ways differ from those of the Nordic countries. The close geographical proximity to Russia, Sweden and Norway in many ways means that Lapland as a region is a multicultural place where many peoples coexist. At the same time Westman (2005) emphasizes that Laplanders is a population group who refer to themselves as the only native population of Northern Europe with old traditions and customs that continue to be upheld. Because of its location, Lapland is part of the Nordic Periphery (sparsely populated) and located far from the urban life in the capital of Helsinki. At the same time, Lapland is closely linked to the surrounding world and the global economy, mainly because of ski tourism (Westman 2005). Although the region according to Westman largely meets the conditions necessary to be labelled a multicultural society, Peltomann (2013) emphasizes that the population of Lapland in many ways represent a closed community where belonging is an important factor in order to become accepted in local communities:

In Lapland, there is even a specific term for those who were not born here: junantuoma, literally "those brought by train". It is very hard to get rid of this label and to become accepted as one of the villagers, as one of us. Women es- 
pecially feel that if you are from somewhere else it is even more difficult to be seen and heard as a local. In Lapland, divisions are traditionally made by gender, ethnicity, and political or religious views, but it also seems that having moved to a village from somewhere else is nowadays another way of being an outsider (Peltomaa 2013: 159-161).

Peltomaa's study emphasizes gender as a specific factor in relation to belonging; this is part of a discussion about gender and geographical belonging which is also evident in the other Nordic countries.

Lundholm et al. (2004), who have examined the reasons for moving in the Nordic countries, point out that for Finland, in particular, lack of employment is the most significant reason for why people move away from the peripheral areas, and that this constitutes a more important factor than lack of educational opportunities.

Westman (2005) has also studied men and women's sense of belonging to peripheral areas of Finland with a focus on gender, the welfare state and the labour market in the peripheral areas. According to Westman and a number of other researchers in the field (see e.g. Paakkunainen, Peltomaa and Keskitalo-Foley Seija), it is mainly women who leave the peripheral areas of Finland. This image is very much in line with the research within the field of the other Nordic countries. According to Westman (2005), one of the main reasons for women to leave the Finnish peripheral areas is limited job and educational opportunities (Westman 2005). Westman argues that the peripheral areas gradually lose women to the cities due to globalization and changes in the local, national and international labour market. This causes problems for those men who choose to remain in the peripheral areas in relation to finding a partner and starting a family and, at the same time, it has consequences for gender relations (the local gender contract). According to Westman, the young women are more mobile and willing to move than the young men:

\footnotetext{
All in all, women often leave Lapland on the mental and bodily level. Men remain. For example, if a woman is a mother, she will stay with her family, but she wants to push her children out of the North, especially from small villages, towards education and therefore better opportunities in life. Young women are more eager to leave than young men (Westman 2005: 136).
}

Another area in Finland where belonging is of great importance for the population flows is Kainuu, which is located in the middle of Finland and borders up to Russia. Kainuu is suffering the most drastic decrease in population numbers of all regions in Finland. The local population in Kainuu is spread over a large area, and it is one of the places with the 
lowest population density in Europe. Infrastructure and road conditions make commuting for work very difficult, and the region has an increasingly ageing population (Dahlström et al. 2006). Dahlström et al. (2006) emphasize that sense of belonging is essential to whether young people will remain in the cities or return after graduation. It is often social networks and relationships that make it possible to find employment in the region, particularly the first job, and thus belonging becomes a significant factor in regard to the possibilities of settling and finding employment in the peripheral areas of Finland. This is illustrated in the studies of those who leave and those who move to Kainuu conducted by Dahlström et al.

\footnotetext{
Most of the interviewees had experienced migration in one way or another. There are locals born in Kainuu that were returning from studies or coming back because of job opportunities associated with family ties, and there are also newcomers attracted by job opportunities or family relationships. It seems then that the appreciation of personal roots and a feeling of belonging through their family and/ or professional ties play a special role for the interviewees. The family social networks function as a "pull" factor for the region, their capitalisation being more likely when there are also well functioning professional networks. It can be argued that a positive attitude together with access to local family and professional networks are crucial for entry into the regional labour market (Dalström et al. 2006: 70).
}

\subsection{Iceland}

Bjarnason and Thorlindsson (2006) find that the small communities in the peripheral areas of Iceland tend to lose the young population - women in particular - and as a result old men are often over-represented in these areas. They conclude that girls in the peripheral areas are 1.6 times more likely than boys to respond that they plan to leave their local area in the future. According to Bjarnason and Thorlindsson, this trend has been amplified over the past decades; while $60 \%$ of young people in the countryside in 1992 expected to leave their local communities in the future, in 2003 this figure had increased to $69 \%$. They also found that boys were less likely to plan to move with a factor of 0.68 in 1992 and 0.64 in 2003 in other words, it seems that the gender gap is widening over the years, albeit the expectation of moving might not match the actual moving patterns. Furthermore, the desire to move increases in correlation with the parents' educational level - the higher the educational level of parents, the more likely it is that their children are planning to move away from the area. And the better the children do in school, both academically and so- 
cially, the more likely it is that they want to move away from the area. At the same time, research shows a difference between the small communities that are primarily dependent on fishing and those primarily based on agriculture - there are more young people who plan to move away from the small agricultural communities than from the small towns that are dependent on the fishing industry.

Bjarnason and Thorlindsson's overall conclusion is that the more the young people identify themselves with their local area, the less likely it is that they want to move away from the area. They are more hesitant when it comes to explaining why the girls are more likely than the boys to want to move away, and they give two explanations: a strong and a moderate interpretation of the data they have collected among the young Icelanders:

A strong interpretation of these results would be that job opportunities are the only relevant factor in gendered migration, and that issues such as e.g. limited recreational opportunities or strict expectations of normative conformity do not matter in this context. A more moderate interpretation would be that the job opportunity structure serves as a proxy for various other factors, and that not wanting to work in the primary industries may in itself be the result of a whole host of gendered considerations (Bjarnason and Thorlindsson 2006: 298).

Bjarnason and Thorlindssons thus suggest that the girls find that the leisure activities in the peripheral areas tend to be oriented towards the boys/young men, however, they also point to the fact that ideas and expectations about gender in small communities also play a role:

Many young women find the closeness of small communities to be claustrophobic [...] The informal networks of social support that characterize many rural communities involves substantial unpaid work that women are expected to perform. These factors could be expected to predict a greater migration propensity among females, independent of educational and occupational opportunities (Bjarnason and Thorlindsson 2006: 298).

Karlsdóttir and Ingólfsdóttir (2011) have also conducted an interview study among women outside the metropolitan area - more specifically, in the small town of Húsavík on the north coast of Iceland with approximately 2300 inhabitants. The focus of their study is women who - in spite of the general trends - have chosen to remain in the small community, and the reasons they indicate as explanation. They conclude that "(f)amily and other social relations clearly emerged as one of the most important factors influencing the women's decision to stay in the village in spite of economic decline" (Karlsdóttir and Ingólfsdóttir 2011: 171). Social ties were most 
often indicated as a reason to stay, whereas the lack of jobs pulled in the direction of a desire to move away. However, according to Karlsdóttir and Ingólfsdóttir, the local men and women apparently had different strategies with regards to responding to changes in the local labour market:

While men who lost their jobs in the village sought similar jobs, either locally or further away, women have been more likely to change sectors. For example, according to the women interviewed, many women who previously worked in fish processing factories had begun working for the public sector, either at the hospital, in welfare services, at the kindergarten, or in the schools (Karlsdóttir and Ingólfsdóttir 2011: 171).

According to their informants, several women from the local community had also chosen to take a new education or a continuing education programme in response to changes in the labour market. Similar trends are evident in other studies; Rafnsdóttir and Ómarsdóttir summarize their findings as follows:

The women (in Iceland) are moving away from the so-called peripheral areas to a greater extent than men, and they are less likely to return; among other reasons, this is due to the monotonous occupation opportunities, few education opportunities and conservative attitudes to gender roles. This development has resulted in a skewed age balance and gender balance in the population (Rafnsdóttir and Ómarsdóttir in Rafnsdóttir 2010: 92, own translation).

The men in the peripheral areas, on the other hand, often end up commuting or as long-distance workers rather than retraining for other sectors, Karlsdóttir and Ingólfsdóttir (2011) conclude. Karlsdóttir and Ingólfsdóttir point to this pattern being in line with findings in previous research which show that "the perception of activities related to renewable resource exploitation, customarily performed by men, seems to be "sticky", in the sense that the prevailing discourse with respect to men's employment has difficulty in moving on from what were once key economic activities" (Karlsdóttir and Ingólfsdóttir 2011: 169); whereas women seem to be more adaptable in their search for work. They therefore conclude that while the men's strategy seems to be mobility, women's strategy is often flexibility - with the side effect that the unequal distribution of the responsibilities in the home will be even more skewed, since the men in practice are absent more of the time.

In the article Place and Space for Women in a Rural Area in Iceland, Edvardsdóttir (2013) examines what a number of Icelandic women from the Westfjords of Iceland have gained from following a distance learning programme at one of the Icelandic universities. She concludes that women have used their training to enhance their status and position in the rural 
communities, but also that their education has not contributed significantly to change their "action space" in the local community, nor has it changed the basic economic power structures prevailing in small communities. Her study thus points to that the Icelandic women "all felt their communities to be male dominated, with male values and beliefs; the smaller the community, the more male dominated it was" (Edvardsdóttir 2013: 81). This male supremacy in the small communities is primarily attributed to men's dominant role within the fishing industry, which is often the sustaining profession in many small communities. As described by Edvardsdóttir:

The women identify the fishing industry as the largest industry and at the same time the most male dominated industry in the area, where money and power go hand in hand. They are excluded from that industry and therefore feel their community to be male dominated. That factor is one of the reasons women move to more urbanized areas [...] The women also feel that political activities are male dominated and don't want to go into that field of sphere (Edvardsdóttir 2013: 82).

A number of studies (cf. previous sections) suggest that a possible decision to move away from one's local area is closely linked to family circumstances. Edvardsdóttir writes:

Families stay in rural areas as long as the male partner has a job, even if the economy in rural areas is monotonous. When one loses one's job, it might be difficult to get another one but it is more important that men have jobs. Research has shown that the lack of diversity in the economic sector plays a big role in out-migration from rural areas (Edvardsdóttir 2013: 83-84).

However, families in Iceland are changing, and this has a major effect on the development of the small communities. In an article by Wildy et al. on the conditions of the small schools in Iceland, the following description of the current development trends appears:

Our community is changing. In the past, families had four or five children. The youngest children of these older families are now graduating from the school. As they move away for further education, they don't return because there are few work opportunities. Now younger couples have fewer children and they, too, are finding work elsewhere (Wildy et al. 2014: 111).

The debate about the difference between rural and urban areas and the opportunities women experience that they have in various contexts is put into perspective by Edvardsdóttir's interview-based study; it truly underlines the fact that centre and periphery can be relative concepts (for a discussion of this, see also Faber and Pristed Nielsen 2015): 
In the women's mind, the villages around Iceland are urban areas and the countryside is rural. To be able to call a community rural or urban depends on the status of the service that can be found there. If there are all the necessary service institutions, e.g. a pre-school, a compulsory school, a health clinic with a doctor and/or a nurse, various shops that sell food and/or clothes, a town hall and a bank, the women felt they live in an urban community (Edvardsdóttir 2013: 82).

\subsection{Greenland}

According to research, local network is of paramount importance to young people's decision to stay or move away (cf. above). This is true of all the Nordic peripheral areas, in particular of Greenland, and this is also emphasized in the report Mobilitet i Grønland (Mobility in Greenland) prepared by Nordregio (2010). The report states that family network may compensate for poor living conditions and encourage young people to stay even though they might have greater opportunities if they moved (Nordregio 2010: 15). In contrast to most of the research within the other Nordic peripheral areas, research does not find the young men in Greenland to be immobile. On the contrary, research shows that the village population in Greenland, in particular the young men, often have a mobility pattern that might be characterized as a "bungee jumping" (Pristed Nielsen 2015). In other words, the young men - even though they are closely attached to their communities and maintain a strong sense of belonging - are mobile at the same time, because they often move temporarily in successive time periods. Due to this type of moving pattern, the young men are able to maintain their sense of belonging and network in their home village, while at the same time taking advantage of some of the new income opportunities in Greenland; these include work in the extractive industries or other types of jobs with a rota system (working for a specific time period away from home followed by a leave period) or short-term project employment. This pattern of shortterm, temporary employment is particular evident with regards to men within the low-income bracket in Greenland: "More men than women move short distances, typically within regional labour markets, to obtain shortterm or long-term employment" (Nordregio 2010: 65, own translation).

In contrast to this type of belonging, people born either outside Greenland (for example in Denmark) or in the towns, are more likely to move permanently, rather than move back and forth in the aforementioned "bungee jumping" patterns. Thus, the differences in the type and degree of mobility are not only connected to place of origin, but also to gender; more detailed responses from the questionnaire survey in Nor- 
dregio's report illustrate that the gender differences can be related to various notions of social norms and obligations for women and men respectively. The report concludes as follows:

\footnotetext{
While the men - in particular the older men - state temporary jobs as an opportunity to supplement income from for example whaling and fishing and thereby maintain their traditional employment, the women to a greater extent see part-time jobs as their opportunity to supplement the family income and thereby maintain social relationships in their community. The Greenlandic men thus view their home as a base they can leave for a shorter or longer period of time, while women feel more obligated to maintain this base function (both at the individual and social level). When Greenlandic women want to leave for work reasons, they will usually move permanently in order to "establish a new base at their chosen place" (Nordregio 2010: 17, own translation).
}

There is much literature on young people in Greenland including research on: youth life in a global age in particular focussing on media impact on young adults in Greenland (Rygaard and Pedersen 2003); living conditions, welfare and health of young adults in Greenland (Curtis et. al 2004); urban youth life in Greenland (Medonos 2010); and social problems of young adults in Greenland (Christensen 2013). However, the research within the field virtually has no emphasis on the importance of gender.

Similar to other Nordic countries, research shows that young people's sense of belonging in Greenland also is linked to their leisure life. The Nordregio report shows that while sports facilities are important to the urban population, outdoor activities in nature and arts and crafts are more often mentioned as leisure activities in rural communities (Nordregio 2010: 16). In an analysis of young people's everyday life in Greenland Rygaard (2003) states:

Many of the cherished joys of youth life such as cinemas, clubs and discotheques are not available in most communities. In many places there is not even a grill bar, a clothing store or a place to rent videos. At best young people can meet at a sports hall. On the other hand, one certainly is able to find other interesting leisure occupations such as sailing, fishing, kayaking, skiing, dog sledding, camping and hiking, which adventure-seeking youngsters in the metropolitan areas may look upon with envy (Ryggard 2003: 294).

Rygaard distinguishes between two different types of Greenlandic youth life: global style youth life (characterized by activities such as clubbing, shopping and dining out) and local style youth life (characterized by activities such as Greenlandic polka dancing, hunting, fishing, riding snowmobile, skiing, kayaking or leatherworking). Rygaard's analysis is based on quantitative data, but does not consider any gender differences. Thus, it 
remains unexplored whether it is also true for Greenland that the leisure life and the possible activities contribute to the young men being closer connected to their local community than the young women (cf. above).

In addition to the themes that cut across the various national contexts in this review, there are also specific themes in the literature important to specific national contexts. This applies to Greenland in particular with regards to high suicide rates, unwanted sexual experiences and addiction problems, which obviously affect the everyday life of Greenland's children and youth and thus their education. Both Poppel (2010) and Eistrup and Kahlig (2005) emphasize that Greenland has particularly high suicide rates, especially among young men. In their study of lower-secondary school leavers, Pedersen and Bjerregaard (2011), on the other hand, find that it is primarily among the girls that a pattern of suicidal behaviour and mental health problems is exhibited. And although a significant decline in suicide attempts among girls since 2004 can be detected, they argue that "the occurrence rate among both boys and girls is still very high - nearly one in ten boys and every fourth girl have attempted to commit suicide" (Pedersen and Bjerregaard 2011: 9, own translation). Furthermore, Pedersen and Bjerregaard argue that rather many of the young people in their survey, which comprised nearly half of all pupils in 9th and 10th grade in Greenland in 2011, had had unwanted sexual experiences with a peer or had been sexually abused by adults. In addition, half of the 15-17year-olds reported addiction problems in their families and "one in three of the young adults has experienced alcohol addiction in their immediate family, and one in five had a parent with an alcohol problem" (Pedersen and Bjerregaard 2011: 12, own translation). According to the study, other forms of addictions were also widespread, and it is concluded that many young people in Greenland experience family members' addiction problems in their childhood. By contrast, the Greenlandic youth drink less alcohol and are less often drunk as compared to the Danish youth (Pedersen and Bjerregaard 2011: 14)(see also Christensen 2013).

\subsection{The Faroe Islands}

According to Gaini (2011a), people on the Faroe Islands have a strong sense of belonging. The local identity is very strong, and sense of belonging constitutes "a major pillar in the subjective self-perception and cultural identity of any Faroese person. Land is, in short, identity's nexus, even though this is rarely apprehended as a qualitative cultural property giving "meaning" to the Faroese who live in the beginning of the 21st century" 
(2011a: 140). Gaini further writes that even if the Faroese sense of belonging has changed since the end of the 20th century in a way that challenges the notion of the place as a single geographical entity, the idea of the Faroe Islands as a place remains to be a strong mental construction with cultural significance for most Faroese. However, many Faroese leave the islands without considering to return, because they believe the small communities on the islands "to limit their personal visions for a (late) modern lifestyle" (Gaini 2011a: 158) (see also Gaini 2003).

Gaini further concludes that most portrayals of the Faroe Islands and its communities can be considered exotic narratives; he emphasizes that the islands have never been as isolated as they traditionally have been represented in literature, and that the islands have always been influenced by their Northern European neighbours and have been a part of international networks. However, global trends in recent years have contributed to the speed and regulation of the ongoing processes in the Faroe Islands, and the social infrastructure has changed dramatically during the 20th century, in particular as a result of the emergence of the modern centralised "mass society" based on new national, cultural and political institutions. According to Gaini, a revival of interest in the traditions and cultural activities in the Faroe Islands has been evident in recent years, and this has led to a strong cultural life rooted in the local community (Gaini 2011a). Nevertheless, the Faroese youth follow the global trends regar7ding education and career choices (Gaini 2005: 13). Gaini emphasizes that the lack of organised leisure activities (except church attendance) is a problem for young people in the smaller villages and states that this lack of activities may be an important factor in young people's decision to move to Tórshavn or, alternatively, completely to leave the country (Gaini 2011a: 180). Gaini compares the situation of the Faroese youth to that of the youth in Northern Norway. He argues:

Both regions have today youth populations with approximately the same interests and values and "central" urban youth, even if they keep the strong connections to nature, family and local society [...] They want to make a future in their home region, but they also want the "fancy" high status jobs normally associated with large Western cities (Gaini 2005: 23).

However, Gaini draws no conclusions on gender in this text. With regards to the values inherent to the choice of staying in the small communities, Weyhe (2011), on the other hand, argues that "the findings in the Faroe Islands show that it is mainly men who choose to stay in the villages, partly because men are more concerned with upholding the 
traditions and values of traditional village life" (Weyhe in Baagøe Nielsen 2011: 252, own translation).

In addition, Knudsen (2010) suggests that the lack of a well-developed social and technological infrastructure is also part of the explanation of why Faroese women are moving away from the villages:

The social and technological infrastructure in place in most cities and urban areas makes it easier for women to combine career and family life [...] The better these opportunities are, the more attractive the area is for women to live in (Knudsen 2010: 19, own translation).

Hovgaard and Kristiansen (2008) also argue that sense of belonging in the Faroe Islands is changing as the new technological developments make it possible to move from one place to another - whether physically or virtually. Therefore, this development enables new lifestyles, where the Faroese men in particular - can choose to live in one place and work in another, they argue. In a later article, Hovgaard (2015) describes how new developments in communications technology have changed the everyday life especially for Faroese long-distance workers, typically men who are employed in the merchant fleet or offshore industry, who now have the opportunity to retain a sense of belonging to their community due to the technological development. Hovgaard writes:

\footnotetext{
Because family decisions, family planning and family obligations can be managed by cell phone, mail, Skype and Facebook, you may be part of your home even while away. The mariners are talking with their family nearly on a daily basis, and the time they have for Internet surfing means that they claim to be more up-to-date on local issues than family and friends permanently there (Hovgaard 2015: 182).
}

With regards to explaining why Faroese women are moving away, not just from the villages but from the country entirely (often to Denmark), Knudsen argues that their primary concerns are economy considerations and access to education. However, an entirely different issue also seems to be at stake. Her study shows that women "in many cases have greater confidence in the authorities and public administration in Denmark and seem to be reasonably aware of what rights [...] they have in Denmark" (Knudsen 2010: 25, own translation). Based on several interview examples, Knudsen states that collecting social benefits for single mother's often become stigmatising for this group of women, for example. She also points out that some of the Faroese women find that they are at a disadvantage in the Faroese society because they are women; and that the Faroese society does not safeguard their interests. Knudsen 
thus argues that some of the formal structures in the Faroe Islands are weak and that they are not "able to meet the demands and desires of the citizens, women in particular" (Knudsen 2010: 26, own translation).

In a discussion of the Faroe Islands as a society without major social divides, ${ }^{9}$ Jacobsen and Jákupsstova (2005) emphasizes the fact that the Faroese welfare model can be characterized as family based, and that the development of public welfare institutions such as kindergartens etc. have contributed to the marginalization of women's interests in the Faroese society. Examples include that the right to maternity leave with pay was only introduced in 2001, and that in several municipalities kindergartens were only established in the 1990s (Jacobsen and Jákupsstova 2005: 170-172).

\subsection{The Åland Islands}

The less populated islands farthest away from Mariehamn, in particular, are facing major challenges, partly in relation to labour supply and partly in relation to demographic changes as these areas suffer from losing their youth and are being left with an ageing population. According to Hovgaard et al. (2004: 31), the Åland Islands have not been through any serious debate about the potential of amalgamations of municipalities, and the municipal structure has been in place since the 1860s (Hovgaard et al. 2004: 27). As is the case in Iceland and the Faroe Islands, the sea is vital to the small local labour markets - on Åland more so with regards to shipping than fishing: "shipping is of great importance not only to the economy of Åland as a whole, but especially to the labour market in rural areas, where possibilities for commuting to Mariehamn are, on the whole, wanting" (Hovgaard et al. 2004: 30).

In Dahlström et al.'s study, they only encountered very few examples of people who commute on a weekly basis from the Ålandic archipelago - and in cases where people did, it was with great costs to their family life and social life (2006: 95). Another approach in order to continue living in the smallest communities is an attempt of combining different types of income and jobs:

\footnotetext{
${ }^{9}$ In addition, to qualify of the perception of the Faroe Islands as a society without significant social divides, Høgnesen (2015) stresses the growing importance of immigration from non-Nordic countries to the Faroe Islands, but also states that very little factual knowledge exists about the possible social divides in this regard.
} 
Across the Åland countryside and archipelago region, local entrepreneurs often combine several activities, such as small-scale farming, fishing or fish farming, tourism, handicrafts, and micro industry. For instance, tourist businesses in the countryside are generally small; many are designed simply to provide additional revenue streams to traditional family farming (Dahlström et al. 2006: 95).

Only people who have a residence permit have the right to own real property or manage a business on Åland - an initiative which, according to Dahlström et al. (2006: 89), has been created in order to ensure that the Åland Islands remain in the hands of the local population. Dahlström et al. (2006) continuously emphasize that in many ways it can be viewed as an advantage that the population of Åland is so small, because it often will pave the way for fast bottom-up initiatives shortening the process from local idea to political and practical implementation.

The strong gender segregation in choice of education on the Åland Islands is echoed within the leisure and cultural activities in that "The girls are more focused on social and cultural activities than the boys, which gives a clear indication of the respective gendered participation in the association activities on Åland. Among the registered association members and active members, women dominate the associations that work with social issues" (ÅSUB 2013: 4, own translation).

A very active association and leisure life, often based on voluntary work, characterize the Åland Islands. In 2011 the 182 registered associations comprised a total of 45,350 members, which equates more than 1.5 association membership per Ålander (ÅSUB 2013: 26). The association life is also quite gender segregated. "Thus, the men are more active in hunting and fishing associations as well as motor and fire brigade associations, but also within sports, while women dominate the more socially oriented associations such as home and school, disability and health-promoting associations" (ÅSUB 2013: 26, own translation). However, some types of associations are also more gender balanced, including arts and craft associations.

Best Practice Case: The Distance Learning Centre in Vágur - Faroe Islands

The Distance Learning Education Centre in Vágur on Suðuroy (the southern-most island among the Faroe Islands) is a "long-distance-learning" centre, which serves as a link between students in remote localities in the Faroe Islands and colleges offering training courses via distance learning. The Distance Learning Centre is an offer for those who want to get an education, whether in the case of higher education, additional training or oth- 
er forms of (further) education while desiring to remain within the community. The Distance Learning Centre provides student guidance in the form of help with the choice of education, and technical guidance on problem solving and project writing. The centre is equipped with classrooms and meeting rooms for students, and a number of technical facilities which the students can use in their distance education.

Vagúr is the second largest village on Suðuroy, and the distance learning initiative is part of a local strategy to attract and retain especially the younger residents of the area, who otherwise may be forced to leave the area if they want an education beyond secondary school. It is especially the women who move to obtain a higher education, which has resulted in a situation where there is a deficit of women in the islands. This gendered imbalance is one of the challenges the initiative wants to address through distance learning. One purpose of the project is to encourage the young people to take all or part of their education locally. So far, the project has been running for almost two years and has been well received.

Besides attracting and retaining younger citizens, the centre is focusing on families, and especially on families in which the husband is working off shore for much of the year, and where it is often the woman who is at home with the family. There are about 100 families in the area with this type of family pattern. Many of these men do not have an academic but a vocational training, often obtained locally. This means that some of them have met their spouse locally, and often they got married and started a family at an early age. The project wishes to facilitate an educational opportunity for these women, as a number of them do not have an education. The Distance Learning Centre has contacted colleges including in Denmark which offers education which these women desire. Especially education programmes within teaching and pedagogics are popular, and at present time the centre has been approached by about 30 women and a few men.

The project has a bottom-up approach, particularly in relation to the education that is offered, and The Distance Learning Centre is in close dialogue with the citizens in the local area through Facebook, to exchange wishes and ideas in relation to courses and training requirements. The project is locally based but also accepts students from other regions of the Faroe Islands. The ambition is to spread the idea of distance learning to more parts of the Faroe Islands and highlight the opportunities that lie in distance learning to remote areas, locally in the Faroe Islands, but also the islands as a whole.

Read more at: 
- http://www.fjarlestur.fo.

\section{Contact person:}

The Mayor in Vagúr Dennis Holm dennis@vagur.fo.

Best Practice Case: Råd \& Dåd - Denmark

Råd \& Dåd (which roughly translates as "Advise and Activity") is a locally based project, started in 1994 in and around the small town of Brovst in Northern Denmark. Råd \& Dåd is a cooperatively owned social enterprise providing jobs on special conditions. Approximately 30 employees are affiliated, who for various reasons cannot hold a job on the ordinary labour market - for instance recipients of social assistance or disability pensioners. Moreover, four supervisors with a professional (often pedagogical) background work at Råd \& Dåd, and these help to guide and coordinate the work. Approximately $1 / 3$ of the employees are women, which according to project manager Rasmus Højen possibly is related to the fact that part of the work is physically demanding. On the other hand, Råd \& Dåd also applies a philosophy of adapting the business according to the people who come - but so far, most people who have applied for a position have been men. The project has, however, focus on attracting both men and women, as well as young and older people, based on an idea that everyone has something to offer, and that diversity helps to create balance.

Råd \& Dåd includes an organic farm shop selling fruit and vegetables from their own nursery. The employees who work in this part of the company are called The Green Team, and besides growing fruit and vegetables and running the farm shop, the team on a weekly basis delivers vegetable boxes to 70 regular customers in the local area. There is also a creative workshop called The Henhouse which produces things that are later sold in Råd \& Dåd's own store of recycled things collected by employees. The Henhouse was originally given its name because there were only women in this workshop, but since then some men also started to work there. Profits from the recycling shop go to charity. There is also a Service Team doing odd jobs - such as lawn mowing, snow removal or minor repairs. The Service Team likes to be seen in the community, and gives priority to those customers who have the fewest resources - such as the elderly who cannot climb up on a ladder or mow a lawn. In the Metal Workshop all sorts of machines are repaired, and local citizens are welcome to show up - this is especially popular among the area's young 
men who can get advice and guidance by the workshop employees for maintenance of equipment. Råd \& Dåd also recently started a Grocery Store in the small village Bonderup. Here the local grocery store closed 7 years ago, but local citizens gathered together to buy shop facilities, and then asked Råd \& Dåd to take over and run the business, which is based in part on voluntary work in weekend and late afternoon shifts, while staff from Råd \& Dåd man the store during daytime opening hours. According to project manager Rasmus Højen, such ideas help to curb emigration and provide vitality in the smaller villages - and very concretely, today fewer "for sale" signs are visible in the streets of Bonderup.

Råd \& Dåd is referred to as an "integral part of" the local community, since it is a project that provides jobs for people who otherwise are outside of the labor market. At the same time, it forges close social ties between volunteers and community members. The project managers and participants are happy to share their experience with this type of business operations, which they believe can be replicated in other communities.

Read more about Råd \& Dåd at:

- http://www.raaddaad.dk

Best Practice Case: E-Forum - Greenland

E-forum was launched by the industry associations in Greenland in 2005 with the support of the Greenlandic Ministry of Church, Culture and Gender. The reason was an increased need to set up a virtual training platform aimed at students and teachers at vocational schools in Greenland, and thus create an infrastructure that would enable fast internet access. Internet access is expensive in Greenland, and therefore the need for a local platform for audio and video access was great. E-forum is currently placed under the Department for Education and Research in Greenland, which is responsible for facilitating this platform, offering courses, programs and Web services.

E-forum, and the digital opportunities the platform provides access to, is central to the development of education in Greenland and takes on special importance in the periphery. Since the project started in 2005, almost all educational institutions (including vocational schools) have been associated with E-forum and make use of their web solutions. The digital platform has enabled education in remote areas of Greenland, which means that people from the villages also have the opportunity to 
attend classes in their local areas. Hence, a digital exam form has become very widespread, made possible through the platform. With E-forum and the platform created under its auspices, Greenland has obtained an opportunity to be in the lead in digital education, which would be an obvious road to follow, due to the large geographical distances between cities and settlements throughout the country.

From the outset, E-forum did not include considerations of gender in their project. However, the project has had an indirect gender dimension, as for instance it has enabled many to take online courses in the evening. This has given especially young mother's the opportunity to get an education, despite the fact of parenthood at a young age, which means that they may otherwise have difficulty accessing education in the daytime. At the same time, women have been quicker to adapt and are more likely than men to move from remote areas and start an education. This has created a certain imbalance in the ratio of boys and girls who take an education in Greenland.

However, it is still the case that the labour market is highly gender segregated in Greenland, and there are no immediate indications that the boys through such online courses in remote areas choose courses in educational fields which have previously been dominated by women and vice versa.

Read more at:

- http://www.eforum.gl/

Best Practice Case: The NITUS-project - Sweden

Nitus (Nätverket för kommunala lärcentra) (The Network for Municipal Learning Centres) is a non-profit association which started in 1996 with the aim of assisting municipalities in jointly promoting higher education and make it accessible to citizens of member municipalities. Today there are about 100 participating municipalities associated with the Nitus network. The creation of advanced studies "at home" was seen as a prerequisite for participation in higher education especially for people such as persons with a vocational training, single parents, students who come from families where there is no tradition for obtaining higher education, as well as persons who for various reasons are not able to move from the local area. This is the starting point for Nitus, and remains one of the guiding principles in the network. 
Work around Nitus has evolved, and today there are increasing demands for Nitus to work on issues around skills development on several levels. Likewise, an extensive exchange of experience in a number of areas takes place within the auspices of the Nitus network, including sharing experiences through workshops, autumn conferences and an annual conference. In addition, knowledge sharing through social media is increasingly gaining importance. To ensure the quality of the various learning centres that are affiliated with Nitus, a number of special procedures and quality assurance criteria have been developed. This quality assurance is recognized by more and more universities, and in many cases it is a prerequisite for membership in Nitus that cooperation between educational centres and universities has been organized.

Through its activities, Nitus has built a network where authorities, organizations and decision makers at all levels are included. The network has been very successful in passing on experience with distance learning from both Swedish and international institutions. Thus, approximately 30,000 students complete a higher education degree through one of Nitus' learning centres each year. Overall, the number of women studying via Nitus learning centres is significantly higher than the number of men. One of Nitus' major challenges and objectives is therefore to encourage more men to study and obtain an education. Another challenge is to stop the emigration from the smaller municipalities, particularly among the young. The Nitus network operates on the belief that to meet the challenges related to gender and population flows, distance learning and learning centres are essential, given that they facilitate the ability to study at university without leaving one's local area. Through this approach, the learning centres (facilitated through the Nitus network) have a decisive impact on the individual student's potential, as well as local municipalities, as Nitus can help stop emigration, while facilitating the training of locals.

Read more at:

- http://www.nitus.se/

\section{Contact person:}

Chairman of Nitus, Kent Wallen, mail: Kent.Wallen@viadidakt.se 



\section{Gender, Work and Working Life in Transition}

In this section, we focus on some of the pervasive changes having occurred in the peripheral areas of the Nordic region and how these changes have a clear gender dimension. The traditional male dominated professions have been particularly affected by these changes, thus particular conditions now apply to the male population of the peripheral areas of the Nordic region. In some of the peripheral areas of the Nordic region, mass unemployment among men is an issue, and some areas seek to adapt to the changing local industry by carrying out retraining projects aimed at these unemployed men finding employment traditionally characterised as "female jobs", such as jobs within the service sector and in nursing.

\subsection{Sweden}

In a study on girls in Småland, Gunnarsson $(1993 ; 1994)$ concludes that many young women in small towns feel that education, business and leisure activities are mainly aimed at young men. While the young men are expected to find occupation within the same industries as their father's, the young women feel more ambivalent about their future. They do not want to live the lives of their mothers. Thus, Gunnarsson concludes that many of the young women might consider finding occupation within the same industry as their mothers; however, most of them will aim at higher posts and full time employment as opposed to their mother's who would often have been content with part-time positions - and part-time positions are now scarce in the peripheral areas.

Johansson, Stenbacka and Nordfeldt (2005) describe how major changes in the Swedish labour market have caused increased regional differentiation. As an example, they refer to Norrland which has an unemployment rate far beyond that of the rest of Sweden; the male population of this area is particularly affected by this:

The forest industry, ore mining and power plants that previously represented the basis for employment in Norrland have lost their significance because of 
technological changes and rationalisation, and businesses belonging to the so-called new economy have not been localised to the northern parts of Sweden. ( ...) It is a well-known phenomenon that areas with poor labour markets will have a surplus of men. One explanation for this is that women are more likely to move away (Johansson, Stenbacka and Nordfeldt 2005: 35, own translation).

Norrland is characterised by its low educational level as regards both the choice of education and occupation. The women who choose to live in the region often have permanent employment in the public sector whereas a large proportion of the men are either unemployed or in short term employments. Moreover, despite several attempts to break with (gender) traditional patterns, the region is characterised by gender segregation. Johansson, Stenbacka and Nordfeldt find that the local conditions affect the men living in the area in different ways, and they identify two strategies: The men either choose to settle for a life with no permanent employment or a long distance commute (permanently or periodically); or they choose to adapt to the altered conditions by obtaining a (gender) non-traditional education such as those within the social care sector. In this context, Johansson, Stenbacka and Nordfeldt conclude that local gender relations and norms are well-integrated but not immutable:

... the men in Norrland must cross the gender boundaries and take on new jobs. These may include doing the dishes, taking care of the children - or working as a fishing guide or social and health care assistant. Gender relations are constantly changing, partly because negotiations and boundarycrossing actions that challenge the old patterns and open up for alternative gender relations occur all the time in everyday life (Johansson, Stenbacka and Nordfeldt 2005: 64, own translation).

According to Johansson, Stenbacka and Nordfeldt tourism is an important industry in Norrland today. However, two issues are highlighted: Tourism is a seasonal industry and some of the local women feel that the activities connected to tourism are too focused on activities often performed by men, such as salmon fishing, scooter safaris and motorcycle tourism. These women believe that the area also needs to focus more on other types of tourism such as cultural tourism. Overall, Johansson, Stenbacka and Nordfeldt conclude that the changes in local labour markets have been so extensive and have occurred over such a short period of time that the individuals, the workers, affected by it have not had time to adjust to the changes. This has a particular impact on the men:

For a long time, being a man in Norrbotten has been synonymous with forestry, hunting and fishing, figuratively speaking. Subsequently, the electron- 
ics industry has been an important employer in Pajala, while the men in Jokkmokk have experienced a more differentiated labour market and have also seen long-term commuting as a solution. Recent developments indicate that a large proportion of the future jobs will be found within the public sector, and in both Pajala and Jokkmokk some men are working within health care. We believe that this will lead to alternative ideas of what it means to be a man in Norrbotten (Johansson, Stenbacka and Nordfeldt 2005: 72, own translation).

\subsection{Norway}

The Norwegian researcher Paulgaard (2002) emphasises how the peripheral areas of Norway have seen changes to local business opportunities. Fishing used to be a very large industry with many workplaces and has remained the largest industry; however, the tourism and travel industries now comprise a new sector. Whereas the fishing industry is centred on the exploitation of natural resources, this new sector provides services; however, Paulgaard points to one similarity between the two sectors - both rely on large national as well as international networks. From a gender perspective, Paulgaard points out that the labour markets of the fishing villages have been highly gender-segregated in terms of the existence of "male jobs" and "female jobs"; however, the new business opportunities have led to the male population of the peripheral areas now working in the service industry in former "female jobs": "That boys and girls are doing the same jobs indicates change, because traditional boundaries between women and men's work are being crossed. But the fact that young people are still working represents continuity with the past" (Paulgaard 2002: 102). When Paulgaard refers to continuity with traditions, she refers to the expectations of young adults to participate in adult culture, thus these fishing villages traditionally have no actual youth culture. As described earlier, Paulgaard (2015) concludes that in some of Norway's peripheral areas school is still 'foreign', a time waster stealing time from young adults who should instead spend their time on what is considered to be "real" work. Though much has changed over time in these areas, Paulgaard concludes that hard work is still considered to form the basis of lifestyle and identity, especially among the male population: "Hard work and being a good "worker" have particularly been an important source of masculine status in such societies" (Paulgaard 2015: 208).

Recently, many of the peripheral areas of Norway which used to have thriving labour markets in fishing, mining or forestry have gone through extensive changes causing a drastic decline in the number of traditional 
(male) jobs. This has created unemployment, especially among the male population (Paulgaard 2006; 2015). In Paulgaard and Bæck's book from 2012 Rural Future?. Finding one's place within changing labour markets, the two Norwegian researchers address some of the issues of their previous research; these are also outlined in this report. Their research addresses issues of gender, education and a changing labour market and work life and how these issues impact young adults as regards their choice of education and mobility patterns.

Angell et al. (2013) emphasise that the Norwegian labour market is still highly gender-segregated, including in the peripheral areas in which most women are employed in the service sector (corresponding to $40 \%$ of women residing in Northern Norway). The men of Northern Norway are, however, mainly employed within building and construction. Angell et al. have also investigated where the workforce of Northern Norway comes from; they emphasise that the employees of workplaces situated in Northern Norway mainly also reside in the peripheral areas; however, since a vast majority of commuters are men, the gender imbalance is particularly evident among employees who commute.

Norwegian researchers Aure (2013a; 2013b and 2011), Fossland (2013a), Grimsrud (2011) and Munkejord (2006; 2011) all focus on immigration in Norway and have made studies among immigrants from various places in and outside Europe on their motivations for and experiences with settling in Norway (especially in the peripheral areas of Norway). Aure particularly emphasises that despite the lack of a highly educated labour force, it may be difficult to find acceptance in the local communities of Norway. Munkejord (2006) also focuses on Norwegians from other parts of the country choosing to reside in Northern Norway and seeks to form an understanding of their motivations behind moving and in particular their motivations behind settling in the peripheral areas as opposed to in the larger cities in the southern part of Norway. Some of the explanations for moving (back) to the peripheral areas of Norway are the opportunities for being close to nature, living close to your workplace, leisure activities, etc.

Aure and Grimsrud have worked on a project on the migration to Norwegian district municipalities solely motivated by the possibility of identifying new job opportunities for both Norwegians from other parts of the country as well as immigrants. In this study, Aure and Grimsrud examined the motivations for settling in the district municipalities among Norwegian and Dutch newcomers, as well as their ability to adapt to the local community and labour market. In their research, Aure and Grimsrud saw a 
cultural difference between Norwegian and Dutch newcomers; the difference was particularly evident in regards to gender relations.

For many Dutch families the good life in the countryside seems to be linked to a stay-at-home mother with young children. Many of the women also want to stay at home in the early years because they believe it will facilitate the integration process for their children. However, the municipalities experience that women who are in employment more easily integrate into the community to which they have moved, and that again means that the whole family might find their place more easily. Therefore, they recommend that immigrant women find jobs as soon as possible. The reason is that women in Norway, including those with small children, are highly active in the labour market, and that employment is a very important integration arena, both for Norwegian and foreign employees (Aure and Grimsrud: 2013, 110-111, own translation).

Aure and Grimsrud emphasise how a number of peripheral areas have designed campaigns for attracting newcomers and thereby for addressing the problem with a declining population in these areas. At the same time, however, they question the points used in some of these campaigns aimed at attracting newcomers to the peripheral areas. The campaigns often focus on the idyllic and quiet settings of the peripheral areas in contrast to life in the larger cities; Moreover, they present a narrative of the good life and how this creates possibilities for living closer together as a family. According to Aure and Grimsrud, the latter might be instrumental in maintaining or even recreating traditional gender roles since some newcomers (most often the women of the family) might need to work less in order for the families to have more time together. "Such messages may be disagreeable to women and men who want a more equal everyday life" (Aure and Grimsrud 2013: 191, own translation).

\subsection{Denmark}

A report from Danish Regions (Tanvig 2010) emphasises that skilled labour is a prerequisite for future growth in the peripheral areas of Denmark. At the same time, globalisation and the internationalised market have created an increased competition from abroad which have put pressure on a number of the traditional industries of the peripheral areas. Furthermore, Danish jobs are increasingly centralised, and with this the demand for mobility among the residents of the peripheral areas have increased. 
Young adults are moving away from the peripheral areas and these areas increasingly experience population ageing. This has implications for the survival of towns and villages; a decreasing birth rate accordingly decreases the need for keeping local institutions, schools, etc. and this leads to institutional closures and a loss of local jobs. This has great impact on the need for the population of the peripheral areas to be increasingly mobile. This not only applies to young adults who increasingly need to complete upper secondary and further education, but also to members of the adult population who need to be increasingly mobile in relation to the labour market (Tanvig 2010).

In his research, Hansen (2011) underlines that, as opposed to young women, young men are closely linked to local work traditions and are thus often inclined to remain in the peripheral areas. However, these jobs are undergoing a period of change, and this creates a number of challenges for the local communities and the men. In some cases this might lead to a sense of frustration among the young men as their expectations for finding employment in their local area may not always be met. As a result, the young men might feel forced to seek employment within an area they might otherwise not be interested in, for the sole purpose of being able to remain in their local community.

Based on his studies, Baagøe Nielsen concludes that the traditional "male jobs" are particularly endangered in the peripheral areas. As a result, the proportion of unemployed men in these areas exceeds that of women. According to Baagøe Nielsen, for the first time in decades, the male unemployment rate exceeds the female unemployment rate, thus creating a new situation. Baagøe Nielsen's research focuses on the declining number of traditional "male jobs", a decline that has led to initiatives aimed at retraining Nordic men for jobs in the social care sector. However, as Baagøe Nielsen underlines, this form of retraining might often collide with the prevailing notion of women as natural caretakers, thus better suited for care-taking jobs. Moreover, few men are attracted to the pay and working conditions of the social care sector, and according to Baagøe Nielsen, this is another key challenge.

In her research on female entrepreneurs, Tanvig (2010) also touches upon the gender segregated labour market. Tanvig argues that a number of factors have affected the regional development of the peripheral areas and thus also the decreasing employment opportunities. One of these is the incompatibility of women's educational choices and local labour markets which causes many women not to return to their local communities upon finishing their studies in the cities, since job opportunities in the peripheral areas do not match their qualifications. This will create a 
future gendered distortion in relation to the population of the peripheral areas. Tanvig believes that sparsely populated areas need to consider alternative business opportunities and suggests that entrepreneurship might be the tool for increasing the female employment rate of the peripheral areas. According to Tanvig, women in peripheral areas most often opt for a certain type of entrepreneurship; a type of entrepreneurship which is highly dependent on the local communities and the opportunities within this community. Thus, women in these areas often start up small businesses with no or only a few employees working part-time or full-time. This might not create many new jobs in the peripheral areas, however, more women might return to the peripheral areas upon having finished their education and remain there to create other forms of employment than those traditionally offered in the local communities; thus these women might be instrumental in evening out the gender balance (Tanvig 2010).

As mentioned earlier, the Danish island Bornholm, situated in the Baltic Sea, is the Danish area farthest away from the rest of the country. Transportation to and from the mainland is either by plane or ferry. In spite of this, the labour market of Bornholm is to some extent connected to the labour market of Copenhagen. In an attempt to accommodate the infrastructural challenges, the island has established several IT solutions, including broadband and workstations at home. Recently, this type of employment has increased on Bornholm, and in 2006 around 1,000 local residents were employed in this type of job. This renders it possible for people to combine living in the picturesque and tranquil peripheral areas with jobs that meet their professional qualifications (Dahlström et al. 2006: 32).

Based on some of the issues related to geographic location and inequalities in the peripheral areas, Dahlström et al. conclude that, much like many other peripheral areas, Bornholm has seen a change in types of employments.

\footnotetext{
Many low-skill jobs in the traditional sectors have disappeared and not been replaced by a similar number of jobs in new sectors. For this reason, among the Danish counties, Bornholm has the highest level of unemployment and a large reserve of labour with a low educational level and professional experience often exclusively from traditional primary or manufacturing sectors qualifications and experiences that are no longer attractive to the labour market (Dahlström et al. 2006: 32).
}

Petersen and Manniche's research on Bornholm as a peripheral area does not include a gender perspective; however, since Bornholm has a large fishing industry, traditionally providing "male jobs", the EU making 
cuts on fishing quotas might have resulted in a decrease in jobs within this industry, and this might have had implications for the men previously employed within this industry who may now be forced to seek other forms of employment.

\title{
4.4 Finland
}

As previously emphasised, the close unity and sense of belonging of North Finland is largely connected to its, at times, harsh nature and a tradition of living in changeable seasons. Old traditions of hunting, fishing and gathering still prevail in peripheral areas of Finland and have profound impact on the labour markets and the population's ability to survive in the peripheral areas. Westman (2005) emphasises that the unemployment rates of peripheral areas of Finland, including Lapland, is particularly high, especially among the male population. Seasonal work is one of the great challenges of the peripheral areas, as it creates a large amount of uncertainty in the local population; however, seasonal employment opportunities are deeply rooted in Finnish traditions and contribute to the creation of a strong sense of belonging.

\begin{abstract}
Seasonal work such as reindeer buck and fishing, are also social happenings that create solidarity and community between people, generations, different ages and genders. It creates the atmosphere of care, independence and cultural community. The interviewees argued that the community and family bonds are stronger in the North than in the South. [...] All interviewees saw that unemployment and short-term contracts are not good for the future of the society. An uncertain future creates family problems, drug problems, problems in paying bills and even poverty. However, poverty cannot paralyse people in Lapland. The nature gives rest and a little bit of food. Gender issues are mainly missing when the question of everyday life and surviving are present (Westman 2005: 134).
\end{abstract}

According to Westman, the highly gender segregated Finnish labour market has great impact on the Finns' work life and the changes this is undergoing today, such as the changes to the work life in Lapland where a vast proportion of the male population is employed within forestry and agriculture, whereas women work within the health and social care sector.

Baagøe Nielsen and Holm (2011) emphasise that the Finnish authorities are tending to the intense gender segregation by having initiated investigations aimed at clarifying the imbalanced distribution of men and women on the Finnish labour market. 
A division of men's work and women's work continues to be the norm in Finland. Desegregation, that is, the division of the labour market based on gender, has been an topical issue in gender equality policy since (the 1980s). Segregation can be seen in men and women largely working within different sectors and in different occupations (Baagøe Nielsen and Holm 2011: 223224, own translation).

Baagøe Nielsen and Holm's work on retraining Nordic men for jobs in the social sector is related to the challenges created by the financial crisis for men in particular - and for their job opportunities. During 20082010, Denmark, Sweden, Norway, Iceland and Finland saw a dramatic increase in the unemployment rate within building and construction. During 2011, however, unemployment rates within this industry experienced a period of stagnation in Finland (and Iceland) (Baagøe Nielsen and Holm 2011).

Baagøe Nielsen and Holm conclude that in Finland, very few men are employed as care workers, especially within child care. Since the next 25 years will see an increasingly ageing population, the need for more men seeking employment within this field will increase accordingly.

The population is ageing. The need for securing the availability of labour within the social care sector is increasing. The need for employing more men in the social care sector is particularly increasing, however, few men seek employment within this sector. Moreover, men are overrepresented among those who fail to finish their studies (Baagøe Nielsen and Holm 2011: 226, own translation)

This increased focus on retraining men for employment within the social care sector is not only a consequence of the ageing population, especially in the peripheral areas of Finland, but also of women increasingly moving away from these areas; moreover, it is an attempt to provide the opportunity for men to seek non seasonal employment.

\subsection{Iceland}

In their report on the Icelandic labour market, to some extent characterised as being isolated in the peripheral areas, Dahlström et al. (2006) provide the following definition of such insular labour markets: when it is not possible to commute in and out of the area on a daily basis. 
Dahlstöm et al. base their report on the Icelandic area, Eyafjörður. ${ }^{10}$ Iceland's second largest city, Akureyri, a city with about 16,450 inhabitants, is located in Eyafjörður; Thus, this area is particularly characteristic of the insular character of the Icelandic labour market(s). Despite its high population, the labour market of Eyafjörður is under massive pressure which is largely caused by geography and accessibility and the four and a half hour drive to Reykjavik (which may be completely inaccessible during heavy snowfalls). According to Dahlström et al., the drastic changes taking place in the labour market and the transition from an industry-based labour market to a knowledge-based labour market place the employees of Eyafjörður, the men in particular, under economic and structural pressure. Though the area has benefited from the growth of the University in Akureyri, which grew from having 4 employees and 50 students in 1987 to having 177 employees and 1,470 students in 2015, this development does not counterbalance the decline in the number of industrial jobs. In their report, Dahlström et al. only touch briefly upon the issue of gender; however, given that industrial jobs are traditionally employed by men, this might have created a higher increase in unemployment among the area's male population. Moreover, the growing tendency of the larger companies of Reykjavik buying businesses in the area is an increasing problem, since the local labour market is already narrow and, as previously mentioned, commuting in and out of the area is simply not possible. This development has also had a negative impact on the possibilities for students graduating from the University in Akureyri to find relevant employment; graduates who might then choose to leave the area to search for relevant employment elsewhere.

The labour market in Iceland is highly gender-segregated (Júlíusdóttir et al. 2013; Rafnsdóttir and Styrkársdóttir 2009: 175), and while female employees are dominant in the public sector, male employees are dominant in the private sector, particularly in the primary sector (agriculture and fishing) and within the manufacturing industry. According to Aðalsteinsdóttir this is largely a result of a vast proportion of Icelanders making gendered education and career choices by excluding certain careers as being incompatible with their gender. More Icelandic women choose traditional male jobs, however, very few Icelandic men choose

\footnotetext{
10 Over time, Eyafjörður has been exposed to much political attention, and in the period 2002-2005 it was selected as a specific focus area in Icelandic regional politics (Dahlström et al. 2006: 41). As a part of this process, some public institutions were moved from the metropolitan area and placed in Akureyri, including "The Office for Wildlife Management" and "the Centre for Gender Equality" (Dahlström et al. 2006: 42).
} 
traditional female jobs (Aðalsteinsdóttir 2010: 44). As described by Rafnsdóttir and Styrkársdóttir:

\begin{abstract}
It is widely believed that Iceland, like the other Nordic countries, has come a long way in terms of equality between women and men. Women's labour market participation is high and, despite a highly gender segregated labour market, women have broken down many walls within "male" jobs, though men have not to a similar extent sought employment in "female" jobs. More women study at universities than men and young people do not feel the need to address equality between the sexes (Rafnsdóttir and Styrkársdóttir 2009: 175 , own translation).
\end{abstract}

Gislason (2011) notes that despite having presented an annual equality plan since 1993, the Icelandic Alping (parliament) has never included the recruitment of men in "female jobs" (such as those within the social care sector) as one of its focus areas. Instead, these plans include specific initiatives towards the exact opposite: increasing the recruitment of women to "male jobs" (such as jobs within the police force). The proportion of male students on the nursing education is between $0 \%$ and $2 \%$ for each year group (Gislason 2011: 240-241). In 2001-2003, the nursing education launched a campaign in Iceland which had only little effect, and educations in pedagogy have not been successful in attracting male students either. Gislason blames the unsuccessful attempts to change the educational choices of Icelandic men on the fact that prior to the economic crisis in 2008, Iceland had no considerable unemployment. Some jobs, such as those within health care, are now considered to be more "secure" and less vulnerable to cyclical fluctuations; Gislason therefore believes, that the crisis might be instrumental in breaking with the traditional gender segregation of the Icelandic labour market.

In Iceland, and the rest of the Nordic countries, far more women than men are part time employed, but unlike the rest of the Nordic countries, the proportion of part time employed women has decreased since 2000 (Haagensen 2014: 88). The Icelandic labour market is also characterised by unequal pay, and this is related to place: Icelandic women are paid less than Icelandic men, and this applies to married women in particular (according to figures from Centre for Gender Equality, 2014) (see also Rafnsdóttir and Omarsdóttir 2010). According to Júlíusdóttir et al. (2013: 266), the largest gender pay gaps are found in the countryside and the peripheral areas of Iceland where men earn on average $38 \%$ more than women. In the metropolitan area average male earnings are 10\% higher than those of women (Júlíusdóttir et al. 2013: 270; and Centre for Gender Equality Iceland 2012: 16). 
Bjarnason and Thorlindsson (2006) draw a parallel between the gender segregation of the Icelandic labour market and the gender pay gap and the differences between centre and periphery:

The most prestigious and highly paid jobs in fishing villages are the traditionally male jobs of fishermen, while the occupational opportunities of women are for most parts limited to low-level service occupations or exhausting, poorly paid jobs in local fish-processing factories. As most farms in Iceland are small-scale family farms, the gendered division of labour is less visible in governmental statistics. Nevertheless, research has shown that in addition to their participation in indoor and outdoor farmwork, many farmwives must work long hours in low-income jobs outside the farm to supplement the income of the family (Bjarnason og Thorlindsson 2006: 292).

Rafnsdóttir and Ómarsdóttir (2010) explain how, in the wake of the economic crisis in the autumn of 2008, the Icelandic government appointed a working group, Equal Rights Monitoring, for the purpose of "assessing how the crisis affected gender equality, and monitoring whether and how the economic crisis affected women and men respectively" (Rafnsdóttir and Ómarsdóttir 2010: 89, own translation). Later analyses conclude that the crisis did in fact have different impacts on men and women. The Centre for Gender Equality Iceland elaborates on this:

The number of men in the workforce has decreased more substantially than the number of women. In 2008 men's job participation was $87.1 \%$, but in 2010 it had become $84.5 \%$. In comparison, women's participation went from $77.8 \%$ in 2008, down to $77.6 \%$ in 2010" (Centre for Gender Equality Iceland 2012: 14).

According to a study conducted by Karlsdóttir and Ingólfsdóttir (2011), Icelandic women, especially those living in small rural communities, have often been forced to exercise a great deal of flexibility in order to create a place for themselves in the labour market. Whether caused by necessity or desire, the increased flexibility of women is also evident in the increased proportion of women starting up their own businesses. In 1990, 11\% of all new business owners were women, in 1998 this increased to $17 \%$ and in 2008, $21 \%$ of new business owners were women (Rafnsdóttir and Styrkársdóttir 2009: 168).

Thus, the Icelandic labour market is characterised by two significant distinctions: the gender imbalance across the different sectors and the significant differences in the labour markets of the metropolitan area and the remaining regions. Across these differences, Júlíusdóttir et al. emphasise the existence of a highly ethnicised labour market in which certain types of jobs are only available to either men or women, resi- 
dents of the capital or of the countryside, immigrants or Icelanders and even women or men of ethnic backgrounds. In total, the service sector constitutes a far greater proportion of the labour market of the metropolitan area, gender segregation is particularly widespread in the countryside and immigrant workers are clearly overrepresented in the processing industry - particularly in the part of the industry located outside the metropolitan area (Júlíusdóttir et al. 2013: 271).

The primary sector accounts for $5.9 \%$ of Icelandic employment; this is the highest proportion in any of the Nordic countries and this may be attributed to Iceland's fishing industry. Thus, the export of fish constitutes 39\% of Iceland's total export earnings (Haagensen 2014: 133). Edvardsdóttir (2013) emphasises how the business life of small Icelandic communities are particularly unified and highly dominated by the fishing industry which is a profession highly dominated by men. Thus, she writes:

All around the world the fishing industry is male dominated; women don't fish, they don't run the fishing companies and they don't sit on the company's boards. They don't see themselves as fishermen although they take part in the fishing process, e.g. baiting the line, working in the fish plant or doing the book-keeping. Even though they were doing things that can be called managing the business, they don't see themselves as managers or that they are in a position of having power. In accepting the status quo people rationalise the absence of gender in an occupation. Then it is common to use arguments that are linked to intrinsic abilities of masculinity and femininity (Edvardsdóttir 2013: 84).

In recent years, the fishing industry has been subject to rationalisation caused by cyclical fluctuations and the introduction of fishing quotas. According to Rafnsdóttir and Omarsdóttir the decline of the fishing industry have "had a particular impact on traditional female jobs in the regions when efficiency improvements and declining fish stocks have forced fishing factories to shut down" (Rafnsdóttir and Omarsdóttir 2010: 71 with reference to Skaptadóttir and Proppé 2005, own translation). Furthermore, they argue that regional labour market policies have often focused on promoting employment within traditional male jobs. Moreover, the changing fishing regulations have generated a shift of power between local men; Bærenholdt (2007) uses the terms quota kings or sea lords in reference to the struggles between male fishermen following a group of Icelandic fishermen having purchased fishing quotes in an effort to control the market.

According to Júlíusdóttir et al. (2013), the shifts of the Icelandic labour market are closely related to issues of gender and place. First of all, they comment on how a vast amount of Icelandic women left the fishing 
industry during the 1990s. Moreover, more and more Icelandic women opted for education and new employment opportunities, primarily within the service sector, which in turn led to an increased amount of women leaving the small communities:

\footnotetext{
The increased education of women was an important factor [...] Equipped with higher education and new values, women in fishing towns turned their back on fish processing, preferring public-sector and private-sector service jobs. The increase in public work in care and education at municipality level provided new job opportunities for women. Many women left the smaller fishing towns for a more diversified labour market and career opportunities in the capital region and regional centres (Júlíusdóttir et al. 2013: 268).
}

The Icelandic women having left the fishing industry were largely replaced by migrant workers, especially women from Poland, Thailand and the Philippines who would often marry Icelandic men and settle permanently in the Icelandic community (Skaptadóttir 2015). ${ }^{11}$ During the restructuring of the Icelandic fishing industry, the organisation of work within agriculture and tourism went through similar changes; agriculture changed its focus to horse breading while tourism focused on horseback riding tours on Icelandic horses. This lead to an increased number of foreigners (mainly young women from Scandinavia and Northern Europe) working seasonal jobs in the countryside. In their study of women having chosen to remain in the small community Húsavík, Karlsdóttir and Ingólfsdóttir (2011) emphasise that many of the women who were willing to adapt to the changing labour market have found employment in the tourist industry.

Moreover, Júlíusdóttir et al. (2013) conclude that the Icelandic labour market has also seen an increased number of foreigners, primarily men, being employed as a result of the fast growing construction industry (of 2005-2008). These men were recruited in countries such as Poland, Portugal and China partly to work on construction projects in Reykjavik and on megaprojects, including hydroelectric plants and aluminium factories in the peripheral areas. The economic collapse of 2008 was a turning point which put an end to this recruitment and caused both Icelandic men and women to emigrate in an attempt to find work.

\footnotetext{
${ }^{11}$ As a result of Iceland's entry into the Schengen Agreement in 2000, the possibilities for non-EU citizens to immigrate to Iceland were limited, and the chain migration practice among migrants from the Philippines were brought to a halt (Skaptadóttir 2015 and Júlíusdóttir et al. 2013). The majority of migrant workers in Iceland and women in particular - are overqualified for the jobs they perform (Júlíusdóttir et al. 2013: 270).
} 
The great changes following the financial crisis are also evident in the migratory pattern of the Icelandic population. Thus, Júlíusdóttir et al. describe that before 2009, there "were no significant gender differences in the emigration of Icelandic citizens or their return migration, which was high" (Júlíusdóttir et al. 2013: 271). At present, however, more Icelandic women choose to leave Iceland compared to their male counterparts, and the emigrants seem less inclined to return to Iceland than previously. Júlíusdóttir et al. ascribe this changing pattern to a combination of the better employment and income opportunities and lower housing costs of overseas countries.

In conclusion, however, Júlíusdóttir et al. state that most Icelandic areas do not see a distinctive proportion of women leaving the areas outside the metropolitan area, and this "is counter to the long-observed trend in Iceland and other peripheral areas in the Nordic countries that women, especially young women, dominate outmigration" (Júlíusdóttir et al. 2013: 267). Júlíusdóttir et al. refer to immigration to Iceland from abroad as a possible explanation for this and as a significant factor in preventing a general decline in population during this period. At the same time, the north eastern region is the only region experiencing a net immigration of Icelandic women during this period. According to Júlíusdóttir et al. this might be explained by the fact that "(m)ost of the aforementioned women move to Akureyri, the regional centre in the North, which has both a university and a regional hospital" and in general "(a) relatively high rate of in-migration of women occurs in the few places outside the capital region offering tertiary level education" (Júlíusdóttir et al. 2013: 267).

\subsection{Greenland}

The labour market in Greenland is highly gender-segregated. In 2002, out of the 12,000 employed women in Greenland, 8,000 of these were employed in the public sector; however, the private sector is largely dominated by male employees (Eistrup and Kahlig 2005: 211). In general, the employment of women in the West Nordic regions is "higher than anywhere else in the Nordic countries, however, the pay gap between men and women appears larger in the West Nordic regions than in the other Nordic countries" (Rafndóttir 2010: 12, own translation). In $2006,44 \%$ of those engaged in active employment (measured in fulltime equivalents) were women and the majority of public sector employees were women; thus, in 2006, the amount of female public service 
employees were nearly twice that of men (Poppel 2010: 39). At 9.8\% in 2012, Greenland's unemployment rate was the highest among the Nordic countries. Unemployment was particularly high among young adults, with small variations across gender (Haagensen 2014: 84).

Eistrup and Kahlig 2005 argue that to some extent, Greenland's current patterns of gender segregation related to educational choices and labour market affiliation may be an offshoot of the colonial period. In their review on the gender relations of modern Greenland, Eistrup and Kahlig conclude that the modernisation process of Greenland has significantly altered the relations between the genders. The Danish colonial rule aimed at creating a wage-earner mentality, and according to Eistrup and Kahlig, this entailed two characteristic changes to gender relations: 1 ) since Greenland's women were in paid employment in the fishing industry while Greenland's men were fishermen, the female population was regarded as crucial to the modernisation policy; 2) the relationship between young female Greenlandic wage earners and young male Danish public servants and workmen affected the gender relations in Greenland. According to Eistrup and Kahlig, the Greenlandic men became the losers (Eistrup and Kahlig 2005: 204, with reference to Arnfred 1991: 105).

Poppel argues that the development of recent years have created major changes in gender relations in Greenland, partly because of educated and employed Greenlandic women no longer being as financially dependent on their husbands or partners as they have been in the past. Today, we might even argue, "women are increasingly becoming the main providers" (Poppel 2010: 55, own translation). Rasmussen (2009) further suggests that even successful fishermen and hunters in practice often rely on their spouses' income. Thus, a growing number of households depend on income from the women, and more than half of the homes where hunting and fishing represent a significant part of the revenues, it is women who earn most of the money. As Rasmussen states: "Single men without these income sources, however, are confronted with severe economic problems" (Rasmussen 2009: 526), and an increasing number of these may in practice often rely on living as "couch surfers" with family and friends, or receive help from ageing parents, which according to Rasmussen only amplifies the extent of social problems in the small villages.

Despite their growing labour market presence and increased educational level, in medium or higher educations in particular, Greenland's women are underrepresented in managerial positions and have lower average earnings than Greenlandic men. However, according to Poppel, this pay gap has substantial local variations; the pay gap in the cities is far greater than in small villages (men on average earn 39\% more than 
women in the cities and in the small villages the number is "only" 26\%) (own calculations based on numbers from Poppel 2010: 63).

According to Weyhe (2011), the financial crisis of 2008 is not as threatening to the traditional male jobs as is the case in many other Nordic countries: "Admittedly, traditional Greenlandic male jobs such as whaling, seal hunting and fishing are at risk; however, the growing industries of the future are predicted to create many new employment opportunities within (these) traditional male jobs" (Weyhe 2011: 248 ,own translation). Here, the challenges primarily extend to the availability of skilled labour which we will look into later. Furthermore, Weyhe emphasises that Greenland has not been as affected by the financial crisis as the rest of the Nordic countries (Weyhe 2011: 249). In conclusion, Weyhe claims that the financial crisis in Greenland has "not created structural changes to the labour market which might motivate men into changing their professions across the gender-segregated labour market. Admittedly, a number of traditional male jobs are affected by the financial crisis, however, current and future male jobs will still be in need of skilled labour" (Weyhe 2011: 257,own translation).

Thus, it is hard to envisage that the current structures characterising Greenland's labour market will undergo changes in the near future. Despite the shortage of labour within specific parts of Greenland's public sector, including the teaching profession, it is hard to detect any incentives for men to opt for "female jobs". Weyhe concludes on this by emphasising that this does not mean, "however, that the need for focusing on gender and diversity on Greenland's labour market is non-existent [...] Challenging the gender stereotypes preventing individuals from moving freely across the gender segregated labour market remains essential" (Weyhe 2011: 258-259,own translation). Weyhe places particular emphasis on Greenland's fishing industry as an industry in which "gender, work, lifestyle and geographic location are often closely connected and this group might thus be particularly vulnerable to processes of change" (Weyhe 2011: 249,own translation); moreover, he points to the non-existent knowledge about the willingness and ability to complete any retraining or upgrading of skills among these men.

A widespread willingness and interest in upgrading within traditional professions is, however, one of the major conclusions of an analysis of the hunting profession in Greenland today (Rasmussen 2005). But precisely upgrading in relation to traditional occupations, and a more limited interest in outright retraining. The recommendation in Rasmussen s report was the establishment of a fisheries and hunting school that could provide young people the opportunity to gain insight and qualifications in new 
technologies in fishing and hunting, which could ensure an influx of young people to these professions. These traditional activities still play a role especially for young men, because it gives them some income opportunities in their communities that would otherwise not available. However, another publication suggests that, unlike Greenland's men, women are far more inclined to indicate the possibility of obtaining further training or education as a reason for wanting to move to a different area (Nordregio 2010: 133). Hence, for some women, education seems to be part of a strategy to relocate elsewhere. The report Mobility in Greenland (Nordregio 2010) points to rather significant gender differences in Greenland's mobility patterns and ascribes these differences to two factors:

The first of which is the expectations to the contents of a job and to social relations, and the second factor is the matter of education and training and of employing qualifications. Women particularly value having a challenging job that holds opportunities for both professional and social development. By contrast, men place greater emphasis on salary (Nordregio 2010: 66, own translation).

Thus, this study clearly indicates gendered differences, some of which are considered essential for the decision of moving to a different area. A large proportion of men indicate that the matters of career and job quality are essential reasons for moving, while women are more inclined to indicate family, children and leisure activities as their key priorities when deciding to move. Moreover, the study shows that women are more inclined than men to indicate "partner's job" as a reason for moving. The report highlights this imbalance as a possible demonstration of the absence in gender equality in family relationships, but also that this imbalance is undergoing change (Nordregio 2010).

The report points to significant gender differences regarding which particular industries the respondents find attractive, however, the report also indicates tendencies not relating to gender; the population in the small villages prefer traditional jobs (whaling, seal hunting, fishing and the extraction of raw materials) while the urban population are more oriented towards the service sector. Furthermore, the report identifies a possible and quite interesting change in attitudes which might eventually counteract the gender segregation of labour markets:

The difference in the responses given by younger men in small villages and cities indicates that these social boundaries might not be eternally lasting. In contrast to men living in small villages, far more men living in cities show an interest in jobs in health, social institutions and teaching. This difference both reflects a difference in attitudes and a difference in which types of jobs are de facto available in these different areas (Nordregio 2010: 19,own translation). 
In terms of new business opportunities, for a number of years, Greenland has increased its focus on the potential demographic impact of new projects proposed within large scale extraction of raw materials and natural resources. As indicated above, Nordregio's report concludes that more men than women show an interest in jobs relating to any future large scale projects on the extraction of raw materials; moreover, the interest among the rural population exceeds that of the urban population. The debate on the possibilities for undertaking large scale productions of raw materials is vital since these projects might show that, in addition to the financial effects, they might eventually "contribute to significantly restructuring Greenland's society", as is stated in the report (Nordregio 2010: 11).

This restructuring not only relates to the utilisation and further qualification of the labour force, but also to new business opportunities, such as those within the oil and mining industries, and how they affect local communities. Some of the key issues in this context is whether workers, predominantly men, are willing to settle permanently in the areas in which jobs are created or whether this will result in the emergence of new mobility patterns based on the rhythms of work of industries; as an example, the offshore industry typically follows a pattern of two weeks of offshore work followed by a two week break (Pristed Nielsen 2015, in print). By creating opportunities for workers to earn a salary while maintaining a connection to the local community, new activities in the extraction of raw materials might contribute to improving the local economy and strengthening the local community. When workers choose to settle permanently in areas presenting new income opportunities, whether or not they bring their families is essential to the future development of the area, since this will not only have consequences for the social relationships within the family but also for local communities and, on a structural level, for the supply of schools, day care, etc. Against this background, Weyhe stresses the need for taking gender specific assessments into account when planning major business projects. This might contribute to the possibility for recruiting more women to traditional male jobs as a strategy for creating cohesion in local communities by providing employment for both husbands and wives - a necessity when keeping the current housing situation in Greenland in mind, as this renders double housekeeping impossible (Weyhe 2011: 253). 


\subsection{The Faroe Islands}

According to Gaini (Gaini 2005), modernisation and industrialisation did not reach the Faroe Islands until very late; not until the end of the 19th century. Modern market economy and the introduction of the Nordic welfare system did not reach the Faroe Islands until the industrialisation of the fishing industry in the 20th century (Gaini 2011a: 151 with reference to Raoulx 1992). Jacobsen and Jákupsstova describe how particularly the increased export within the fishing industry led to the Faroe Islands experiencing "a number of radical financial, social and cultural changes leading to a more open and dynamic fishing community" (Jacobsen and Jákupsstova 2005: 185, own translation). Moreover, they state that the monetary economy following this development helped form a male ideal of entrepreneurs which regarded women as those responsible for family life and for supporting their husbands while they developed new companies (Jacobsen and Jákupsstova 2005: 170).

According to Knudsen (Knudsen 2009), the fishing industry is most often regarded the basis of the economy of the Faroe Islands, and fishing also constitutes $95 \%$ of the total export earnings of the Faroe Islands (Gaini 2005: 14). Knudsen writes the following on fishing and gender:

Most of the political and public debate is focused on fishing, and it has been (and is still) male-dominated. The role of women (since the establishment of many of the fish fillet factories in 1960) was intended to be a working capacity characterised by flexibility and the willingness to accept irregular jobs depending on the supply of raw materials (Knudsen 2009: 333, with reference to Jacobsen 2007: 211).

At the beginning of the 1990s, a deep economic depression affected the Faroe Islands and large parts of the fishing industry went bankrupt, the unemployment increased, particularly among men and more than $10 \%$ of the population emigrated - mainly to Denmark (Gaini 2005: 14; Jacobsen and Jákupsstova 2005: 172). According to Gaini (Gaini 2005), the extent of this crisis was unparalleled in Europe and it radically changed the Faroese society.

Overall, the business community of the Faroe Islands has always been connected to marine resources. Nevertheless, a relatively small proportion of the labour force is actually employed in the fishing industry, whereas a large proportion of the labour force is employed in the service sector, much like the rest of the Nordic countries. Like the rest of the Nordic countries, a vast proportion of those employed in the service sector are women. The female part of the Faroese labour market is very narrow and Faroese 
women are primarily employed within the public sector and trade, whereas Faroese men are spread more evenly across different industries, but constitute the vast majority of people employed in fishing, agriculture, production and building and construction (Knudsen 2009: 335). As concluded by Hovgaard (2015), the public sector of the Faroe Islands is important to the gender balance and to the career opportunities of Faroese women. According to Hovgaard, when Faroese men working as Long Distance Workers in the maritime sector choose to have their permanent address on the Faroe Islands, this is often motivated by providing the opportunity for their wives to find local employment. Hovgaard states that long distance work, as a profession and method of working, is in rapid growth in the Faroe Islands; and this method of working may potentially contribute to the future desire for maintaining a well-functioning local community on the Faroe Islands. However, this type of job will undoubtedly appear more attractive to men than to women, and Hovgaard discusses the implications of this in his article.

Literature on the subject points to a vast difference between the average earnings of men and women in the Faroese labour market. Knudsen comments on this: "The Faroese women's overall level of income is significantly lower than that of the Faroese men despite Faroese women having the highest employment rate of the Nordic countries" (Knudsen 2009: 331 with reference to Agerskov 2008: 121, own translation). ${ }^{12}$ Generally speaking, this pay gap has remained unchanged for the past 25 years (Knudsen 2010: 31), and the large pay gap between men and women even gave rise to an annotation in the report on the Faroe Islands completed by the CEDAW Committee in 2006 (Knudsen 2009: 325).

In part, the pay gap may be explained by the large number of part time employments on the Faroe Islands, particularly among women. "In the Faroe Islands, 56 per cent of the working women work part-time" (Haagensen 2014: 88). The large pay gap, however, cannot be explained solely by the large number of part time employments. Overall, Knudsen ascribes structural inequalities as a contributory factor to the large pay gap between men and women. She highlights the company Faroe Seafood which, in 2009, consisted of 900 employees, a vast majority of whom were women. However, in 2009, none of the five members of the company's top management were women (Knudsen 2009: 336; 338).

12 In 2008 Faroese men thus earned $62 \%$ of the total wage payment, while the figure for Faroese women was 38\% (Knudsen 2010: 30). 
Moreover, the high salaries of the male-dominated fishing industry, which are often higher than the salaries of academics, might explain the large average pay gap between men and women in the Faroe Islands (Gaini 2005: 17). Thus, Gaini states that young Faroese adults, especially young adults from small villages, often make a rational economic decision when choosing to work within the fishing industry; whether in the form of a spare time job or a holiday job. Young adults traditionally take a job after school; this may be a regular employment or they might just help out in the family business (Gaini 2005: 18). Gaini emphasises that the concept of "youth" is fairly new in the Faroe Islands: previously, young boys in particular would start working with their father's and/or uncles as early as from the age of $12-13$, thus, they would go straight from childhood to adulthood (Gaini 2006: 46).

In relation to the extent and speed of which the changes of the Faroese labour market have occurred, Gaini (2005) takes the interesting perspective that today's work life and the need for focusing on adaptability and flexibility, not least created by processes brought about by globalisation, is actually not very far from the traditional structure of Faroese work life. Traditionally, large parts of the Faroese population have lived in small communities, and this has created the need for the local population to spread across different branches of the social, financial and political structures of the local community.

\subsection{The Åland Islands}

The Åland Islands have the lowest employment participation rate in the Nordic region; at the same time, it is the only country in the Nordic region where the employment participation rate of women is higher than that of men (Haagensen 2014: 82; ÅSUB 2015: 5). Unemployment is low in the Åland Islands, and Ålandic women hold the Nordic record with only 3.1\% unemployed in 2013 (Haagensen 2014: 84). The low employment participation rate can be explained by the high number of pensioners in the Åland Islands. At the beginning of the new millennium, women's employment rate caught up with that of men and has since then continued to increase (ÅSUB 2014: 12). A report from ÅSUB estimates that "A possible explanation is that women's employment is dominated by the expansive health and care professions in the public sector more so than that of men; at the same time, men's vocational choices have typically been within those parts of the private sector which in recent years have undergone significant rationalisations, not least within shipping" (ÅSUB 2014: 4-5, 
own translation). Since the beginning of the financial crisis in 2008, the difference between men's and women's employment rates has thus increased (ÅSUB 2013: 10). Furthermore, a contributing factor with regards to the employment participation rate is the fact that Ålandic men retire earlier than Ålandic women (ÅSUB 2014: 17).

In addition, the Åland Islands have experienced considerable progress within "female jobs", and the number of people employed in the public sector has grown by over $20 \%$ in the period $2000-2010$. By comparison, during the same period, the growth rate in Finland was $10 \%$, and in Sweden only a few percent. On the other hand, employment levels within the transport and shipping sector have been marked by the opposite trend: an 8\% employment reduction in Åland as compared to a reduction of 3-6\% in Finland and Sweden (ÅSUB 2013: 12).

Similar to many other Nordic countries, Åland predicts to meet challenges in recruiting workers, particularly for the care sector. The total workforce of the Åland Islands is extremely small, and in 2004 it only totalled about 13,000 people (Dahlström et al. 2006: 90). Moreover, the labour market is characterised by not being very dynamic and most employees remain in the same position without changing their job. According to Dahlström et al. (2006), unemployment in the Åland Islands carries a stigma:

"If you didn't have a job, you created one." This quote from one of the interviewees in Åland reflects the strong norms that exist there on the importance of working. To be unemployed was not really an option, thus Ålanders have tried to make sure that they either earn an income through paid employment or by running their own businesses (Dahlström et al. 2006: 89).

Although the primary sector is considerable in the Åland Islands and on a par with Iceland (they share a Nordic first place), the tertiary sector accounts for the vast majority of the total employment; and the Åland Islands also claim a first place here (Haagensen 2014: 87). In other words, the manufacturing industry represents a relatively small part of the Ålandic labour market, while the tertiary sector - shipping in particular - represents a substantial part of the labour market. "The production structure of Åland's economy is dominated by shipping, which accounts for approximately one fourth of those employed in the labour market, while manufacturing accounts for only about $10 \%$ of total employment" (Dahlström et al. 2006: 91).

The dominant shipping industry actually employs more people than can be found in the local labour market, and the sector thus recruits workers from outside the islands; according to figures from ÅSUB, 1600 
foreign workers are employed on Ålandic vessels (2013: 13). With its well-developed public system, the Åland Islands also have a significant public sector, which is of great significance to women's employment in particular. In 2003, 2100 people were employed in the public sector alone, and the sector is estimated to account for more than half of the total employment. Shipping accounts for nearly $20 \%$ of the total employment (Dahlström et al. 2006: 91).

The strong tendency towards women being employed in the public sector, while men are employed in the private sector, causes a pay gap in the short run and a retirement savings gap in the long run (ÅSUB 2015: 13). In general, women's income is lower than that of men - an average of $82 \%$ of men's income (ÅSUB 2012: 4) One explanation is that more women than men are in part-time employment. Thus, the Ålandic women only account for $43 \%$ of the total taxable income in the islands of the 2012 tax year ( $̊$ SUB 2015: 15); however, there is a slight tendency towards bridging this gap (ÅSUB 2012: 17). Moreover, the pay gap is smaller in the younger age groups. Nonetheless, women employed in the private sector continue to have a lower starting salary than men, but in the public sector the starting salary is more or less the same for both genders. However, since the pay gap in the public sector increases with age, the result is that the total pay gap is roughly the same size in both sectors (ÅSUB 2015: 16). In spite of the fact that there are more female than male pensioners, the total income of female pensioners is lower than that of men (ÅSUB 2014: 5). In 2011, women's average pension was 1,220 Euro per month as compared with 1,744 Euro for men (ÅSUB 2013: 23). A more detailed review of the pay gap measured on the basis of different principles and parameters may be found in the ÅSUB report (2013: 21).

The choices made by young adults as regards to education and career are largely similar to that of their parents' generation, and "therefore, most indicators suggest that the clear gender differences in the choice of employers, industry and field of work - and thus long-term career and income opportunities - are unlikely to change much in the foreseeable future" (ÅSUB 2015: 14, own translation). The gender segregation in the Ålandic labour market may be illustrated by drawing a list of the ten most popular types of jobs among men and women respectively: only three job types appear on both lists, namely in the restaurant business, sales and the insurance and financial sector (ÅSUB 2013: 4; 16). All other professional groups are strongly gender segregated; and there are no apparent signs of changes (ÅSUB 2013: 4). On the other hand, there is a slight tendency that the pay gap between men and women will be nar- 
rowed, particularly if the differences in hourly earnings are viewed separately, which will neutralise from the statistics the fact that many women are employed on a part-time basis (ÅSUB 2013: 5).

In addition to the two major sectors, the shipping and the public sector which are dominated by men and women respectively, the Åland labour market is characterised by having many small businesses. These businesses are founded by both men and women:

(M)icro businesses dominate the economy of Åland. The typical firm being a family business with just a couple of employees, busy within different activities, or even within different industries. When it comes to business start-ups, most new enterprises are to be found within the social and personal services sectors, and in trade, hotels, and restaurants, or in the construction sector (Dahlström et al. 2006: 93).

This is confirmed in a report from ÅSUB, which argues that "The Åland business structure is greatly dominated by a large number of small businesses. Around 88 percent of all Åland businesses have less than five employees, whereas less than one percent of all businesses have 100 or more employees. [...] The typical Åland business is a small family business with a few employees" (ÅSUB 2008:17). In fact, it appears from one figure in the same report that in 2008 only three business in all of Åland had more than 250 employees (ÅSUB 2008:18).

In 2008, ÅSUB carried out a major study to map out "women's entrepreneurship and business involvements in Åland, with the aim of clarifying motivational factors, motives and obstacles" (ÅSUB 2008: 3). The political objective behind the study was the diversification of the Åland labour market (ÅSUB 2008: 12). The study was based on available statistical material as well as on a survey conducted among 240 small businesses and in-depth interviews with 17 female entrepreneurs. Åland has a high rate of newly established businesses; approximately 200 businesses are established each year, but as a number of businesses also close down, the increase rate is slightly lower (ÅSUB 2008:19). In 2005, women accounted for $31.6 \%$ of all self-employed individuals in Åland, against 25\% in Sweden and 33\% in Finland. However, the share of women entrepreneurs in Åland seems to be fairly constant, whereas shares are increasing in the neighbouring countries (ÅSUB 2008: 8). Salary payments in these businesses have seen a steep growth, with an average of $10.3 \%$ per year from 2000 onwards, against $3.7 \%$ in the labour market in general ( $̊$ SUB 2008: 33). Hence, profitability is fairly high in these businesses, but still lower than in large parts of the labour market in general. 
Although entrepreneurship is highlighted as a phenomenon which might be of particular significance to the areas outside Mariehamn - in the small towns and in the archipelago - the report merely seeks to illustrate the conditions of these areas by mentioning 1) that historically, particular primary professions such as agriculture have had significant impact on employment rates outside Mariehamn (ÅSUB 2008: 16), 2) that small independent companies have been relatively more important to the labour supply outside Mariehamn (ÅSUB 2008: 30), and 3) that the geographical location of companies are more important to young female entrepreneurs - without providing any further explanations as to the reasons for this (ÅSUB 2008: 65). The overall statement made, however, is that:

...in the Archipelago, it is more common to be self-employed, whereas in Mariehamn, it is more common to be employed. When only considering selfemployment, the women's share in this is highest in Mariehamn and lowest in the villages of Åland [...], which may be explained by occupational structures. The primary occupations are dominant in the villages, whereas service jobs are more common in Mariehamn (ÅSUB 2008: 30, own translation).

As women tend to start their own businesses within other areas than men, entrepreneurship in general does not tend to be a phenomenon contributing to a reduction in gender segregation in the Åland labour market - quite the contrary. "As regards entrepreneurship, segregation between different lines of business does seem to be even higher than in the labour market in general" (ÅSUB 2008: 29).

In conclusion, Dahlström et al. (2006: 99) state that the Åland labour market contains a number of opportunities and challenges in regards to its future development. Some of the most significant aspects relating to the focus of this report are, on the plus side: The geographical location of the Åland Islands between the two rapidly growing markets of Stockholm and Helsinki, fast and easy access to public authorities and a strong tradition for entrepreneurship and micro enterprises. The more challenging aspects relate to: a fragmented internal labour market caused by the uneven population development concentrated around Mariehamn, the difficulties of persuading young adults to return to the islands upon finishing their studies and a growing tendency among young adults of being more hesitant to start up their own businesses than older generations.

Best Practice Case: Project Qualifying for New Jobs - Denmark 
"Qualifying for New Jobs" is a project under the auspices of AMU (Labour Market Education Centre) North Denmark, which was launched in 2011/2012 and is running until the summer of 2015 (after an exceptional extension). At the time of completion, the project will have had about 440 unemployed participants. The project is a Social Fund Project, which consists of training courses which are tailored to each participant and aims to transform unskilled long-term unemployed to qualify for skilled jobs. The project has had a special focus on growth/job potential in all parts of North Denmark - and has been geographically spread across four local units in the region, to ensure that the project was locally based.

The individually tailored programs in the project are planned so as to ensure the best possible match between the individual job requirements, and the need for qualified labour in the Region of North Denmark. Prior to the project start, there was a clear expectation that there would be a majority of male participants. This assumption was based on the observation that by the onset of the project in 2011/2012 there was a situation in which many unskilled men had to find new (types of) jobs due to the financial crisis. It has turned out in the process, however, that women's unemployment increased, which has led to a gradual increase in the number of women on the teams. This means that the initial expectation that most men would take up the offer has been replaced by an even gender distribution, so that according to self-assessments of the project there is a $50 \%$ distribution between the sexes.

The methodology of the project is focused on individual guidance and intensive dialogue, which has given good results. The perception among the initiators of the project is that there is a lack of offers for adults comparable to what youth education counsellors provide to young people, and that this project has been able to provide the needed guidance and support. The project has demonstrated that thorough documentation of participants' prior experience may lead to a formal recognition in the form of education/certificates of competence, which could shorten the training time, if for example a vocational training is pursued - perhaps completing such an education whilst working. The overall project goal has been that the individual unemployed person reinforces his or her professional and personal flexibility, and achieves a stable attachment to the labour market.

Work is currently underway to create an extension of the project, which will target the unskilled people in North Denmark who have the potential to become skilled in a shorter time than the norm. That is, based on credit for previous job experience/training, etc. The extended version 
of the programme will be directed towards both employees (about 900) and unemployed (about 400) and is thus an ambitious project, which will involve both education-, employment- and business efforts.

Read more at:

- http://www.kompetencenord.dk

\section{Contact person:}

Project leader Dorte Sif Boddum Kronborg - mail: dbk@amunordjylland.dk

Best Practice Case: Brautargengi - Project for female entrepreneurs Iceland

Brautargengi ("Prosperity") is an initiative that supports and guides female entrepreneurship in Iceland. The initiative was launched in 1996 in Reykjavik during a period where unemployment among women was very high compared to men. To meet this challenge, the city of Reykjavik supported the project and helped to co-finance the course. Akureyri (the second largest city in Iceland) and a number of peripheral areas launched their version of the Brautargengi project in 2002, and until the financial crisis in 2010, the various municipalities which participated in the project contributed with financial support. The participation fee for people in remote areas is a bit less than in the metropolitan area, which is closely linked to demand in the two areas.

Due to the economic crisis, the city of Reykjavik withdrew its support for the project in 2010. Despite the lack of financial support from Reykjavik, the project is still ongoing, which is due to the huge success of the project. The course is now entirely run and managed by Innovation Center Iceland, both in the capital, but also in various remote areas in Iceland. More than 988 women have completed the course, and an independent survey from 2010 indicates that the share of start-ups which are initiated by women who have completed the course is just as high in Reykjavik as in various peripheral areas in Iceland.

The course has over the years become known throughout Iceland, and this has contributed to strengthen young women's awareness of the possibilities of starting their own business as an alternative career path - even in peripheral areas, where women's employment opportunities often do not match their educational qualifications. This project has helped to maintain some women in peripheral areas who would otherwise most likely have looked to larger cities to find jobs. The project has 
also been highlighted in connection with an EU-funded project on "Female Entrepreneurship in Nordic Regions", which aims to promote female entrepreneurship in Norway, Iceland and Denmark.

The course in entrepreneurship is offered twice annually, and has a duration of 15 weeks, which entails course attendance for 6 hours one day per week. The course is offered in Reykjavik, Akureyri and in selected peripheral areas.

Read more at:

- http://nmi.is/frettir/2013/12/brautargengi-byr-til-verdmaeti/

- http://www.nordregio.se/en/Metameny/NordregioNews/2013/Gender-Equality-for-Regional-Growth/Reflection/

- http://www.diva-

portal.org/smash/get/diva2:700352/FULLTEXT01.pdf 



\section{Changing Gender Relations and Gender Norms}

In this section we focus on the meaning of gender and how research and literature on the subject illustrate how the places men and women live, and the spheres they frequent (e.g. the family, the educational institutions, the workplace, etc.) all are rooted in specific geographical locations that help define both which structures of opportunities exist, and what degrees of freedom the individual experiences. As it will appear, part of the literature points to how women's desire of moving away from the peripheral areas, to some degree, is combined with the women's wish to gain access to more opportunities as well as a bigger sense of freedom (or less social control).

\subsection{Sweden}

In a Swedish context, Forsberg $(1997 ; 2001)$ has, with inspiration from Hirdman, worked with what she calls local and regional gender contracts to illustrate certain tendencies in the Nordic peripheral areas. Forsberg defines gender contracts as unwritten rules and norms that regulate the relations between men and women, and that contribute to the creation and maintenance of gendered structures of opportunities in these areas. In her work, Forsberg has additionally used the term escalator regions (rulletrapperegioner) to describe the Swedish regions close to the cities, which, in opposition to the peripheral areas, are dominated by what she calls contemporary gender contracts meaning a less traditional gender division and a less traditional perception of the opportunities and freedom of choice for men and women. Forsberg has later, together with Stenbacka, taken the notion of gender contracts even further. In a collaborative publication, they explain the use of the term "gender contracts" as follows:

Gender contracts are shaped by a combination of the overall structure of gender relations and the way in which they have been arranged by local conditions in the labour market, demographic structure, history and traditions.

These local gender contracts are the everyday practices that people perform, 
mostly without noticing or being aware of them. By emphasizing external or structural conditions, we do not ignore agency or the actors' own intentions and goals. Structures are built up and maintained by agency, and individuals can affect structures by negotiating and challenging existing norms. From time to time, people comment on the way in which these gender contracts guide everyday lives in their area. They also express how the gender contracts are locally constructed. On other occasions, the specific contract is less explicit (Stenbacka and Forsberg 2013: 14).

Forsberg's (2001) and Forsberg's and Stenbacka's (2013) point is that when women decide to move away from the peripheral areas, it can be due to, not just, a career strategy but also a gender equality strategy. When young women choose to relocate it is based on a combination of own will, capacity and dreams and also on what is perceived as possible and obvious on the basis of, for instance, local (gender) traditions and gender perceptions. In other words, perceptions of how young men and women, respectively, ought/can/are expected to behave - in other words, on the basis of the local and regional gender contracts.

Also Waara (1996; 2003), who has studied young people in five municipalities at the Swedish-Finnish border, concludes that the youth in the peripheral areas have different strategies for the future, and that these are not just related to their attachment to the local community but also to their concept of gender relations and gender norms. Waara concludes that the gender identity of the youth contributes to their feeling of being in or being out of the local context. Thus, he finds that the young people, who have a traditional gender identity, to a greater extent, are involved in and connected to the local community, as opposed to the young people with a "modern" gender identity who display a weaker place attachment. Waara additionally concludes that young women feel more tied up with the locations and the gendered position they are offered, and that this contributes to their desire to move: "Insofar as young women have more "to gain" than young men by leaving the asymmetric gender roles, an explanatory dimension around the skewed sex ratio is established at the cultural level" (Waara 2003: 198, own translation).

Svensson (2006) has worked with Forsberg's perspective on gender contracts and on the basis of a study of the Swedish city, Söderhamn, concluded that the local and regional gender contracts can take different forms if one also takes a class perspective into account. It is all about the fact that the conceptions and the expectations of the two genders vary because of the interplay between gender and class. According to Svensson, the often very traditional gender contracts (the social ground rules) in the peripheral areas do not cause problems for the young men. How- 
ever, she does find that the local and regional gender contracts divide the group of young women:

\begin{abstract}
It is among the group of girls that the biggest differences are to be found in terms of what they see as important in life. [...] The girls with a working class background tend, in their thoughts about the future, to focus on security and family, and in other matters they are more diffuse and flexible, which can be seen as evidence of a traditional female role. [...] Girls who accept/appreciate the traditional female role perceive a future for themselves in the region [...] The group that most clearly distance themselves from staying in Söderhamn, and they do so emphatically, are the girls with middle-class backgrounds. For them, there is no ambivalence. Most of them believe that, without a doubt, there is no future for them in Söderhamn within any foreseeable time frame (Svensson 2006: 145, own translation).
\end{abstract}

Also Rauhut and Johansson (2012) and Rauhut and Little (2014) have written about gender differences in mobility patterns when looking at the Swedish peripheral areas. They have, in connection to this, concluded that the patriarchal structures constitute a significant push-factor for the young women in the peripheral areas:

Traditional gender structures and "macho" lifestyles are often heard motives that influence the out-migration of young women from rural areas (Rauhut and Johansson 2012: 1).

Young, well-educated women may refuse a traditional way of life and family formation which is associated with rural living and attach great importance to gender equality. Gender issues in rural societies, e.g. the construction of rural femininities and masculinities may be relevant for migration since traditional gender roles and expectations are more prevalent in rural societies (Rahut og Littke 2014: 5).

According to Rauhut and Littke, the so-called macho-culture in the peripheral areas can be a factor which more or less indirectly affects the young women's decision to move. However, in contrast to this, Stenbacka (2011) writes that the idea of the rural macho-culture, which is also underlined in the Swedish TV programmes she has studied, can be seen as an attempt to place the blame for the problems in the peripheral areas on the men, who choose to stay there (elaborated later) (see also Forsberg and Stenbacka 2013). 


\subsection{Norway}

Dahlström (1996) argues, in the often quoted article Young women in a male periphery, that the strong patriarchal structures embedded in the peripheral areas are part of the explanation for the fact that particularly the young women decide to move away. Thus, Dahlström writes that women are not only attracted by the possibilities found in the urban areas, but the women are also particularly "pushed out from a male rural area in which there is little place for them culturally or concerning work opportunities" (Dahlström 1996: 262). According to Dahlström, the peripheral areas are constructed as masculine because of local power relations, (male) dominating values and norms, as well as centred on activities that are performed and dominated by men, while the women's activities both are less visible and are ascribed less value. Dahlström finds that in the Norwegian peripheral areas there is an increasing cultural gap between the "modern" women and the "traditional" women, and that this, in the long run, can lead to a situation where "the young men may be left behind as losers (and) become marginalised" (Dahlström 1996: 270). Dahlström's overall conclusion is - in keeping with later research from a Swedish context (cf. above) - that it is the "machoculture" in the peripheral areas that plays a part in pushing the young women away.

The Norwegian researcher, Grimsrud (2011) speaks of the term regional gender contracts in relation to understanding the interaction between gender relations/norms and mobility. Grimsrud (2011) speaks of three different types of women who are located in the peripheral areas, and argues that these different types of women respond differently in relation to the gender contract in the peripheral areas. Thus, Grimsrud operates with the traditional, the modern and the alternative. Grimsrud emphasizes that particularly the women who belong to the traditional category, move to the peripheral areas to have more time for family life, and a number of women also choose to work part time. The modern woman who moves to the peripheral areas is often attracted by a particular job opening, which is seen as an important step in a better future career. The alternative woman who moves to the peripheral areas, is, according to Grimsrud, not tied down by traditional gender roles, but chooses, to a great extent, the location based on a desire of being closer to nature and to live another kind of life than the city life (Grimsrud 2011).

Both Rye (2007) and Bye (2009) accentuate that gender always has been an important variable in relation to shedding light on why young people move away from the peripheral areas. Therefore, Rye points out 
that young women and men from the peripheral areas have different motives for moving. However, prevailing gender relations and norms play an essential part in understanding their choices - particularly the choices made by the young women:

For example, the relation between gender, rurality and relocation are probably different in various types of rural communities. "Masculinity" gives rise to other associations in the most tradition-infused agricultural municipalities than in coastal villages, or in rural communities that have industrial activities as their economic foundation. The farmer, the fisherman and the rural industrial worker, or what is the most common occupational representatives in today's rural Norway: the public employee and the service sector employee, all carry different and gendered connotations. Gender, rurality and relocation are all context-dependent concepts (Rye 2007: 95, own translation).

Despite the fact that rural men are gradually becoming more involved in the home arena, the rural district is still to a large extent a place for gendered divisions of labour. To a great degree it is still the women who "choose" to work part-time in order to look after their house and home because their "old man" has so much to do. Furthermore, despite the fact that rural women have expanded their feminine identities by adding paid work to their traditional tasks, they still remain in traditional gender roles and relations. In other words, it seems that the "traditional" gender system, to a large extent, is making it difficult for young men and women to break with established gender patterns (Bye 2009: 286).

Another Norwegian researcher, who has studied gender relations and norms in northern Norway, is Wiborg (2003), who on the basis of an interview study with young men and women from smaller towns in northern Norway, conclude that several of the young women perceive the act of moving from the small local communities in the peripheral areas and obtain an education as determining for the possibility of shaping one's own identity and creating a life style free from local and cultural limitations. This includes freeing oneself from gendered expectations of women's role/responsibility, relationships and motherhood - expectations that these young women see as limiting their space for action:

The young female students are afraid of "being tied down at home" in an almost literal sense. At home inside the house, surrounded by duties towards husband and children. The emphasise the idea of moving and obtaining a higher education as a way to gain control over the shape of their lives - in contrast to those who stay behind, become mother's and do not get an education. The idea of motherhood at an early age is perceived as a loss of control over one's time, especially if coupled with a relationship to a locally oriented husband. The idea of staying is associated with a form of disempowerment and lack of control over one's own life without the option of moving on, both 
geographically and socially, and with limited possibilities for "finding oneself" and "being oneself". Moving and the wish not to be tied down locally, is therefore also a choice they associate with a question of lifestyle (Wiborg 2003: 350, own translation).

Gerrard (2013) has undertaken studies on Norwegian fishing communities in Finnmark in northern Norway, where she, among other things, has examined the question of changes in the expectations for the fishermen, for instance, in relation to mobility, but also concerning the breaking up of traditional gender roles on the basis of changed living conditions and restrictions relating to quotas and other regulations of the fishing sector. Gerrard emphasizes that the gender norms are changed by virtue of the fact that the expectations for both men and women"s performance are rising in the fishing towns. Gerrard underlines how the men now - apart from being qualified fishermen, who need to be able to adjust to a global market with regulations and fluctuating structural conditions - are also expected to be present as father's, to take an active part in their children's lives; as well as good life partners taking an active part in family life. Thus, Gerrard concludes that the social distinctions between the fishermen at sea, their abilities as father's and husbands, along with the women's roles ashore as wives, mother's and professionals are changing:

\footnotetext{
Unlike in the past, when mobility practices appeared to be strongly dictated by gendered relations and social reproduction imbedded in a male breadwinning worldview, today's practices demonstrate that instead power is not locatable to one "gendered" source but rather is everywhere in daily life, in speech, and in knowledge. The weakening of these boundaries has consequences also for undermining the boundaries between fishers' physical and intellectual work and between different places, including fishing grounds. Gendered differences in mobility practices still exist, but the criteria for masculinities and femininities seem to be continuously changing (Gerrard 2013: 318).
}

\subsection{Denmark}

In the light of a study of young people in Skagen, Bjerring $(2000 ; 2007)$ draws the conclusion that young women in the periphery seem to be more mobility minded compared to young men which, among other things, is due to the fact that young women are relucatant to walk in their mother's footsteps both in regards to choice of profession and family life - they see these "hinterland women" a female figure belonging to the past. Thus, the girls' increasing orientation towards education must, according to Bjerring, be seen in correlation with an increased orienta- 
tion towards gender equality and a wish for liberation from the traditional gender patterns both within the family and on the labour market.

Bloksgaard (2011) focuses in her research on gender segregation in the labour market on how perceptions of masculinity(-ies) and femininity(-ies) are essential parameters to look into in relation to analyses of the labour market. The stereotypical ideas of men's and women's jobs are, by way of example, expressed in the retraining of men for care work. As pointed out previously (Baagøe Nielsen 2014), there are certain challenges associated with getting men to do this kind of work, as there is often an embedded stereotypical idea of care work being jobs for women.

As stressed by Hansen, in the peripheral areas there is a list of traditional professions, which are often marked by a specific gender - in Hansen's case, it concerns the fishing industry that traditionally has been (and still is) characterized by strong gender segregation. Hansen emphasizes that the traditional local professions are often more aimed at the boys, and that the girls therefore must make a deliberate effort if they want to get into a "male job" as e.g. fishing. Therefore, it is often easier and more natural for the boys in the local communities to take over their father's professions, because the societal norms to a greater degree expect the boys to take over, for instance, their father's fishing boat. This creates a close connection between the boys and the local community and the occupational traditions effective in the area. At the same time, it can complicate the choice of going for another education/profession, because this in most cases will imply an opting-out of the local occupational traditions. Likewise, Hansen underlines that it will apply to a significant number of the boys that they will be the first generation to get an education. And, at the same time, this entails a break with generations and traditions within a certain profession, which is also a consideration that comes into play when the boys choose an education

It is not only in relation to the labour market and the upskilling that gender relations and norms have an impact. In their chapter on boys' experiences and routes through the educational system, Nielsen and Jørgensen focus on problems with stigmatization related to boys and education. They analyse perceptions of the boys' problems in school with a view to understandings of masculinity. In their introduction, Nielsen and Jørgensen stresses that boys today perform worse than the girls in school, and that at the same time there are fewer boys than girls who graduate from secondary school. Furthermore, the women account for the majority of students in higher educations, including in prestigious programmes where men previously have been dominant, such as in medicine and political science (Nielsen and Jørgensen 2013). 
Nielsen and Jørgensen question the manner in which the educational system and politicians have approached the matter of "the problem with boys", and argue that the discussion of boys' problems in school often combine gender politics and social politics in grand narratives that often draw on one-dimensional and stereotypical ideas of boys and men. When such narratives and ideas lay the foundation for education politics, there is a risk of enhancing the schools' gendered problems rather than solving them (Nielsen and Jørgensen 2013:11).

\subsection{Finland}

As specified earlier, Lapland constitutes a distinct part of the Finnish debate on the peripheral areas, population flows and gender. Westman (2005) underlines in her research that Lapland particularly is a periphery, and that this is evident both geographically but also mentally among the Finns (Westman 2005: 114-115). Furthermore, in her research, Westman argues that there is a clear gender dimension to Lapland's geographical placement, and that this plays a part in relation to the national (and local) understanding of Lapland and for the local gender relations and gender norms. Thus, Westman points out that there is a prevailing traditional narrative of Lapland being a place where strong men travel to cope in the wilderness, and where women simply are in transit. Hence, the entire region has a masculine imprint:

The present myths of Lapland are represented as masculine by the media. The real men go to the North where he can survive in the heavy nature. Woman is a visitor in those stories as honored mother or not-valued entertainer (Westman 2005: 114-115).

The masculine culture may be the explanation for why particularly young women move away from the Nordic periphery, and why young men often remain. At the same time, Westman argues that in these locations new gender relations and gender norms are created as care work for parents and children, which the women otherwise traditionally have dealt with, is now also managed - and in some cases completely taken over - by men. Westman points out the fact that the changed gender relations and gender norms in Lapland, and also in Finland, call for more research.

The Finnish researcher Peltomaa (2013) has also brought the gender relations and gender norms in Finland into focus; however, with a specific focus on homosexuality, and the complications that can be tied to being homosexual in Finland, particularly in the peripheral areas. Pel- 
tomaa underlines that traditional gender role patterns to a great extent form an essential part of the Finnish culture, especially in the peripheral areas, which leads to the fact that it can be difficult to live in the more peripheral areas if you deviate, for instance, by not following the traditional gender roles and have another sexual orientation than the majority. Peltomaa refers to how this complex of problems has been presented in the Finnish media over time:

About half a year after (beginning of 2009, ed.) [...] the Finnish Broadcasting
Company, Yleisradio, released a news item: "Lack of gay culture drives people
from rural areas to Helsinki". And then in 2010, the leading newspaper in the
province of Lapland, Lapin Kansa, also wrote how "homosexuals from Lapland
are escaping to the south", pointing out that the term "sexual refugee" is a
common expression for homosexuals from Lapland (Peltomaa 2013: 155-156).

As described by Peltomaa, it can be difficult to be accepted in the peripheral areas if you are homosexual, and therefore some (young people) decide to move to e.g. Helsinki. Particularly Lapland, Peltomaa underlines as an area where it can be difficult to be homosexual because of varying negative associations. At the same time, the peripheral areas are often areas where the community is strong, and where there is a significant attachment to family, friends and neighbours. Therefore it can be difficult not being part of the majority. This means that a number of young people relocate from the peripheral areas as they often feel more accepted in bigger cities. Peltomaa stresses that the skiing areas in e.g. Lapland are an integrated part of life in the Finnish peripheral areas and a massive part of the livelihood. Apart from that, the ski areas are also places where people can escape the dense and close-knit life of the small Finnish villages. This also means that the ski areas have a great influence on the peripheral areas in, for instance, Lapland. Peltomaa writes:

The most notable difference stems from how near or far a village is located from a town or ski resort. Life in a geographically-isolated village is quite different from life in a village that can accommodate 23,500 visitors during the ski season (Peltomaa 2013: 158).

Therefore, it can be of great significance for young homosexuals if they live close to a ski resort in the periphery in relation to feeling accepted in society, which, according to Peltoma, has influence on whether they choose to relocate to bigger cities, or whether they choose to stay in their local communities. 


\subsection{Iceland}

Rafnsdóttir and Ómarsdóttir (2010) sum up their research on women and welfare in Iceland by pointing out that even though the country has been a pioneer country concerning gender equality (for instance, giving women the right to vote, passing a gender equality law, earmarking parental leave for men and introducing gender quotas among chief executives), it cannot be said to be the Promised Land for women. The results of Rafnsdóttir's and Ómarsdóttir's interview study among a number of Icelandic women thus point to an unequal distribution of the responsibility for the family and the home in Iceland, where the women in the study repeatedly stress the lack of real gender equality in Icelandic society. A lack which is also reflected, they argue, in the huge gendered wage gap on the Icelandic labour market. Terms such as "social control" and the fact that the Icelandic population is so relatively small are mentioned several times in their study. At the same time, Rafnsdóttir and Ómarsdóttir call attention to patterns of high birth rates and a high level of education among Icelandic women, which often lead to unfortunate consequences for women's position in society:

They (the Icelandic women) tend to become mother's at a very young age, compared with women in other western countries, and they may have their youngest child when they are in their forties. This pattern has led many Icelandic women to still have young children when they have to start taking care of their own parents, now getting older. They also pointed out that it is not uncommon for Icelandic university students to have children, and many buy their first apartment while they are still studying. This is often perceived as positive and as a testimony to their hard work ethics and drive, but the women themselves generally see it as a sign of lack of planning and sheer greed which can put pressure on families, especially on women (Rafnsdóttir and Ómarsdóttir 2010: 76, own translation).

Iceland is the country in the Nordic region where father's use most of the parental leave with their children - thus, father's use 28.5 per cent of the joint length of the leave (Haagensen 2014: 64). But, Rafnsdóttir and Ómarsdóttir underline, by referring to Einarsdóttir (2004) "the women still take more parental leave than men, and the law has not eradicated the gender pay gap" (Rafnsdóttir and Ómarsdóttir 2010: 82, own translation). Edvardsdóttir writes about Icelandic gender norms that: "Despite the traditional ideas about masculinity and femininity, all women claimed to be equal-rights minded. They said they were not feminists because in their minds that concept had a negative meaning" (Edvardsdóttir 2013: 83). 


\subsection{Greenland}

Eistrup and Kahlig (2005) point out that in relation to the question of gender equality in Greenland, the specific situation concerning the introduction of Greenland's Home Rule must be taken into consideration, whereby the question of gender equality at the beginning was subsumed the process of ethnic equality between Greenlanders and Danes in the political process that led to the introduction of Home Rule in 1979. However, they also point out that after the introduction of Home Rule, there has been an increased focus on the importance of gender equality.

Eistrup and Kahlig (2005) underline that current patterns in gender segregation, in terms of choice of education and affiliation with the labour market in Greenland, to some extent can be seen as offshoots from the colonial time and the changes it brought with regards to the relationship between Danes and Greenlanders - as well as internally within the population of Greenland. They also point out that over the years there has been a certain disassociation with the phrase feminism among Greenlandic women as well as women's organisations (Eistrup and Kahlig 2005: 206). In contrast, they refer to "an approach to the equal value of both genders based on consensus" (Eistrup and Kahlig 2005: 208, own translation).

To understand the underlying mechanisms that contribute to explaining the strong gender segregation on the Greenlandic labour market, along with choice of education and patterns of mobility, it is rewarding to take a closer look at the prevailing norms regarding the relations between the genders. As depicted above, Poppel, among others, points to the modernisation period of the $50 \mathrm{~s}$ and $60 \mathrm{~s}$ as stumbling blocks for significant changes in the gender relations in Greenland. About this Weyhe writes, "there is reason to assume that problems associated with integrating Greenlandic men in the modern labour market are related to predominant cultural ideals and past and present power relations in Greenland. However, this does not seem likely to be the only explanation" (Weyhe 2011: 255, own translation). However, there is also reason to assume that changes are under way. The report from Nordregio thus accentuates that there seems to be increasing status associated with paid work outside of the traditional professions (Nordregio 2009). 
Just like Greenlandic men obviously differ among each other, the same naturally goes for Greenlandic women. With respect to the women, the available norms concerning how a young woman can or ought to act, however, seem more broad-spectred - at least as concerns choice of education and establishing a family. On one hand, there is thus a tendency for early motherhood, and traditional gender role patterns that are perhaps especially present in the small villages. On the other hand, this is in some cases simultaneously connected to pursuit of further education. According to Eistrup and Kahlig:

Within the traditional hunting and sheep rearing district we still find a number of stay-at-home mothers, who partly help their husbands with their work and partly take care of home and family. As many women have their first child at a young age, it is still a common practice for the grandparents to help with raising the kids if their daugthter decides to pursue education in another town (Eistrup and Kahlig 2005: 211, own translation).

Poppel (2010) in her interview study among young women located in Nuuk, asked detailed questions of how they would explain women's choice of moving from the small villages to the towns and potentially continue to Denmark. She reports on the basis of these interviews that the young women who did not have children expressed that "a reason for moving away may also be that the population is too small, and that therefore women do not feel sufficiently inspired in their everyday lives" (Poppel 2010: 48, own translation). As concerns explanations for further moving entirely out of the country, the interviewed women for instance pointed to housing shortage in the towns, as well as inadequate public services, for instance for children with disabilities or special needs.

In overall terms, there is no doubt that the different norms and expectations for the two genders indicate a general picture of segregation in the fields of work and education in Greenland. And a number of different reports concerning, for instance, the well-being of young people (Pedersen and Bjerregaard 2011) and women's welfare (Poppel 2010) point to a great need to increase support for boys and men. This is accentuated because the survey on the well-being of young people shows that boys have difficulties talking for instance to their parents about potential problems, and partly because the suicide rates are higher among young men. 


\subsection{The Faroe Islands}

According to Knudsen (2009), gender equality has been a topic of discussion for a long time in the Faroe Islands, and particularly women's lack of representation in politics and in business life - however, without it having led to significant changes. About the beginning of the work for gender equality in the Faroe Islands, she writes that, "it took many years from the decision in 1976 to establish a gender equality committee and until the policy proposal to have gender equality was presented in Lagtinget (Parliament, eds.). The Faroese law on gender equality was approved in 1994, and with it, the first gender equality ombud in the Faroese was established" (Knudsen 2009: 322, own translation). At present, a law on gender quotas in public councils, boards, committees and commissions has been introduced through the Gender Discrimination Act, yet this is also, "one of the most controversial and discussed themes in the debate on gender equality in the Faroe Islands" (Knudsen 2009: 323, own translation).

The limited role of the Faroese women in politics is also discussed by Jacobsen and Jákupsstova (2005), who argue that "women's role in the Faroe Islands is changing". They emphasise that Faroese society, as a whole, traditionally has been gender segregated - admittedly, not in a legal or a religious sense, but rather in a practical sense "the women have main responsibility for unpaid labour within the family, and the men dominate public space" (Jacobsen and Jákupsstova 2005: 170, own translation). Like Knudsen, Jacobsen and Jákupsstova also write about the public gender equality debate, and conclude in this relation that "somehow the debates seem to miss the point and are more or less getting nowhere" (Jacobsen and Jákupsstova 2005: 194, own translation).

In 2006 a committee by the name of "Demokratia" was set up in the Faroe Islands, whose purpose, among other things, is to motivate women to take part in politics. Of this Knudsen writes that "the role and work of the committee has led to debate within the Faroese society and is (perhaps) one of the few visible changes which Faroese political authorities have started to support the advance of gender equality in society" (Knudsen 2009: 324, own translation). Knudsen's interview-based study of women's well-being in the Faroe Island and their attitudes to welfare society, however, also shows that the Faroese women "do not recognise themselves in the work undertaken by the equality committee, nor in the way questions of gender equality are being treated in the public debate (in the media)" (Knudsen 2010: 23, own translation). But even though 
the Faroese women are not pleased with the content of the gender equality debate, Knudsen concludes in her study that:

\begin{abstract}
Gender equality and equal opportunities are important for livelihoods and well-being, but in terms of whether they choose to settle in the Faroe Islands or abroad, the economic factor is the key. The economic factor in this context means, mainly, the support or the welfare services that they are entitled to in Denmark compared to the welfare services they can get in the Faroe Islands (Knudsen 2010: 24, own translation).
\end{abstract}

Closely connected with these economic considerations, in relation to the decision of relocating or not, is the question of education that the Faroese citizens have direct and free access to in Denmark on the same terms as the Danish population. ${ }^{13}$

\title{
5.8 The Åland Islands
}

It has not been possible to find research literature directly concerned with potential changes in gender relations and gender norms in the Åland Islands relating to education and population flows. From a gender perspective, however, it is interesting that Ålands Statistik och Utredningsburå (ÅSUB) since 2012 has compiled an annual report concerning essential gender differences in the society of the Åland Islands. The politicians of the Åland Islands requested that the reports have different focus varying from year to year, for instance, on the labour market and differences in the economic situation among women and men in the Åland Islands (2012), differences in men's and women's conditions in society and work life, including economic differences (2013), as well as gender differences in relation to heath, illness and care (2014). The focus of the report from 2015 was on children and the youth, and the gender divisions that occur already in early childhood and youth, e.g. in family conditions, choice of elective subjects in school and experiences of well-being during school age.

The gender segregated data from ÅSUB has documented gender inequality on a number of parameters (NIKK 2014), and in the rest of this

${ }^{13}$ Like all other Nordic citizens, http://www.norden.org/da/norden-for-dig/uddannelse-inorden/uddannelse-i-danmark/optagelse-paa-hoejere-videregaaende-uddannelse-i-danmark, date of access: March 9th 2015. 
section of our report, some of the social parameters for gender inequality of the Åland Islands are highlighted.

Women take almost all of the parental leave in the Åland Islands also when looking separately at the part of the leave that can be shared between the parents. "In practice, this means that it is also the women who take main responsibility for the home within the Ålandic families with children" (ÅSUB 2015: 7, own translation). A report from ÅSUB points out that this most likely, among other things, is due to the fact that the women generally earn less than the men, which can "influence the decision on how to share the parental leave and responsibility for caring for the children within the family, as the parental leave benefits are calculated on the basis of income" (ÅSUB 2012: 4, own translation). Thus, the women have the primary responsibility for care work in society - both within the families and as employees in the public nursing and care sector

A report from ÅSUB calls attention to the fact that there are "similar but rather larger gender differences in Åland compared to the rest of the Nordic countries" (ÅSUB 2012: 8, own translation), and that there, at the same time, is a tendency that the role models children in the Åland Islands meet during their school life also follow established gender norms in relation to whom, for instance, teaches language subjects (86 per cent female teachers) and woodwork (100 percent male teacher) (ÅSUB 2012: 10). ÅSUB concludes that no major revolt against established gender norms and patterns seems underway.

Best Practice Case: North Atlantic Law Programme (NALP) - Denmark, Faroe Islands and Greenland

"North Atlantic Law Programme" (NALP) is a collaborative project between Aalborg University in Denmark, Greenland and the Faroe Islands. The program makes it possible for students in the Faroe Islands and Greenland to stay at home part of the time while studying law at Aalborg University (inter alia through transmission of lectures). The goal has so far been to establish the first semester of the Bachelor of Law degree in Greenland and the Faroe Islands in the autumn of 2014. While the program ensures that young people stay in their local area during substantial parts of their education, the education also focuses on offering electives related to the judicial systems in the North Atlantic, especially Faroese law and Greenlandic law. This makes it possible for students attending the graduate programme to 
choose subjects that are immediately qualifying for positions where knowledge of judicial aspects of the Greenlandic and Faroese society is required. Graduate students have the opportunity to write their thesis focusing on legal principles from the two areas. In addition, there are ongoing efforts to develop and support the research environment in Greenland and the Faroe Islands, including by incorporating PhD students in the programme.

The process of spreading information on the NALP began already in 2011, when leaflets were distributed at four high schools through the "sea show" (a boat sailing along the coast of Greenland). The project includes consideration of peripheral issues in relation to the training and retention of young people in remote areas, where especially young women choose to take a higher education and thus also move. One effect of the project can thus be that young Greenlandic women (and men) are more likely to remain in the immediate area and in higher education through online courses. So far, there has been a predominance of women enrolled in the programme.

The number of students in NALP currently (spring 2015) amount to:

- 2nd semester: 3 students, (2 female, 1 male)

- 4 th semester: 4 students, (girls) (the first real intake of students in the programme)

- 6th semester: 2 students (girls)

Both the bachelor and master programme include an offer for the students to attend examinations physically at Ilisimatusarfik (the Greenlandic University). This enables students in the program to be physically located in Nuuk (or where applicable, other parts of Greenland) during the winter exam period (December and January) and summer exam period (May, June, July and August). The expenses for student travel is not funded by the program, but can be fully or partially funded by the Greenlandic Self-Government annual return system in cooperation with the Greenlandic House in Aalborg. NALP is a law programme, but the model also holds obvious potential to spread to other areas of study, and other Nordic countries.

Read more at:

- http://www.law.aau.dk/den-juridiske-skole/north-atlantic-lawprogramme/ 


\section{Contact persons:}

Leader of The North Atlantic Programme, Associate Professor Louise Faber - faber@law.aau.dk or head of studies Hanne Søgaard Ilisimartusarfik hsoe@uni.gl

Best Practice Case: Project Men in Health - Norway

The project "Menn i Helse" (Men in Health) is a Norwegian example of a best practice case that addresses challenges related to the retraining of men from typical "male jobs" to perform jobs in the care sector. The project was initiated by Trondheim Municipality in 2007, and had from its outset a strong focus on solid evidence about men in care work, before the project was finally launched. The mapping of previous experiences was launched during 2008/2009, and in 2010 a project manager was appointed. Thorough preparation, including analysis of the target group and the involvement of employees at various levels (local, regional and state), is one of the reasons why the project has been a great success. The project has succeeded in creating awareness of social care as a career path for men, as well as increased recruitment and retention of men in the care sector to an extent never experienced before in Norway. The great success has led to the fact that the project has spread to several other municipalities.

The project has used various approaches. For example, it has offered 8 week long internships, training and not least extensive campaigns that have been able to capture and maintain interest among the target audience. A key part of the work related to communicating about the project was the formulation of a new concept for people working in the care sector. Hence, the project took on the name "helserekrutt" (health recruit), deliberately intending to appeal to men. To increase awareness of the project, a number of unemployed men were initially recruited to work as "health recruits" in internship positions. These men were then intended to help recruit more unemployed men otherwise receiving unemployment benefit.

In addition to its efforts to create campaigns and educational materials, the project has developed a training model that targets the group's needs. The model was tested during the period 2013-2015 on 20 selected men. Participants have been selected jointly by NAV and Trondheim Municipality. The training will result in a diploma as health worker, however, the program needs to be approved by the Educational Directorate first (currently ongoing). The education model and the project as 
a whole have managed to involve both local, regional and national parties. The project does not contain a specific focus on peripheral regions, but can be used as inspiration for meeting some of the challenges that peripheral areas are facing in relation to changing living conditions and job opportunities. The project's success in training unemployed men for jobs in the health sector is interesting, because many peripheral areas in the Nordic countries have a high demand for staff in the health sector, while many men in remote areas find that the traditional male occupations are disappearing fast.

Read more at:

- http://mennihelse.no/ 


\section{Young Men and Masculinity/- ies in Peripheral Areas}

As described in the beginning of this report, the literature shows that the men in the Nordic peripheral areas seem to be particularly affected by the restructuring of the labour market in the peripheral areas, and the changed living conditions, which are related to these changes. It has also previously been highlighted that the financial crisis of recent years has advanced this development more rapidly, which is why one does not talk about the recession, but about the mancession from an equality perspective. All of this should be considered in combination with the fact that today there is a strong focus on mobility and flexibility, which, according to the research literature, contributes to creating a special narration about the men "staying behind" in the peripheral areas. In that debate, this group of men - and this seems to be the case across the Nordic countries - has been described as hostile towards transitions, uneducated and as losers, who are out of touch with the development/evolution of society. The fact that young men residing in the periphery may happen to have made conscious and deliberate decisions about their residence, education and profession, is to a lesser extent included as an element in the cross-Nordic debate.

\subsection{Sweden}

Stenbacka (2011) has explicitly considered the images of men in the peripheral areas as presented in the Swedish media and in Swedish TVshows and films. She concludes that this group of men often is referred to ruthlessly. She finds that the peripheral areas often are presented as help-seeking and backward, that the men in the peripheral areas are depicted as unequal and traditional, as marginalised, and at times even as deviant. In another publication, Forsberg and Stenbacka also write that:

\footnotetext{
In Sweden, the rural man has become a recurrent actor in diverse media, reproducing male stereotypes. The media image of the rural man is a stereotypical expression of an almost alien, exotic figure [...] Rural masculinity is presented as raw and violent, but at the same time rather childish and antiintellectual (Forsberg and Stenbacka 2013: 2; 3).
} 
In continuation of this, Stenbacka argues that the representation of the peripheral areas can be seen as an attempt to place the blame for the problems of the areas on the men, who choose to remain there. However, it is dangerous to regard these images uncritically. The problem is, so Stenbacka states, that the images risk having consequences, especially because it is highly likely that they will influence the politics which is pursued in the area:

The relationship between the rural and the urban is always present in local as well as national politics, and the way we learn about spatial gender relations and the extent to which "othering processes" prevail will affect people's experiences in different areas, as well as suggested policies [...] If some areas and groups of people are perceived as unproductive, this may have an impact on national politics, on expectations and financial support, and on different kinds of financial support. Support for something strong, viable and positive is different from subsidies and support for what is perceived as stagnant and backward (Stenbacka 2011: 242; 243).

Stenbacka argues that it is generally a problem that the focus which is directed towards men in the Swedish peripheral areas, implicates an attention to the areas' local culture and the men's choice to stay as something which needs to be changed (modernised), rather than something, which should be nourished and preserved.

\subsection{Norway}

The Norwegian researcher Bye $(2009 ; 2010)$ has studied young men in the peripheral areas of Norway with a special focus on their masculinities and sense of identity:

Several studies in recent years have highlighted how young rural women have coped with modern ways of life better than young men, due to the fact that they have sought higher education and increased social participation [...]. While working class male youths for the most part have followed in their parents' footsteps and sought employment within traditional masculine occupations in shipyards and forestry or at sea, female youths have found their way into the "new" labour market in the public sector through its requirements for formal education. When faced with a tougher local work situation characterized by economic failure, structural rationalizations, closures, and also increased demands for formal competence, many young men have experienced the loss of their work-related identity and their pride [...]. Further, many young men have been marginalized in the housing market as a consequence of having low and unreliable incomes. For many young men it has 
been economically difficult to establish an independent life away from home

(Bye 2009: 279).

Bye emphasises that among the young men in the Norwegian peripheral areas, one can trace masculine values, which are highly rated among the men. It is thus significant for young men residing in the peripheral areas, that they are handy/skilled, and that they participate in outdoor activities. Particularly, hunting is seen as an important activity for men in the peripheral areas:

\begin{abstract}
In particular, the extract reveals that hunting and handyman skills facilitate young men's entry into the broader rural community, thus allowing them to secure their masculine identities as "rural men", as they are invited into male networks and other male-dominated activities in the community. Furthermore, it reveals that it is important for young rural men to communicate a particular stance in the ongoing and controversial Norwegian debate on snowmobiles and carnivores, as these topics are related to rural men's loyalty to place. In the following I will explore more fully the importance of hunting and handyman skills in the construction of rural masculinity, as well as the ways in which young rural men who live in a remote rural community "do" identity politics with regard to snowmobiling and carnivores (Bye 2009: 282).
\end{abstract}

As particularly the hunting culture is important in the peripheral areas of Norway, this is seen as a central part of being a man in areas, Bye accentuates that it thus can be hard for men, who do not participate in this type of activity, to be part of the community and the masculine culture surrounding the hunt:

The fact that men may feel "out of place" regarding the rural masculine hunting culture has also been recorded in a separate Norwegian study by Berg (2002). She found that urban men who had moved to rural areas feared that they would not be accepted as "real men" because they did not hunt. In comparing the two case studies it is possible to illustrate some of the fundamental relations between rurality and masculinity referring to "the masculine in the rural" and "the rural in the masculine" respectively (Bye 2009: 282).

Bye points out that it is still possible to be part of the local masculine community if you do not go hunting, but in that case, it is expected that you participate in other types of activities, where some of the same aspects apply; being capable of moving about in nature; being able to endure walking long distances, carrying heavy equipment, etc. It is therefore important that a man who does not hunt can pass the physical and psychological test of hunting. And it is even more important that he is a part of the whole cult of hunting - i.e. that he also takes part in having a 
drink and partying. According to Bye, it is particularly important for the young men to be part of the rural masculine culture:

\begin{abstract}
A non-hunter who makes a fuss about hunting and who cannot face the killing of an animal very much expresses rural masculinity "out of place" - and a man who neither hunts nor likes to "party" is not regarded as a "real" man. Thus, by meeting the physical and emotional "tests" of the hunt, and by enjoying a drink after a long day outdoors, young men display "body qualities" that are seen as central to the notions of what it means to be a "real" man (c.f. Woodward, 1998; Campbell, 2000). In this context, being "out of place" is to be feminized, meaning emotional, sensitive and thinskinned. The denigration of normative traits of femininity is thus rural masculinity "in place" (Bye 2009: 282).
\end{abstract}

Bye concludes that it is not only important to separate the masculine culture from the feminine, but also from the urban masculine culture. The men in the peripheral areas are for instance very aware of how they dress when they go hunting, and they only use natural materials. There is a consensus that real rural men do not use fancy clothes when hunting, but are true to nature and the natural materials, and are casual. In this way, a clear distinction is made between the men, who belong to the local masculine culture, and the men, who are part of the urban culture. It is thus not only important to assume an identity and role as hunter, but it also has to be done in a special way, before one is accepted as a real rural man. Bye argues that this distancing contributes in creating an "us" and "them" culture between the "real" hunters and the "wannabe" hunters from the cities.

Another issue which Bye highlights with regards to rural masculinity is snowmobiles and predators. Snowmobiles are seen as an important part of the rural masculine culture, and they become a part of a narrative about men's skills in applying, working with, and repairing big and powerful machines. Snowmobiles contribute to create a strong masculine identity for the men, as well as demonstrating a willingness for risktaking, which is considered as part of being a man in the peripheral areas: "Thus, the cultural implication of young rural masculinity is to be in control of such machines: a man ought to know how to ride them, how to fix them, and also he ought to be willing to break the laws on snowmobiling" (Bye 2009: 284).

At the same time, Bye points out that the use of snowmobiles and their link to masculinity is ambivalent. It is particularly men in the peripheral areas who think positively about the snowmobiles whereas some of the men in the more populated areas are more sceptical about the use of snowmobiles, and to a certain extent perceive them as being 
part of a "redneck" culture for men in the peripheral areas. The use of snowmobiles and hunting is, according to Bye, a big part of the rural masculine culture, and also a way to be loyal to the local community. The predominant masculine culture, which is based on hunting, outdoor activities, craftsmanship skills, and snowmobiles, can impede that men who for instance have completed an academic education are accepted in the local community.

According to Bye, the most vital issue for the young men is that they can stay in their local areas. It therefore becomes less important which type of job you have, also even if it for example is an employment area which traditionally has had an overrepresentation of women. Bye highlights that the men's positive attitude towards men in traditional "female professions" should not necessarily be seen as an expression of the young men having changed their view on what are male and female professions, but rather, that this is a consequence of the fact that more of the traditional professions in the peripheral areas are disappearing, which is why the young men in the peripheral areas find employment within the service sector, in order to be able to stay in their local areas.

Bye accentuates that the young men who reside in the peripheral areas to a larger extent are flexible with regards to their masculinity than their father's, and that their identity is centered on their jobs in the same way as that of their father's generation. Yet, it continues to be important for young men in the peripheral areas to be interested in fishing and hunting, and in this way, their masculine identity becomes more dependant on their spare time interests than their job: "A young man who does not "master' both nature and technology, will probably have problems with being accepted as a "real" man within rural society" (Bye 2009: 287).

In the book chapter Place Attachment, Unemployment and Masculinity: Young Men in the High North, Paulgaard (2015) concludes that young men who grow up in the same place as their father's, and who mirror themselves in their lives, are confronted with the fact that "the world of opportunities has undergone dramatic transformations" (Paulgaard 2015: 211). In the peripheral areas there are for instance far fewer employment opportunities within the traditional trades than previously, and this not only characterises the places, but also the self-awareness and self-image among the young men, who live here. Paulgaard states:

Changes in the world of work have contributed to a shift from manual work to dependency on social benefits for many young men growing up in rural societies. Although many of these changes are caused by overall economic and structural changes, unemployment is defined as an individual flaw and a failure to take care of one's responsibilities. Despite structural changes on the 
labor market, the rules of conduct and traditional ideals of work have not undergone the same transformations [...] Even though the structural conditions for work are disappearing; the local ideologies of masculinity have not been weakened (Paulgaard 2015: 215).

Rural masculinity has been connected to work in occupations that are in proximity to nature, such as fishing, forestry and mining - jobs not requiring formal education. (This) change (have been) accompanied by a growing emphasis on urban concentration as the only possible future path, and where hegemonic masculinity does not involve "the rural", but rather increasingly views "the rural" as something deviant (Paulgaard 2015: 215).

The young men in Northern Norway, who Paulgaard has studied, are all aware that they cannot expect to have a permanent job for the rest of their lives, if they even succeed in finding employment in the first place, and because of this, many of the young men experience ambivalence. They are very much aware that there were more opportunities in the old days - as one of Paulgaard's informants expresses it: "I wish I had grown up when my parents were young" - indicating that they arguably show signs of what is referred to as "melancholic masculinity" in the research literature. The ambivalence which these young men feel has to be seen in connection with the fact that they have grown up with an understanding of masculinity and hard physical labour as being closely related: "Traditional masculine imperatives regarding earning an income through honest, hard work have been tightly connected to (rural) men's respect and reputation, as well as one's self-respect" (Paulgaard 2015: 215).

\subsection{Denmark}

Hermansen (2011) has written about challenges in a peripheral area in Southern Denmark, more specifically Horne Land on the island of Funen (Fyn). Based on his study of young men and their attachment to the local area, Hermansen finds that the community feeling in the peripheral areas strongly appeals to the young men who live here - also to a larger degree than to the young women. In extension of this, Hermansen concludes that as part of the masculinity constructions of young Danish peripheral men, there is often an idea that "rural life is better suited for men" - and that notion simultaneously assists in making the girls' emigration from the periphery (because of lacking opportunities for girls) understandable. Representations of rural life as being something which to a particularly high degree matches certain masculine values can thereby contribute to explain not only gender differences in young peo- 
ple's experiences of the opportunities in their local areas, but maybe also the fact that girls have a higher tendency to move away than boys. According to Hermansen, the local cohesion is, among other things, sustained through local associations which create a community identity that seems to have the greatest appeal to the young men in the area:

The search for safety is a central element for those persons, who choose to stay [...]. Safety becomes associated with the place, and the community's geographical starting point becomes inseparable from the purpose of the community. The significance of the safety and the emphasis on the importance of the place is apparently also related to the gender of the young people. It is to a higher degree the young men, for whom the safety is essential (Hermansen 2011: 66, own translation).

According to Hermansen, the young men in the peripheral areas explain that they prefer life in the country side because they experience that there are some values which they (only/better) can practice here, for instance the opportunities to buy a house and own a car from a young age, whilst being able to stay in touch with the local networks.

Ebbensgaard and Beck also explain that the fact that boys more frequently than girls choose to remain in the periphery, in no way should be taken to mean that they are defeatist or indifferent: "They simply just like the way of life, which they grew up with" (Ebbensgaard and Beck 2009: 104, own translation). However, this is rarely the image, which is presented of this group of men in Danish media, for instance. At a time where the peripheral areas generally are characterised by emigration, the men who stay are often portrayed as being less future-oriented and less willing to adapt, than those who move away (Faber et al. 2014).

According to the report Room for Men (Rum for mænd) (Larsen, Thingstrup and Andersen 2014), which focuses on young men in vocational training, some of the young men in the peripheral areas clearly recount how they experience that the peripheral areas are drained of meaning and of opportunities - and this affects the young men's identity and their view on the future. In line with Hermansen (see above), Larsen, Thingstrup and Andersen find that cars play a key role for the young men in the peripheral areas:

There are several important factors about owning a car. The car is a classical marker of masculinity [...] The car becomes an asset in the social relation to peers, because you can offer to share the opportunities which the car gives, with your friends - "I'll drive, then". The car becomes an opportunity to make yourself independent of your parents' goodwill and control with regards to picking up and dropping off, and thus to a more independent life. At the same time (the young men in the peripheral areas) also describe the car as a para- 


\begin{abstract}
dox in relation to their place in a generational order. On the one hand, the car makes them more independent, and allows them to drive to educational institutions, to friends, "cruising to check out the ladies" and so on. But at the same time, the car makes them economically dependent on remaining at home with their parents for longer, because there are high costs associated with owning a car. A (gendered) strategy, which these young men choose, is simply to make the car his place and "drive around". As such, the car becomes a symbol of the complex situation, which these young men are in (Larsen, Thingstrup and Andersen 2014: 63, own translation).
\end{abstract}

That the young men in vocational training appreciate the freedom associated with owning their own car, should also be seen in relation to the fact that many of them have long journeys to and from their place of education. Larsen, Thingstrup and Andersen thus explain that the vocational education sites in Denmark are much more dispersed than the secondary education sites. Young people who choose vocational training and a special main course of study, therefore often have a longer journey to the educational establishment, and fewer options than young people, who choose a secondary education:

\footnotetext{
Given that the choices of youth education are gendered, where proportionately more girls choose secondary education and more boys choose vocational training, then it is also differentiated according to gender with regards to the distance which young people must commute daily to get to the educational establishments. And the need for - and the age of - moving (away from home) to attend particular educational establishments and the opportunities to choose gender-untraditionally (also differ according to gender) (Larsen, Thingstrup and Andersen 2014: 54; own translation).
}

\title{
6.4 Finland
}

As has previously been explained, the Finnish researcher Westman (2005) has concluded that the masculine culture in some of Finland's peripheral areas can be an explanation as to why especially young women move away from these areas. Accordingly, Westman describes how new gender relations and norms are created in these places, partly because the men in the area now must take on the care work for instance in relation to the older generations; something which the women traditionally have taken care of. Westman argues that based on this development, a concept has arisen which refers exactly to this group of men - namely Old boys in the North which covers Finnish men between 30 and 50, who "stick" to the peripheral areas, even though they have reached an age, where they ought to/could establish a family. However, instead of establishing a family, this 
group of men remains in the peripheral areas, where they take care of their parents, mainly their mothers. This is partly possible because a share of the men are unmarried and unemployed. Others among the men get married, but not with young women from the area, but for instance with Russian women instead, whom the men meet for example via the internet. Even though the men, by taking on the care work with regards to the older generations, in some sense are carrying out work for the state and hence are contributing positively; challenges also arise in relation to the new labour division in the families/in the homes with for instance abuse of alcohol and gender-specific violence:

\begin{abstract}
In that way "the old boys" save a lot of public money and the responsibility of care is transforming from women to men. Sometimes these boys find brides from Russia, for example, and the happiness of everyday life blossoms. However, rather often, according to interviewees, those adult men living in their childhood home abuse alcohol and are violent towards their mothers. The relationship between boys and mother's is very sensitive, caring and loving with or/and without troubles. When a boy has work, life is easier. In this context the negative sides of gender-contract; living opportunities to have daily bread, is shifting from women to men (Westman 2005: 133).
\end{abstract}

As explained earlier, Westman points out that the changed gender relations and norms in Lapland - and in Finland overall - calls for more research; there is especially a lack of research on men and masculinity.

\title{
6.5 Iceland
}

In a progress report on the development of gender equality in Iceland from 2012, in a separate section on men's equality, the Centre for Gender Equality Iceland states, that "The most significant policy change the Icelandic government has implemented in order to change social attitudes and roles, was the parental leave act of 2000" (2012: 31), particularly in light of the fact that $90 \%$ of all new father's in Iceland made use of the earmarked leave after the year 2000. The report further states:

The (Icelandic) law on maternity/paternity leave in 2000 on the whole seems to have had a positive effect, at least so far as to involve more Icelandic father's than ever before in the caring and raising of their young children (2012: 31).

There is also a new law from 1992 regarding shared custody in case of divorce, which has led to Icelandic parents choosing to uphold the shared custody in $75 \%$ of all divorce cases. Yet, single father's remain a 
rare phenomenon in Iceland (2012: 31). Even though Icelandic father's are more involved than ever before, the report also emphasises that the fact of having children affects the Icelandic father's and mother's differently, and that in the case of the men, a more traditional breadwinnermentality seems to be activated:

Having children has an opposite effect on the working patterns of father's and mothers. The more children a man has the longer his working hours are, while the reverse is true for women [...] many men see themselves primarily as breadwinners, reflected in their long working hours and their degree of participation in childcare and domestic labour, which is less than their spouses (Centre for Gender Equality Iceland 2012: 31-32).

We have found very sparse references to masculinity ideals undergoing change in the Icelandic context in connection with this mapping. Júlíusdóttir et al. (2013) points out that in the aftermath of the financial crisis in Iceland, there has been a series of stories in the Icelandic media about men who leave Iceland to provide for their families, and here they thereby present an example of a relatively traditional masculinity ideal. They explain as follows:

The discourse on Icelanders in Norway portrays men as having been forced to leave their wife and children in Iceland for a lonely life working abroad (... these men) have been constructed as breadwinners, or, more accurately in the crisis context, as "debtwinners" finding work and higher wages in Norway than in Iceland, enabling them to pay household debts (Júlíusdóttir et al. 2013: 272).

The development from the 1990s and onwards, where many foreign women immigrated to Iceland, and often found work in the fishing industry (Skaptadóttir and Wojtynska 2008: 119; Júlíusdóttir et al. 2013), has to a certain extent meant that the deficit caused by the fact of Icelandic women from the peripheral areas moving into the cities, has been compensated by women with a foreign background, who have settled close to the fish factories, to which their work permits have been issued. In several cases, these women have married Icelandic men, and have also often been granted family reunification- in some cases with children who have been left behind in the country of origin, but also with other members of the family. Yet, after Iceland's entrance in the Schengen cooperation in 2000, these options have become strongly limited (Skaptadóttir 2015). Conversely, according to Skaptadóttir and Wojtynska (2008: 119), it is rare that immigrant men marry Icelandic women. 


\subsection{Greenland}

In a discussion of Greenlandic masculinity ideals, Weyhe (2011) states that "in the traditional masculine ideal (in Greenland) there is a clear dichotomy between the free, independent man (traditionally the hunter) and the subordinate wage earner (kiffakken). When put in relation to a modern labour market according to the Western model this fact can act as a barrier" (Weyhe 2011: 253, own translation). Completely new research by Merrild Hansen and Tejsner (2015, forthcoming) also points to the fact that a conflict exists with regards to the traditional profession of hunting and a just as traditional male role, which contributes in keeping the young men from seeking other occupations and education. In their interviewbased study among citizens of Upernavik, the young men express that they possibly could be interested in jobs in the off shore oil industry, but only if they still can supplement their activities with hunting, gatherring and fishing. Thus, Merrild Hansen and Tejsner explain that even though there is a tendency among the young people to look for a more modern life style, including a notion about more equality between the genders, there continues to be a wish for maintaining a traditional hunting and gathering culture - and this is closely related to an identity ideal, which builds on a perception of something masculine and authentically Greenlandic.

Furthermore, Weyhe points out that the process of modernisation in the 1950s and 1960s gave rise to significant changes in the gender relations in Greenland. With a reference to the current situation, he writes accordingly:

\footnotetext{
... the opposition against "the new" can partly be caused by the fact that a big group of Greenlandic men have perceived themselves as bystanders to the development in Greenland. During the modernisation and industrialisation, a big group of Greenlandic men were sidetracked with reference to lacking discipline and qualifications. Instead, many skilled and unskilled Danish construction workers were summoned, just as many Danish civil servants were called in to work in the growing public administration. Danish civil servants were endowed with particularly favourable pay- and employment conditions in accordance with the birthplace criterion of 1964 (abolished in 1990). Thus, the preconditions for ethnic divisions and class divides were established, just as the meeting between Danish men (particularly craftsmen) and Greenlandic women has created conflicts - and incidentally a new crosscultural group in society, which plays a vital role in the development of society today (Weyhe 2011: 254, own translation).
}

Yet, according to Weyhe, there is an emerging view that the masculinity ideal/s in Greenland are undergoing change, and that a shift is on the way among the younger generations in the direction of seeing the man's 
role in the family also as that of caregiver, rather than just breadwinner. As an example of this, Weyhe refers to the fact that:

\begin{abstract}
...the common image of the quiet and contrarian Greenlandic man (... is) increasingly met by alternative images. The household distributed magazine Sila has among other things contributed to focus on more modern, male role models, which challenge the often monochrome portrayal of men as either winners or losers. The alternative images of Greenlandic men depict men who, among other things, seek advice and support on their own initiative, in order to create a better life for themselves and the family, just as men in care roles in the home and in society are highlighted. The alternative images confirm the obvious: that Greenlandic men differ among each other, and that there also are many well-functioning and caring Greenlandic men (Weyhe 2011: 255; own translation).
\end{abstract}

\title{
6.7 The Faroe Islands
}

Like Knudsen (2010) (cf. above), Gaini also highlights the Faroe Islands as a family-based society, but he simultaneously stresses that this is undergoing big social and cultural changes. Thus, he writes that young men's traditional ideals of masculinity are losing value to a large extent, and are replaced by new competing masculinity ideals in today's Faroese society:

For centuries, the man embodied - honoured in popular legends and ballads - Faroese bravery and strength, which aroused admiration amongst the young (...and) Generally, sons experienced greater freedom and higher societal prestige than daughters (Gaini 2011b: 1, own translation; see also Gaini 2011a).

Yet, according to Gaini, this status is changing today in line with the modernisation of society and the impact of globalisation, and he emphasises that it is important to understand exactly how the identities of Faroese men are changing, in order to completely grasp the changes which the Faroese society as a whole is undergoing currently (Gaini 2011a: 164). He thus states:

The boys from the small villages, who often have a background in Christian conservative families of fishermen and workers, relatively early enter adulthood with own wage and independence. Among the trendy youth of Torshavn they are often characterised pejoratively as primitive and provincial boys with a style and norms which are outdated (Giani 2011b: 2, own translation). 
The boys and the young men in the peripheral areas, who enjoyed a high status earlier, have, according to Gaini, lost standing in the Faroese society of today, as it is now expected that the young people are educated and acquire other types of knowledge and skills:

New demands for formal higher education change the rules of the game. Boys from the periphery can hardly sustain social recognition without seriously investing in education capital. Their previous masculinity and pride is, today, ironically, the perhaps biggest obstacle for a general hero status amongst their peers. Without academic knowledge [...] many boys from the settlements risk ending up as stigmatised societal losers (Giani 2011b: 2).

In the article Once Were Men, Gaini (2006) develops four typologies of young Faroese men. He argues that Atlantic cowboys and Urban youth are the most widespread masculine ideals on the Faroe Islands. In addition, he describes two other groups of men, which maybe to a lesser extent can be seen as recognised ideals, Lonestars and Glocal pragmatists. Even though Gaini stresses that the typology should be interpreted with caution, he states that it is useful with regards to emphasising some of the changes that the Faroese masculinity ideal is undergoing. He states the following about the different typologies:

Atlantic cowboy represents the young Faroese man who has no or limited education: "Atlantic cowboys [...] are from families primarily engaged in the fisheries on land and offshore and belong to what remains of Faroese working-class [...] many cowboys have large personal incomes acquired from skilled and manual work on industrial trawlers" (Gaini 2006: 47). Among this group of young men, practical experience counts more than education, and some are explicitly anti-intellectual and distance themselves from persons with higher education. Gaini writes that this group is under increasing pressure, and it is particularly losing status in Torshavn, where they are increasingly perceived as being uncultured "cavemen" by other young people (Gaini 2006: 51).

Urban youth is another type of young Faroese men, and even though "urban" is a part of the denomination, they are not necessarily only to be found in Torshavn. "Urban" rather refers to the ideals this group of young men identify themselves with; namely culture and fashion ideals, which are associated with life in Western cities. These young men see themselves more as cosmopolitans than the "simple" cowboys, and they orient themselves more towards the norms of their peers than those of their family, and are often very career oriented and education-minded (Gaini 2006: 51-54).

Lonestars are described by Gaini as "young men leading lives isolated in their media-rich rooms" (Gaini 2005: 54). 
Glocal pragmatists are more opportunistic young Faroese men, who continuously seek new opportunities on the basis of local and global developments - according to Gaini, this group typically consists of young foreigners, and young Faroese men, who have experience from stays abroad.

Gaini emphasises that all types of young men are to be found both in and outside of Torshavn, and that they should not be considered as clearly defined categories, as some young men manage to "shop around" between the different types of male ideals. Yet, overall, it is his assessment that the group of Atlantic cowboys is under increasing pressure, and is becoming marginalised by the Urban youth, which is the group that currently is growing the most in the Faroe Islands. According to Gaini, this is partly due to the increased significance of formal qualifications and educational certificates, and that the young men with no or limited education increasingly will have a hard time gaining a firm foothold on the Faroese labour market - including in the fishing industry.

\subsection{The Åland Islands}

It has not been possible to find any literature regarding changes in masculinity ideals on Åland, and as has been explained above, the gender ideals on Åland to some extent appear stable, and are also not an issue which seems to have attracted scholarly attention. 


\section{List of references}

Abelsen, Birgit. (2005). "Studieområdet - en statistisk presentasjon." I Med periferien i sentrum: - en studie av lokal velferd, arbeidsmarked og kjønnsrelasjoner i den nordiske periferien, redigeret af Anna-Karin Berglund, Susanne Johansson, og Irene Molina, 16-34. Alta: Norut NIBR Finnmark.

Aðalsteinsdóttir, Arnfríður. (2010). "Kvinder og mænd må tale sammen." I Ligestilling i skolen: rapport fra nordiske konferencer i Tórshavn og Reykjavik under Islands formandskab i Nordisk Ministerråd, redigeret af Erla Sigurđardóttir, 43-46. Copenhagen, Denmark: Nordisk Ministerråd. http://oclcmarc.ebrary.com/id/10569943.

Aner, Louise Glerup, og Høgni Kalsø Hansen. (2014). Flytninger fra byer til land- og yderområder: højtuddannede og socialt udsatte gruppers flytninger fra bykommuner til land- og yderkommuner - mønstre og motiver. SFI - Det Nationale Forskningscenter for Velfærd.

Angell, Elisabeth, og Marit Aure. (2011). "Kompetansebehov og rekruttering i fiskeforedlingsindustrien." Norut Alta-Áltá Rapport 2011:1.

Angell, Elisabeth, Marit Aure, Ivar Lie, Vigdis Nygaard, og Toril Ringholm. (2013). Attraktive lokalsamfunn og arbeidsmarkedsregioner i Nord-Norge. Norut Alta-Áltá Rapport 2013:7.

Aure, Marit. (2013). "Highly skilled dependent migrants entering the labour market: Gender and place in skill transfer." Geoforum, Risky natures, natures of risk, 45: 275-84. doi:10.1016/j.geoforum.2012.11.015.

Berglund, Anna-Karin, Susanne Johansson, og Irene Molina. (2005). Med periferien $i$ sentrum: - en studie av lokal velferd, arbeidsmarked og kjønnsrelasjoner i den nordiske periferien. Alta: Norut NIBR Finnmark.

Bjarnason, T., og T. Thorlindsson. (2006). "Should I stay or should I go? Migration expectations among youth in Icelandic fishing and farming communities." Journal of Rural Studies 22 (3): 290-300. doi:10.1016/j.jrurstud.2005.09.004.

Bjerring, Bodil. (2000). "Familieorienterede livsformer og arbejdspladsernes fleksibilitetsønsker: nogle udkanterfaringer." TemaNord, nr. 2000-575: 165-87.

Bjerring, Bodil. (2007). "Skagen. En udkant til tiden." I Landvindinger. Landdistriktsforskning og -perspektiver., Gunnar Svendsen og Hanne Wittorff Tanvig. Odense: Syddansk Universitetsforlag.

Bjørnholt, Margunn. (2014). "Changing Men, Changing Times - Father's and Sons from an Experimental Gender Equality Study." The Sociological Review 62 (2): 295315. doi:10.1111/1467-954X.12156.

Bloksgaard, Lotte. (2011). "Masculinities, Femininities and Work - The Horizontal Gender Segregation in the Danish Labour Market." Nordic Journal of Working Life Studies 1 (2): pp. 5-21.

Bloksgaard, Lotte, Stine Thidemann Faber, og Claus D. Hansen. (2013). "Drenge i Udkanten - Køn, Stedtilknytning og Uddannelse." I Drenge og maskuliniteter i ungdomsuddannelserne, redigeret af Christian Helms Jørgensen, 1. udg., 87-208. Roskilde Universitetsforlag. 
Boolsen, Merete Watt. (2008). Uddannelsesplanen: Rapport 1. Det Grønlandske Uddannelsesdepartementet.

Boolsen, Merete Watt. (2009). Uddannelsesplanen: Rapport Nr. 2. Det Grønlandske Uddannelsesdepartementet.

Boolsen, Merete Watt. (2010a). "Pixi: Den Gode Skole." Det Grønlandske Uddannelsesdepartement.

Boolsen, Merete Watt. (2010b). Uddannelsesplanen: Rapport 3. Det Grønlandske Uddannelsesdepartementet - Det Grønlandske Selvstyre.

Boolsen, Merete Watt. (2010c). Unge i Uddannelse og Arbejde. Notat til KIIN. Det Grønlandsek Uddannelsesdepartementet/-ministeriet.

Boolsen, Merete Watt. (2013). "Evaluating Education in Greenland. How is Power Exercised through Evaluation Models?." Scandinavian Journal of Public Administration 16 (3): 65-82. http://130.241.16.45/ojs/index.php/sjpa/article/view/1707.

Bourn, Douglas. (2008). "Young People, Identity and Living in a Global Society." Policy \& Practice: A Development Education Review, nr. 7: 48-61.

Brannen, Julia, og Ann Nilsen. (2002). "Young People's Time Perspectives: From Youth to Adulthood." Sociology 36 (3): 513-37. doi:10.1177/0038038502036003002.

Budtz, Camilla, Ingelise Olesen, og Cecilia P. Pedersen. (2011). "De unges udsagn om det gode børne- og ungdomsliv." I Det Svære Ungdomsliv: Unges Trivsel i Grønland 2011 - en undersøgelse blandt de ældste folkeskoleeleve, redigeret af Cecilia Petrine Pedersen og Peter Bjerregaard. København: Statens Institut for Folkesundhed; Naalakkersuisut.

Bye, Linda Marie. (2009). "'How to Be a Rural Man": Young Men's Performances and Negotiations of Rural Masculinities." Journal of Rural Studies 25 (3): 278-88. doi:10.1016/j.jrurstud.2009.03.002.

Bye, Linda Marie. (2010). "Bygdas unge menn: En studie av bygdemenns forhandlinger om og utforming av rurale maskuliniteter." Geografisk Institutt: Norges Teknisk- naturvidenskabelige Universitet.

Bæck, Unn Doris. (2004). "The Urban Ethos Locality and Youth in North Norway." Young 12 (2): 99-115. doi:10.1177/1103308804039634.

Bæck, Unn Doris. (2012). "Refleksjoner om nordnorske utdanningsvalg. (Reflections on educational choices in Northern Norway)." I Hvor går Nord-Norge. Et institusjonelt perspektiv på folk og landsdel (An institutional perspective on people and places in Northern Norway)., Svein Jentoft, Jens-Ivar Nergård, og Kjell Arne Rørvik, 117-26. Stamsund: Orkana Akademisk.

Bæck, Unn-Doris Karlsen, og Gry Paulgaard, red. (2012). Rural futures?: finding one's place within changing labour markets. Stamsund: Orkana Akademisk.

Bærenholdt, Jørgen Ole, og Brynhild Granås, red. (2008). Mobility and Place. Enacting Northern European Peripheries. Aldershot: Ashgate.

Båtevik, Finn Ove. (2001). "Ung i utkant - tanker om framtida blant ungdom frå utkantkommunar.", Tidsskrift for ungdomsforskning, 1 (1): 90-103.

Carson, Professor Dean, Andrew Taylor, Professor Lee Huskey, Professor Prescott C. Ensign, og Assoc Prof Rasmus Ole Rasmussen. (2012). Demography at the Edge: Remote Human Populations in Developed Nations. Ashgate Publishing, Ltd.

Christensen, Else. (2013). Ung i det Grønlandske samfund. København: SFI - Det Nationale Forskningscenter for Velfærd.

Christensen, Else, Lise G. Kristensen, og Siddhartha Baviskar. (2008). Børn i Grønland: en kortlægning af 0-14-årige børns og familiers trivsel. København: Nationale forskningscenter for velfærd. 
Christensen, Sascha, og Helene Nielsen Pristed. (2013)."Udkantsdanmark: Avisernes (med)produktion af Nordjyllands territorielle stigma." Praktiske Grunde, nr. 3: 520. http://praktiskegrunde.dk/praktiskegrunde3-2013-samlet.pdf.

Connell, R.W. (1995). Masculinities. University of California Press.

Corbett, Michael. (2007). Learning to leave. The Irony of Schooling in a Coastal Community. Fernwood Publishing.

Cresswell, Tim. (1996). In Place/Out of Place: Geography, Ideology and Transgression. Minnesota Press.

Cuba, Lee, og David M. Hummon. (1993). "A Place to Call Home: Identification with Dwelling, Community, and Region." The Sociological Quarterly 34 (1): 111-31. http://www.jstor.org/stable/4121561.

Curtis, Tine, Helmer B. Larsen, Karin Helweg-Larsen, Cecilia P. Pedersen, Ingelise Olesen, Kristian Sørensen, Marit E. Jørgensen, og Peter Bjerregaard. (2006). Unges trivsel i Grønland. Arktisk Forskningsjournal. Nuuk: Forlaget Atuagkat ApS.

Dahlgren, Anita. (1997). "Reviews : Peter Waara Ungdom I Gränsland (Youth on the Border) Umeå: Borea Bokförlag, 1996." Young 5 (2): 71-72. doi:10.1177/ 110330889700500206.

Dahlström, Margareta. (1996). "Young women in a male periphery - Experiences from the Scandinavian north." Journal of Rural Studies 12 (3): 259-71. doi:10.1016/0743-0167(96)00018-6.

Dahlström, Margareta, Andra Aldea-Partanen, Katarina Fellman, Nino J. Larsen, Hjalti Jóhannesson, Jesper Manniche, Grethe M. Olsen, og Tage Petersen. (2006). How to Make a Living in Insular Areas - Six Nordic Cases. Stockholm: Nordregio.

Dalin, Rolf, og Göran Bostedt. (2010). Ung i Västernorrland: en uppföljning av ungdomspolitiken. Redigeret af FoU Västernorrland. Härnösand: FoU Västernorrland.

Danske Regioner. (2010). Vækst i hele Danmark. Danske Regioner.

Ebbensgaard, Aase HB, og Steen Beck. (2009). Mellem Bagenkop og Harboøre - elevfortællinger fra danske udkanter. Gymnasiepædagogik, nr. 75, 2009. Institut for Filosofi, Pædagogik og Religionsstudier, Syddansk Universitet.

Edvardsdóttir, Anna Guhrún Gudrun. (2013). "Place and space for women in a rural area in Iceland." Education in the North, nr. 20 (special issue): 73-89.

Eistrup, Jette, og Wolfgang Kahlig. (2005). "Historiske forandringer på ligestillingsområdet i Grønland som faktor for velfærdsudvikling." I Med periferien i sentrum: - en studie av lokal velferd, arbeidsmarked og kjønnsrelasjoner i den nordiske periferien, redigeret af Anna-Karin Berglund, Susanne Johansson, og Irene Molina, 201-14. Alta: Norut NIBR Finnmark.

Ellingsen, Dag, og Ulla-Britt Lilleaas. (2010). ”Det gode liv på Sørlandet og tradisjonelle kjønnsroller." Universitetet i Agder: Senter for likestilling.

Eriksson, Madeleine. (2010). "(Re)producing a "peripheral" region-Northern Sweden in the news." Geografiska Annaler: Series B, Human Geography 90 (4): 369-88.

Eriksson, Madeleine, Helene Pristed Nielsen, og Gry Paulgaard. (2015). "The Internal Other: Reproducing and Reworking Center and Periphery." I Remapping Gender, Place and Mobility - Global Confluences and Local Particularities in Nordic Peripheries, Stine Thidemann Faber og Helene Pristed Nielsen, 1. udg., 37-54. Surry: Ashgate Publishing, Ltd.

Eriksson, Madeleine, og Aina Tollefsen. (2015). "New Figurations of Labour in Gendered Global Circuits: Migrant Workers in the Forest Berry Industry in Norrland, Sweden." I Remapping Gender, Place and Mobility - Global Confluences and Local Particularities in Nordic Peripheries, Stine Thidemann Faber og Helene Pristed Nielsen, 1. udg., 127-42. Surry: Ashgate Publishing, Ltd. 
Faber, Stine Thidemann, Claus D. Hansen, Lotte Bloksgaard, og Karina Torp. (2014). Unge på Kanten - køn, lokalitet og uddannelse. Aalborg Universitet.

Faber, Stine Thidemann, og Helene Pristed Nielsen. (2015a). "Centering the Periphery." I Remapping Gender, Place and Mobility - Global Confluences and Local Particularities in Nordic Peripheries, Stine Thidemann Faber og Helene Pristed Nielsen, 1. udg., 3-18. Surry: Ashgate Publishing, Ltd.

Faber, Stine Thidemann, og Helene Pristed Nielsen. (2015b). "At Home Away? Particularities of Belonging seen through a Camera Lens." I Remapping Gender, Place and Mobility - Global Confluences and Local Particularities in Nordic Peripheries, Stine Thidemann Faber og Helene Pristed Nielsen, 1. udg., 73-92. Surry: Ashgate Publishing, Ltd.

Forsberg, Gunnel. (1997). "Rulltrapperegioner och social infrastruktur." I Om makt och kön: i spåren av offentliga organisationers omvandling : rapport till Utredningen om fördelningen av ekonomisk makt och ekonomiska resurser mellan kvinnor och män., Elisabeth Sundin. Arbetsmarknadsdepartementet.

Forsberg, Gunnel. (2001). "The difference that space makes: A way to describe the construction of local and regional gender contracts." Norsk Geografisk Tidsskrift 55 (3): 161-65.

Forsberg, Gunnel. (2002). "En feministisk exkursion i ett vetenskapligt landskap." I Svensk kulturgeografi och feminism - rötter och rörelser i en rumslig disciplin, K. Schough. Karlstad University Studies: Universitetstryckeriet.

Forsberg, Gunnel, og Susanne Stenbacka. (2013). "Mapping Gendered Ruralities." European Countryside 5 (1): 1-20.

Fossland, Trine, og Marit Aure. (2011). "Når høyere utdanning ikke er nok: Integrasjon av høyt utdannede innvandrere på arbeidsmarkedet." Sosiologisk tidsskrift 19 (02): 131-52.

Friberg, Tora. (2000). "Vad göra för att få kvinnorna att bo kvar?." I Vi flytt nu. Om befolkningsflyttningar i Sverige., G. Graninger, G. Blücher, og L. Nyström. Skrift XIV 2000. Stiftelsen Vadstena Forum för samhällsbyggande.: Stadsmiljörådet \& Vadstena Forum.

Fuglestad, Otto L. (2002). "Reviews: Gry Paulgaard: Ungdom, Lokalitet Og Modernitet (Youth, Identity and Locality) A Doctoral Dissertation on Cultural Encounters and Identity Construction in a Coastal Community in Northern Norway. University of Tromsø: Tromso, 2000." Young 10 (3-4): 128-30. doi:10.1177/ 110330880201000309.

Gaini, Firouz. (2003). Ung í Føroyum. Námsrit 1/2003. Klaksvik: Granskingardepilin fyri Samfelagsmenning.

(2005). "Faroese Youth Preferences of Education, Work and Leisure." I Youth - Similarities, Differences, Inequalities, redigeret af Vesa Puuronen, Jarna SoilevuoGrønnerød, og Jatta Herranen, 13-14. Karelian Institute 1. Kirjasto: University of Joensuu - Joensunn Yliopiston.

Gaini, Firouz. (2006). Once Were Men. Maculinities among young men in the Faroe Islands. In Fróðskaparrit 54 (Annales Societatis Scientiarum Færoensis). Torshavn: Faroese University Press.

Gaini, Firouz. (2010). "Hvad har du lært i skolen i dag? Familiekapital og skole på Færøerne." Norsk senter for barneforskning, nr. 3: 7-29.

Gaini, Firouz. (2011a). "Fortidens helte i modvind." NIIK - Tema 1996-2011 - Män och maskuliniteter. 
Gaini, Firouz. (2011b). "The adversity of Heroes of the past - Masculinity and Identity among young men in the Faroe Islands." I Among the Islanders of the North - An anthropology of the Faroe Islands, redigeret af Firouz Gaini, 163-99. Torshavn: Faroe University Press.

Gerrard, Siri. (2013). "Mobilities, materialities, and masculinities: Interconnected mobility practices in Norwegian coastal fisheries." Norsk Geografisk Tidsskrift - Norwegian Journal of Geography 67 (5): 312-19. doi:10.1080/00291951.2013.847482.

Giddens, Anthony. (1991). Modernity and Self Identity. Self and Society in the Late Modern Age. Cambridge: Polity Press.

Giskeødegård, Marte Fanneløb, og Gro Marit Grimsrud. (2014). Kjønnsperspektiv på rekruttering av innbyggjarar til Møre og Romsdal. 52. Møreforskning, Højskulen i Volda, Møre og Romsdal Fylkeskommune.

Gislason, Ingólfur. (2011). "Isländska män i kvinnoyrken." I Nordiske mænd til omsorgsarbejde!: - en forskningsbaseret erfaringsomsamling på initiativer til at rekruttere, uddanne og fastholde mænd efter finanskrisen, redigeret af Steen Baagøe Nielsen, 248-59. Roskilde: VELPRO - Center for Velfærd, Profession og Hverdagsliv.

Grimsrud, Gro Marit. (2011). "Gendered Spaces on Trial: The Influence of Regional Gender Contracts on in-Migration of Women to Rural Norway." Geografiska Annaler: Series B, Human Geography 93 (1): 3-20. doi:10.1111/j.1468-0467.2011.00358.x.

Grimsrud, Gro Marit, og Marit Aure. (2013). Tilflytting for en hver pris? En studie av tilflyttingsarbeid i norske distriktskommuner. Møreforsking, Norut og Ideas2Evidence - Distriktssenteret.

Gunnarsson, Yvonne. (1993). Träffpunkt för tjejer. Rapport från ett projekt med tonårsflickor på landsbygden. 5. Uppsala: Sveriges Lantbruksuniversitet.

Gunnarsson, Yvonne. (1994). "Livet på landet - sett ur tonårsflickors perspektiv." I Ungdomskultur i Sverige., Johan Fornäs, Ulf Boëthius, Michael Forsman, Hillevi Ganetz, og Bo Reimer, 105-24. FUS-rapport nr 6. Stockholm/Stehag: Brutus Östlings Bokförlag Symposion AB 1994.

Gustafsson, Jan, og Elisabet Öhrn. (2012). "Gender, Achivement and Place: Boys and Masculinities in a Rural Area." I Paper presented at Aare 2012, Sydney 2-6 December.

Hamilton, Lawrence C., og Oddmund Otterstad. (1998). "Sex Ratio and Community Size: Notes from the Northern Atlantic." Population and Environment 20 (1): 11-22 doi:10.1023/A:1023347817418.

Hamilton, Lawrence C., og Rasmus Ole Rasmussen. (2010). "Population, Sex Ratios and Development in Greenland." Arctic 63 (1): 43-52.

Hammer, Torild. (1999). "The Influence of Different Compensation Levels of Unemployment Benefits on Job Chances among Unemployed Youth: A Comparative Study of the Nordic Countries." Acta Sociologica 42 (2): 123-34. doi:10.1177/ 000169939904200202.

Hansen, Anne Merrild, og Pelle Tejsner. (2015). "Challenges and opportunities for residents in the Upernavik District" (under udgivelse).

Hansen, Klaus Georg, Rasmus Ole Rasmussen, og Ryan Weber, red. (2013). "Proceedings from the First International Conference on Urbanisation in the Arctic." I Nordregio Working Paper 2013:6.

Hansen, Sune Jon. (2011). "Om fisk, familie og f $\backslash$ a erdigheder. Udpluk fra et etnografisk studie af relationerne mellem lokalitet, erhverv og uddannelse." Af Praktiske Grunde, $n r, 77-86$. 
Hansen, Sune Jon. (2014). "At komme fra "udkanten" Om sted, stedsans og unges uddannelsesstrategier i et socialt rum under forandring - belyst gennem et casestudie af Hirtshals, en havneby på den nordjyske vestkyst." Ph.D.-afhandling, Institut for Medier, Erkendelse og Formidling, Københavns Universitet.

Hedetoft, Anders, og Irena Stefaniak. (2014). Ungdomsuddannelser med fokus på yderkommuner. Center for Regional- og turismeforskning.

Heggen, Kåre. (2002). "Utkantjentene sin stille revolusjon." Tidsskrift for ungdomsforskning 2 (2): 3-20.

Heijstra, Thamar M., Pat O’Connor, og Gudbjörg Linda Rafnsdóttir. (2013). "Explaining gender inequality in Iceland: what makes the difference?." European Journal of Higher Education 3 (4): 324-41. doi:10.1080/21568235.2013.797658.

Helvé, Helena. (2003). "Ung i Utkant - Aktuel forskning glesbygdsungdomar i Norden." I Ung i Utkant - Aktuel forskning glesbygdsungdomar i Norden., redigeret af Helena Helvé, 187-223. København: Nordisk Ministerråd.

Hermansen, Jonathan. (2011). "Udkanten: hvorfor kragerne vender - En undersøgelse af unge mænds tilknytning til Udkantsdanmark." Speciale, Københavns Universitet.

Hermansen, Jonathan, og Sara Lea Rosenmeier. (2011). Hvorfor bliver unge mænd boende på landet? En undersøgelse blandt unge mænd på Horne Land. Rapporten er udarbejdet af Rådgivende Sociologer ApS.

Hoven, Bettina V., og Kathrin Hörselmann. (2005). Spaces of Masculinities. London: Routledge.

Hovgaard, Gestur. (2015). "Being Away; Being Home; Being Both: The Case of Faroese Maritime Workers." I Remapping Gender, Place and Mobility - Global Confluences and Local Particularities in Nordic Peripheries, Stine Thidemann Faber og Helene Pristed Nielsen, 1. udg., 175-90. Surry: Ashgate Publishing, Ltd.

Hovgaard, Gestur, Grétar Thór Eythórsson, og Katarina Fellman. (2004). Future Challenges to Small Municipalities: The Cases of Iceland, Faroe Islands and Åland Islands. Stockholm: Nordregio, Nordic Centre for Spatial Development.

Hovgaard, Gestur, og Sámal M. Kristiansen. (2008). "Villages on the Move: From Places of Necessity to Places of Choice." I Mobility and Place : Enacting Northern European Peripheries, redigeret af Jørgen Ole Bærenholdt og Brynhild Granås, 6174. Abingdon, Oxon: Ashgate Publishing Group.

Howard, Judith A. (2000). "Social Psychology of Identities." Annual Review of Sociology 26: 367-93.

Hudson, Christine, og Malin Rönnblom. (2007). "Regional Development Policies and the Constructions of Gender Equality: The Swedish Case." European Journal of Political Research 46 (1): 47-68. doi:10.1111/j.1475-6765.2006.00642.x.

Høgnesen, Runa Preeti. (2015). "Cross-Border Marriage, Community and Construction of Womanhood - An Analysis of Married Philippine and Thai Women in the Faroe Islands and their Experiences with Inclusion and Exclusion." Endnu ikke påbegyndt Ph.d., Fróðskaparsetur Føroyar.

Haagensen, Klaus M. (2014). Nordic Statistical Yearbook 2014. 52. København: Statistics Denmark - Nordic Council of Ministers.

Jacobsen, Bjørn, og Beinta Jákupsstova. (2005). "Kvinnerollen på Færøyene i endring - en sammenlikning av kvinners politiske medborgerskap i to kommuner." I Med periferien i sentrum: - en studie av lokal velferd, arbeidsmarked og kjønnsrelasjoner $i$ den nordiske periferien, redigeret af Anna-Karin Berglund, Susanne Johansson, og Irene Molina, 169-200. Alta: Norut NIBR Finnmark.

Jafnréttisstofa - Centre for gender Equality Iceland. (2012). "Gender Equality in iceland: Information on Gender Equality Issues in Iceland." Jafnréttisstofa. 
Jafnréttisstofa - Centre for Gender Equality Iceland. (2014). "Jafnréttisstofa - Centre for Gender Equality Iceland: Statictics on Women and Men in Iceland 2014."

Jákupsstova, Beinta i, Hans Andrias Sølvará, og Gestur Hovgaard. (2014). Vestnorden - Nye Roller i det Internationale Samfund. Tórshavn: Faroe University Press.

Jensen, Sune Qvotrup, og Ann-Dorte Christensen. (2012). "Territorial stigmatization and local belonging." City: analysis of urban trends, culture, theory, policy, action 16 (1-2): 74-92. doi:10.1080/13604813.2012.663556.

Johansen, Susanne, Susanne Stenbacka, og Marie Nordfeldt. (2005). "Global påverkan och lokala strategier - studier av välfärd och genusrelationer i förändring i Jokkmokk och Pajala." I Med periferien i sentrum: - en studie av lokal velferd, arbeidsmarked og kjønnsrelasjoner i den nordiske periferien, redigeret af Anna-Karin Berglund, Susanne Johansson, og Irene Molina, 35-77. Alta: Norut NIBR Finnmark.

Johansson, Mats, og Daniel Rauhut. (2012). Case study report: Västernorrland's county. Semigra.

Júlíusdóttir, Magnfríður, Unnur Dís Skaptadóttir, og Anna Karlsdóttir. (2013). "Gendered migration in turbulent times in Iceland." Norsk Geografisk Tidsskrift - Norwegian Journal of Geography 67 (5): 266-75. doi:10.1080/00291951.2013.847483.

Kalaoja, Esko, og Janne Pietarinen. (2009). "Small rural primary schools in Finland: A pedagogically valuable part of the school network." International Journal of Educational Research, Reviews of research on rural schools and their communities in British and Nordic countries, 48 (2): 109-16. doi:10.1016/j.ijer.2009.02.003.

Kaldal, Inger. (2000). "Skog, arbeid og dagligliv i kvinners og menns fortellinger fra Trysil og NordVärmland etter 1930." I Skogsliv: Kulturella processer i nordiska skogsbygder, E. J. Kaldal, B. Fritzbøger, og H. Snellman, 85-117. Lund: Historiska Media.

Karlsdóttir, Anna, og Auđur H. Ingólfsdóttir. (2011). "Gendered Outcomes of Socioeconomic Restructuring: A Tale from a Rural Village in Iceland." NORA - Nordic Journal of Feminist and Gender Research 19 (3): 163-80. doi:10.1080/ 08038740.2011 .594029$.

Karlsen, Unni D. (2001). "Ung i spenningens land. Analyser af preferanser knyttet til valg av utdanning, yrke og bosted blandt nordnorsk ungdom." Ph.d-afhandling, Tromsø: University of Tromsø.

Kelly, Ursula A. (2009). "Learning to Lose: Rurality, Transience, and Belonging (A Companion to Michael Corbett)." Journal of Research in Rural Education (Online) 24 (11): 1.

Kenway, Jane, og Anna Hickey-Moody. (2006). Masculinity beyond the Metropolis. Hampshire: Palgrave Macmillan.

Keskitalo-Foley, Seija. (2013). "Highly Educated Women in Lapland Looking for Agency in the Neoliberal Era." I Northern Insights: Feminist Inquiries into Politics of Place, Knowledge and Agency, redigeret af Seija Keskitalo-Foley, Päivi Naskali, og Pälvi Rantala, 130-52. Rovaniemi: Lapland University Press.

Knudsen, Karin J. L. (2009). "Køn og magt i politik og erhvervsliv på Færøerne.” I Kön och makt i Norden. Del 1: Landsrapporter, redigeret af Kristi Niskanen og Anita Nyberg, 321-41. TemaNord 2009:569. København: Nordisk Ministerråd.

Knudsen, Karin J. L. (2010). "Kvinder og velfærd på Færøerne." I Kvinder og Velfærd i Vestnorden, redigeret af Guðbjörg Linda Rafnsdóttir, 13-37. TemaNord 2010:578. København: Nordisk Ministerråd.

Kocziszky, György, Zoltan Nagy, Daniel Rauhut, og Ildikó Györffy. (2012). "Gender related out-migration tendencies in Northern Hungary and in Västernorrland, Sweden." Paper presented at the Regional Studies Association's Conference, 13th16th May 2012 in Delft, the Netherlands. 
Larsen, Jesper F.H., og Anne-Mette Hjalager. (2012). Uddannelse og yderområder. 17. Center for Landdistriktsforskning.

Larsen, Lene, og Signe Hvid Thingstrup. (2014). Rum for mænd?: - en vidensopsamling om drenge og mænd i uddannelse. Roskilde: Roskilde Universitet.

Lindberg, Malin. (2014). "From Exclusion to Inclusion in Public Innovation Support? Innovative Practices in Bottom-up Networks." Scandinavian Journal of Public Administration 18 (4): 91-107.

Lindberg, Malin, og Maria Udén. (2010). "Women, reindeer herding and the Internet: an innovative process in northern Sweden." Innovation: The European Journal of Social Science Research 23 (2): 169-77. doi:10.1080/13511610.2010.512441.

Lundholm, Emma, Jörgen Garvill, Gunnar Malmberg, og Kerstin Westin. (2004). "Forced or Free Movers? The Motives, Voluntariness and Selectivity of Interregional Migration in the Nordic Countries." Population, Space and Place 10 (1): 59-72. doi:10.1002/psp.315.

Massey, Doreen. (1994). Space, Place and Gender. Cambridge: Polity Press.

Massey, Doreen. (2005). For Space. London: SAGE Publication.

McDowell, Linda. (2003). Redundant Masculinities? Employment change and white working class youth. Oxford: Blackwell Publishing.

Medonos, Jakub C. (2010). "Ungdom og urbanitet i Grønland”,. Ph.d-afhandling, København: Københavns Universitet: Det Humanistiske Fakultet.

Munkejord, Mai Camilla. (2006). "Challenging Discourses on Rurality: Women and Men In-Migrants' Constructions of the Good Life in a Rural Town in Northern Norway." Sociologia Ruralis 46 (3): 241-57. doi:10.1111/j.1467-9523.2006.00415.x.

Munkejord, Mai Camilla. (2011). Hjemme i nord. Stamsund: Orkana Akademisk.

Nielsen Baagøe, Steen. (2011). Nordiske mænd til omsorgsarbejde!: - en forskningsbaseret erfaringsomsamling på initiativer til at rekruttere, uddanne og fastholde mænd efter finanskrisen. Roskilde: VELPRO - Center for Velfærd, Profession og Hverdagsliv.

Nielsen Baagøe, Steen, og Christian Helms Jørgensen. (2013). "Drenges vilkår og veje i uddannelserne." I Drenge og maskuliniteter i ungdomsuddannelserne, redigeret af Christian Helms Jørgensen, 1. udg., 9-23. Roskilde Universitetsforlag.

Ní Laoire, C., og S. Fielding. (2006). "Rooted and routed masculinities. Among the Rural Youth of North Cork and Upper Swaledale." I Country Boys. Masculinity and Rural Life., H. Campell, M. Bell Mayerfield, og M. Finney, 105-19. Pennsylvania: The Pennsylvania State University Press.

Nilsson, Bo, og Anna Sofia Lundgren. (2015). "Logics of rurality: Political rhetoric about the Swedish North." Journal of Rural Studies 37: 85-95. doi:10.1016/j.jrurstud.2014.11.012.

Niskanen, Kirsti, red. (2010). Kön och makt i Norden. 2: Sammanfattande diskussion och analys. TemaNord 2009:569. København: Nordisk Ministerråd.

Nordic Information on Gender - NIKK. (2014). "Åland Islands." Nordic Information on Gender - NIKK.

Nordregio. (2010). Mobilitet I Grønland: Sammenfattende analyse. Stockholm: Nordic Council of Ministers Research Programme.

Paulgaard, Gry. (2002). "Local Identities in a "Globalised World."' Young 10 (3-4): 95-107. doi:10.1177/110330880201000307.

Paulgaard, Gry. (2006). "Identitetskonstruksjoner - hvor langt rekker de." Tidsskrift for ungdomsforskning 6 (1): 67-88. 
Paulgaard, Gry. (2012). "Geography of opportunity. Approaching adulthood at the margins of the northern European periphery." I Rural futures?: finding one's place within changing labour markets, redigeret af Unn-Doris Karlsen Bæck og Gry Paulgaard, 189-216. Stamsund: Orkana Akademisk.

Paulgaard, Gry. (2015). "Place Attatchment, Unemployment and Masculinity: Young Men in the High North." I Remapping Gender, Place and Mobility - Global Confluences and Local Particularities in Nordic Peripheries, Stine Thidemann Faber og Helene Pristed Nielsen, 1. udg., 207-20. Surry: Ashgate Publishing, Ltd.

Pedersen, Cecilia P., og Peter Bjerregaard, red. (2011). "Det Svære Ungdomsliv: Unges Trivsel i Grønland 2011 - en undersøgelse blandt de ældste folkeskoleeleve." I Det Svære Ungdomsliv: Unges Trivsel i Grønland 2011 - en undersøgelse blandt de ældste folkeskoleeleve. København: Statens Institut for Folkesundhed; Naalakkersuisut.

Pedersen, Paul. (2013). "Applying longitudinal statistics in migration analysis: A case study of migration among young adults in Northern Norway." Norsk Geografisk Tidsskrift - Norwegian Journal of Geography 67 (5): 295-303. doi:10.1080/00291951.2013.847853.

Peltomaa, Hanna. (2013). "The Only Gay in the Village? Lesbians, Gay Men and Bisexuals Living in Rural Communities in Finnish Lapland." I Northern Insights: Feminist Inquiries into Politics of Place, Knowledge and Agency, redigeret af Seija Keskitalo-Foley, Päivi Naskali, og Pälvi Rantala, 153-73. Rovaniemi: Lapland University Press.

Petridou, Evangelia. (2011). "Theorizing public entrepreneurs: women and men of innovative action." Paper presented at the ECPR conference in Reykjavik, Iceland in August, 2011.

Pettersson, Katarina. (2012). "Support for Women's Entrepreneurship: A Nordic Spectrum." International Journal of Gender and Entrepreneurship 4 (1): 4-19. doi:http://dx.doi.org/10.1108/17566261211202954.

Pettersson, Örjan. (2002). Socio-economic dynamics in sparse regional structures. Gerum 2002:2. Umeå: Kulturgeografiska institutionen.

Plambech, Sine. (2005). "'Postordrebrude" i Nordvestjylland: transnationale ægteskaber i et omsorgsøkonomisk perspektiv." Dansk Sociologi 16 (1): 91-110.

Poppel, MarieKathrine. (2010). "Kvinder og Velfærd i Grønland." I Kvinder og Velfærd i Vestnorden, redigeret af Guðbjörg Linda Rafnsdóttir, 38-68. TemaNord 578. København: Nordisk Ministerråd.

Poppel, MarieKathrine, og Janus Chemnitz Kleist. (2009). "Køn og magt i politik og erhvervsliv i Grønland." I Kön och makt i Norden. Del 1: Landsrapporter, redigeret af Kristi Niskanen og Anita Nyberg, 341-58. TemaNord 2009:569. København: Nordisk Ministerråd.

Paakkunainen, Kari. (2002). "Peripheral Youth in Hard Marginal and Reflective Social Policy: A Companionship Model for the Barents Region from Finnish Kainuu." Young 10 (3-4): 61-80. doi:10.1177/110330880201000305.

Rafnsdóttir, Gudbjörg Linda, og Ingibjörg Lilja Ómarsdóttir. (2010). "Kvinder og velfærd i Island." I Kvinder og Velfærd i Vestnorden, redigeret af Guðbjörg Linda Rafnsdóttir, 69-97. TemaNord 578. København: Nordisk Ministerråd.

Rasmussen, Rasmus Ole. (2005). Analyse af fangererhvervet i Grønland. Direktoratet for Miljø og Natur, Direktoratet for Fiskeri og Fangst, KNAPK, KANUKOKA samt Grønlands Statistik.

Rasmussen, Rasmus Ole. (2009). "Gender and Generation: Perspectives on Ongoing Social and Environmental Changes in the Arctic." Signs 34 (3): 524-32.

doi:10.1086/593342. 
Rasmussen, Rasmus Ole, Johanna Roto, Lise Smed Olsen, og Lisbeth Greve Harbo. (2011). "Status for bosteder i Grønland med særlig fokus på bygderne." Redigeret af Nordregio. Nordic Council of Ministers Research Programme Report 2010.

Rauhut, Daniel, og Mats Johansson. (2012). "'Should I Stay or Should I go?"-A Case Study on Young Women Leaving Rural Sweden." I ERSA conference papers. European Regional Science Association.

Rauhut, Daniel, og Helene Littke. (2014). "“'A one way ticket to the city, please!” On young women leaving the Swedish peripheral region Västernorrland."” A paper presented at the Regional Studies Association's European Conference 15-18 June 2014, Izmir, Turkey.

Rauhut, Daniel, Rasmus O. Rasmussen, Johanna Roto, Per Francke, og Sara Östberg. (2008). The Demographic Challenge to the Nordic Countries. Nordregio Working Paper 2008 1. Stockholm: Nordregio.

Ravenstein, Ernst Georg. (1885). "The Laws of Migration." Journal of Statistical Society, nr. 48: 167-227.

Reisby, Kirsten, og Susanne V. Knudsen. (2005). Køn, ligestilling og skole 1990-2004. NIKK-Rapport. Nordisk Ministerråd.

Rennie, Frank L., Sigurbjörg Jóhannesdóttir, og Stefania Kristinsdottir. (2011). "ReThinking Sustainable Education Systems in Iceland: The Net-University Project." nternational Review of Research in Open and Distance Learning 12 (4).

Roalsø, Kari Mette. (1997). Ungdoms levekår i 1990-årene. Oslo/Kongsvinger: Statistisk Sentralbyå.

Rye, Johan Fredrik. (2006). "Heading for the Cities? Gender and Lifestyle Patterns in Rural Youths' Residential Preferences." Norwegian Journal of Geography 60 (3): 199-208. doi:10.1080/00291950600889988.

Rye, Johan Fredrik. (2007). "Kjønnsperspektiv på flytteforskning om ungdom." Barn - Norsk senter for barneforskning, nr. 1: 81-101.

Rygaard, Jette. (2003). "Youth Culture,Media and Globalization Processes in Greenland." Young 11 (4): 291-308. doi:10.1177/11033088030114001.

Rygaard, Jette, og Birgitte Kleist Pedersen. (2003). "Grønlandske Unge mellem Tradition og Globalisering." I Ung i Utkant - Aktuel forskning glesbygdsungdomar i Norden., redigeret af Helena Helvé, 272-98. København: Nordisk Ministerråd.

Skaptadóttir, Unnur D. (2015). "Women and Men on the Move: From the Philipphines to Iceland." I Remapping Gender, Place and Mobility - Global Confluences and Local Particularities in Nordic Peripheries, Stine Thidemann Faber og Helene Pristed Nielsen, 1. udg., 143-58. Surry: Ashgate Publishing, Ltd.

Skaptadóttir, Unnur Dís, og Anna Wojtynska. (2008). "Labour Migrants Negotiating Places and Engagements." I Mobility and Place : Enacting Northern European Peripheries, redigeret af Jørgen Ole Bærenholdt og Brynhild Granås, 115-26. Abingdon, Oxon: Ashgate Publishing Group.

Skaptadttir, Unnur Ds. (2004). "Responses to Global Transformations: Gender and Ethnicity in Resource-Based Localities in Iceland." Polar Record 40 (3): 261-67. doi:10.1017/S0032247404003663.

Skarpenes, Ove, og Ann Christin E. Nilsen. (2014). "Regional Gender Inequality in the Norwegian Culture of Equality." Gender Issues 32 (1): 39-56. doi:10.1007/s12147014-9131-0.

Skrede, Kari. (2004). "Familiepolitikkens grense. Ved "likestilling light"?." I Velferdsstaten og Familien. Utfordringer og Dilemmaer., Anne Lise Ellingsæter og Leira Arnlaug. Oslo: Gyldendal Norsk Akademisk. 
Stenbacka, Susanne. (2007). "Rural Identities in Transition: Male Unemployment and Everyday Practice in Northern Sweden." I Research in Rural Sociology and Development, 13:83-111. Bingley: Emerald (MCB UP).

Stenbacka, Susanne. (2011). "Othering the rural: About the construction of rural masculinities and the unspoken urban hegemonic ideal in Swedish media." Journal of Rural Studies 27 (3): 235-44. doi:10.1016/j.jrurstud.2011.05.006.

Styrkársdóttir, Auður. (2009). "Kön och makt i isländsk politik." I Kön och makt $i$ Norden. Del 1: Landsrapporter, redigeret af Kristi Niskanen og Anita Nyberg, 13158. TemaNord 2009:569. København: Nordisk Ministerråd.

Sundin, Elisabeth. (2010). "Gender mainstreaning in innovation systems and triple helix constellations." I. Helix Excellence Centre and Department of Business Administration. Linköping University, Sweden.

Svendsen, Gunnar. (2007). "There's More to the Picture than Meets the Eye. Udnyttelse af stedbundne ressourcer i danske udkantssamfund." I Landvindinger. Landdistriktsforskning og -perspektiver. Gunnar Svendsen og Hanne Wittorff Tanvig. Odense: Syddansk Universitetsforlag.

Svensson, Lotta. (2006). "Vinna och försvinna: drivkrafter bakom ungdomars utflyttning från mindre orter." Linköping: Linköpings Universitet, Filosofiska fakulteten.

Sørlie, Kjetil, Marit Aure, og Bjørg Langset. (2012). Hvorfor flytte? Hvorfor bli boende?: bo- og flyttemotiver de første årene på 2000-tallet. Oslo: Norsk institutt for byog regionforskning.

Tanvig, Hanne Wittorff. (2010). Kvindelige iværksættere i landdistrikter - ikke bare en bitte biks. 124. Arbejdsrapport Skov \& Landskab, Københavns Universitet.

Thurén, Britt-Marie. (2003). Genusforskning - frågor, villkor och utmaningar. Stockholm: Vetenskapsrådet.

Trondman, Mats. (1995). "Vem talar för framtidens förlorare? Om det svenska småstadssamhällets unga arbetarklassmän." I Om unga män. Identitet, kultur och livsvillkor., G. Bohlin og K. Lövgren, 167-92. Lund: Studentlitteratur.

Trondman, Mats, og Nihad Bunar. (2001). Varken ung eller vuxen: "samhället idag är ju helt rubbat." Stockholm: Atlas.

Valestrand, Halldis, Deatra Walsh, Siri Gerrard, og Marit Aure. (2013). "Gendered mobilities in the North." Norsk Geografisk Tidsskrift - Norwegian Journal of Geography 67 (5): 259-259. doi:10.1080/00291951.2013.847856.

Wacquant, Loic J. D. (1996). "The Rise of Advanced Marginality: Notes on Its Nature and Implications." Acta Sociologica 39 (2): 121-39. doi:10.1177/000169939603900201.

Walsh, Deatra, Halldis Valestrand, Siri Gerrard, og Marit Aure. (2013). "Gendered mobilities in the North: Advancing an international comparative perspective." Norsk Geografisk Tidsskrift - Norwegian Journal of Geography 67 (5): 260-65. doi:10.1080/00291951.2013.847857.

Werner, Inga Britt, og Kerstin Annadotter. (2013). "Varför flyttar unga kvinnor fr \a an Gällivare?: Och vad kan samhällsplanering göra för att locka dem tillbaka? Delprojekt Gällivare." Rapport, Kungliga Tekniska Högskolan.

Westman, Liisa. (2005). "Understanding Gendered Welfare in the County of Lapland in Finland." I Med periferien i sentrum: - en studie av lokal velferd, arbeidsmarked og kjønnsrelasjoner i den nordiske periferien, redigeret af Anna-Karin Berglund, Susanne Johansson, og Irene Molina, 113-41. Alta: Norut NIBR Finnmark. 
Weyhe, Torben. (2011). "Finanskrisen og arbejdsmarkedet i Grønland." I Nordiske mænd til omsorgsarbejde!: - en forskningsbaseret erfaringsomsamling på initiativer til at rekruttere, uddanne og fastholde mænd efter finanskrisen, redigeret af Steen Baagøe Nielsen, 248-59. Roskilde: VELPRO - Center for Velfærd, Profession og Hverdagsliv.

Wiborg, Agnete. (2003). "Mobilitet som kulturell kapital og symbol." I Ung i Utkant Aktuel forskning glesbygdsungdomar i Norden., redigeret af Helena Helvé, 340-66. København: Nordisk Ministerråd.

Wilbur, Zelinsky, Janice Monk, og Susan Hanson. (1982). "Women and Geography: A Review and Prospectus." Progress in Human Geography 6 (3): 317-66.

Wildy, Helen, Sigríður Margrét Sigurðardóttir, og Robert Faulkner. (2014). "Leading the Small Rural School in Iceland and Australia Building Leadership Capacity." Educational Management Administration \& Leadership, 1741143213513188. doi:10.1177/1741143213513188.

Wimark, Thomas. (2014). Beyond Bright City Lights: The Migration Patterns of Gay Men and Lesbians. Stockholm: Department of Human Geography, Stockholm University. http://www.diva-portal.org/smash/record.jsf?pid=diva2:713583.

Waara, Peter. (1996). Ungdom i Gränsland. Umeå: Boréa bokförlag.

Waara, Peter. (2002). "At the End of the World - Young People in the Barents Area." Young 10 (3-4): 2-11. doi:10.1177/110330880201000301.

Waara, Peter. (2003). "Sverige." I Ung i Utkant - Aktuel forskning glesbygdsungdomar i Norden., redigeret af Helena Helvé, 187-223. København: Nordisk Ministerråd.

Yndigegn, C. (2003). "Life Planning in the Periphery: Life Chances and Life Perspectives for Young People in the Danish-German Border Region." Young 11 (3): 23551. doi:10.1177/11033088030113003.

Yndigegn, Carsten. (2003). Unge og regional identitet. Forventninger og indstilling til livsbetingelser og livsmuligheder I den dansk-tyske grænseregion. Aabenraa: Institut for grænseforskning.

Zelinsky, Wilbur, Janice Monk, og Susan Hanson. (1982). "Women and Geography: A Review and Prospectus." Progress in Human Geography 6 (3): 317-66.

Ziehe, Thomas. (1996). Ny ungdom og usædvanlige læreprocesser. Kulturel frisættelse og subjektivitet. Copenhagen: Politisk Revy.

Ålands statistik och utredningsbyrå. (2008). Kvinnors foretagande på Åland: En samhällsekonomisk resurs esurs med tillväxtpo. Mariehamn.

Ålands statistik och utredningsbyrå. (2012). Skillnader i kvinnors och mans ekonomiska situation: Publiceras som bilaga till landskapsbudgeten 2012.

Ålands statistik och utredningsbyrå. (2013). Kvinnors och mans val och villkor i samhalle och arbetsliv: Publiceras som bilaga till landskapsbudgeten 2013.

Ålands statistik och utredningsbyrå. (2014). Kvinnors och mäns livsvillkor och hälsa: Publiceras som bilaga till landskapsbudgeten 2014.

Ålands statistik och utredningsbyrå. (2015). Kvinnor och män i livets olika skeden: Könsskillnader med rötter i barn- och ungdomsåren. 


\section{Sammenfatning}

Denne rapport præsenterer en tværnordisk kortlægning af eksisterende forskning og faglitteratur om køn, uddannelse og befolkningsstrømme i yderområder i henholdsvis Norge, Danmark, Sverige, Finland, Island og de selvstyrende områder Grønland, Færøerne samt Åland. Kortlægningen er lavet på opdrag af Nordisk Ministerråd og det danske Ministerium for Børn, Ligestilling, Integration og Sociale Forhold.

Formålet med kortlægningen har været at belyse udviklingstendenser og identificere best practice cases på tværs af Nordens yderområder, og dermed bidrage til erfaringsudveksling og skabe grundlag for fælles drøftelser og diskussion. Et helt central ærinde med denne kortlægning har været at belyse disse udfordringer og samtidig sætte fokus på den tætte sammenhæng, der er mellem køn og sted/lokalitet. Som det fremgår af kortlægningen, er de steder, mænd og kvinder bor, og de sfærer, som de færdes i ( $\mathrm{fx}$ i familien, på uddannelsesinstitutionerne, på arbejdspladsen, osv.) alle forankret i specifikke geografiske lokaliteter, der er med til at definere både hvilke mulighedsstrukturer der eksisterer, og hvilke grader af frihed det enkelte individ oplever at have. Kortlægningen tager således afsæt i en forståelse af, at køn må medtænkes, hvis man til fulde skal forstå udviklingstendenserne i Nordens yderområder.

\section{Kønnede udviklingstendenser i Nordens yderområder}

Kortlægningen fokuser på en række bestemte udviklingstendenser, som har en særlig betydning for yderområdernes levedygtighed og sammenhængskraft, og for de mennesker som bor her. Dette gælder fx udfordringer vedrørende ændrede levevilkår, stagnerende eller negativ økonomisk udvikling og lav vækst, nedgang i antallet af arbejdspladser (især i de traditionelle mandefag) samt ikke mindst migration og affolkning. Det faktum, at kvinderne i højere grad end mændene flytter fra Nordens yderområder og ind mod byerne har givet anledning til begreber som f.eks. kvindeflugt og kvindeunderskud.

Som det fremgår af kortlægningen er det på sin vis ikke nogen ny problemstilling, at kvinderne i de tyndere befolkede områder i Norden flytter ind mod byerne; rent faktisk har forskning i over 100 år peget på 
denne problemstilling, omend de globale udviklingstendenser i nyere tid synes at have bidraget til, at de negative tendenser forstærkes. Samtidig er der indenfor de senere år kommet et stigende fokus på de tilbageblevne mænd, og det fremføres, at omstruktureringerne på arbejdsmarkederne i yderområderne (og en markant nedgang i antallet af de såkaldte "mandearbejdspladser") har sat de mere traditionelle former for maskulinitet under pres. I denne debat er mændene i yderområderne blevet beskrevet som omstillingsmodvillige, uuddannede og som tabere, der er ude af trit med samfundsudviklingen. Vi har altså igennem de senere år set et skifte, hvor det ikke bare bliver italesat som et problem, at kvinderne i Nordens yderområder vælger at flytte, dvs. at de forsvinder fra områderne, men nu også italesættes som et problem, at mændene i disse områder ikke vælger at flytte. Det er med udgangspunkt i denne problemstilling, at vi i denne rapport har arbejdet på at afdække, hvilken sammenhæng der er mellem sted/lokalitet og køn, og herunder kortlægge den litteratur der findes på området.

I kortlægningen inddrages frem for alt udfordringer, som har med uddannelse/efteruddannelse, arbejde og fritid at gøre. Som det fremgår, rummer de beskrevne udfordringer mange dimensioner - både på individ-, gruppe- og samfundsniveau, og i samspillet niveauerne imellem.

Samlet set er der en række relevante lighedstræk på tværs af disse forskellige nordiske lokaliteter. Dette kommer til udtryk i uddannelsessystemet, på arbejdsmarkedet og generelt i lokalsamfundene i de nordiske yderområder. Når man betragter de nordiske lande samlet er der imidlertid også en række kontrasterende forskelligheder, som bidrager til at gøre de udfordringer, som kortlægningen fokuserer på, endnu mere komplekse. Forskellighederne angår eksempelvis geografiske distancer, befolkningstæthed, fordelingen af naturressourcer, organisering af den sociale infrastruktur, hastigheden hvormed de nationale, regionale og lokale uddannelses- og arbejdsmarkeder ændrer sig, forskelle i karakteristikken af disse arbejdsmarkeder, og hvilke sektorer der dominerer.

På Island, Færøerne og i Grønland samt Åland gælder det, at der er tale om mindre øsamfund med mærkbart færre indbyggere end i det øvrige Norden. Det har betydning for karakteren af forandringerne, samt for diskussionerne om hvordan de kan imødegås. Også på disse steder gælder det dog, at udviklingstendenserne rummer en tydelig kønsdimension. 


\section{Udvalgte temaer og tendenser på tværs af Norden}

I dette kapitel, som udgør rapportens sammenfatning, er det vigtigt at understrege, at et sådant format ikke levner særlig megen plads til forskelle, variationer og nuancer; hverken i forhold til at berøre forskelle og variationer i de forskellige nordiske lande eller i forhold til at medtage variationer og nuancer, hvad angår køn. Det er desværre en dimension, som det er svært at komme udenom, når man sammenfatter og opsummerer, især når emnet har været så bredt og omfattende, som det er tilfældet i denne kortlægning. Vi vil derfor gerne understrege, at de enkelte kapitler i kortlægningen bør læses, hvis man som læser ønsker en indgående forståelse af hvilken rolle køn, uddannelse og befolkningsstrømme spiller i Nordens yderområder.

Med ovennævnte forbehold prøver vi ikke desto mindre i det følgende at opsummeres en række gennemgående temaer og tendenser, som kortlægningen har identificeret:

\section{Der er nationale variationer i typen og mængden af litteratur på området}

Kortlægningen har haft som ærinde at identificere forskning og faglitteratur om køn, uddannelse og befolkningsstrømme i Norden. Vores desk research viser, at der er nationale variationer i typen og mængden af litteratur, fx er der tydeligvis i Norge et rigt felt af forskere, der beskæftiger sig med uddannelse, befolkningsstrømme og yderområdeproblematikker - og i nogen grad også med en kønsvinkel - mens det i andre af de nordiske lande har været vanskeligere at identificere litteratur på området.

\section{Der mangler forskning og faglitteratur med et kønsperspektiv}

På baggrund af den identificerede litteratur kan vi konstatere, at køn som kategori er fraværende i en stor del af litteraturen på området. Det er litteratur, som bl.a. er skrevet af uddannelsesforskere, landdistriktsforskere, osv. og heri præsenteres der som udgangspunkt analyser af unge og uddannelse generelt, eller af yderområdeproblematikker generelt, og ofte uden at forskelle og/eller ligheder mellem henholdsvis drenge/unge mænd og piger/unge kvinder analyseres. Der er altså tale om, at kønsanalyser i en stor del af litteraturen på feltet er ikkeeksisterende, eller i hvert fald ofte er nedtonet, mens andre kategorier (som fx etnicitet eller social klasse eller endnu helt andre kategorier som fx "uddannelsesparathed" eller "udsathed") vies større opmærksomhed. Denne tendens gælder ikke kun indenfor forskningen, men gør sig også gældende, når køn, uddannelse og befolkningsstrømme diskuteres 
blandt politiske aktører eller indenfor praksis (fx på uddannelsesinstitutionerne). Også her diskuteres køn sjældent i nævneværdigt omfang; ofte fordi køn opfattes som irrelevant eller fordi køn forstås ud fra et biologisk perspektiv, dvs. betragtes som "naturlige" iboende egenskaber ved individuelle unge, som det derfor ikke vurderes muligt at ændre på.

\section{Udviklingstendenserne i Nordens yderområder bør/kan ikke analyseres og ej heller løses uden af medtænke kønsperspektivet}

Køn er imidlertid ikke bare noget man "er" eller "har", men også noget som "gøres." Køn handler m.a.o. også om sociale handlinger, om forestillinger og forventninger samt om mulighedsstrukturer, og derfor rummer udviklingstendenserne i Nordens yderområder - som kortlægningen også illustrerer - dimensioner, som ikke bør/kan analyseres og ej heller løses uden at medtænke kønsperspektivet. Det drejer sig bl.a. om ændrede lokale erhvervstraditioner og uddannelsesmønstre i yderområderne, udfordringer vedr. transport, social infrastruktur, lokale tilknytningsmønstre eller mangel på samme, arbejdsmarkedernes kønsopdeling, ændring af rekrutteringsmønstre, osv. Som et resultat af processer knyttet til strukturelle, økonomiske og socio-kulturelle ændringer viser litteraturen, at yderområderne står overfor en del udfordringer. Mens nogle tilpasser sig de nye vilkår, hægtes andre af, og som kortlægningen illustrerer synes, især mændene at blive ramt af udviklingen i yderområderne.

\section{De unge i yderområderne føler et stort pres for at forlade deres lokalsamfund}

Litteraturen viser, at de unge afhængigt af hvor de bor, træffer deres valg på baggrund af meget forskellige vilkår, fordi de unge i Nordens yderområder ofte enten er decideret tvunget til og/eller føler et stort pres for at forlade deres lokalsamfund. Man kan med andre ord tale om, at ungdommen i de nordiske yderområder mærker de ændrede mønstre for uddannelse og erhverv samt stigende krav om mobilitet særligt tydeligt. I modsætning til de unge, der bor centralt, føler de unge - og især kvinderne - et pres for at forlade deres lokalsamfund. I visse yderområder har de unge slet ikke adgang til hverken en ungdomsuddannelse eller videregående uddannelse, eller kun adgang til et begrænset udvalg af uddannelser og/eller studieretninger, hvilket i praksis kan betyde, at de er tvunget til at flytte hjemmefra i en ganske tidlig alder. Tidligere studier viser, at det især er de unge kvinder i yderområderne, der flytter tidligt hjemmefra. Dog skal det her siges, at der er forskellige traditioner på tværs af de Nordiske lande; fx er der i både Grønland, Island og på Færøerne tradition for at unge forlader stederne og rejser væk (primært til Danmark) - for at tage en uddannelse. Men hvor en stor del af grøn- 
landske og færøske unge ikke vender hjem igen, er der i Island større tradition for, at de unge vender hjem igen og bruger deres erhvervede uddannelse her. Den afdækkede litteratur understreger altså, at unge af begge køn forlader deres hjemstavn i yderområderne, ikke kun fordi de $\emptyset n s k e r$ at se og opleve verden eller fordi dét at flytte er et naturligt led i at blive voksen, men også fordi det føles umuligt for dem at blive enten pga. manglende uddannelses- og jobmuligheder, eller fordi de finder lokalsamfundene i yderområderne begrænsende eller klaustrofobiske.

\section{Litteraturen viser, at det ofte er uddannelses- eller arbejdsrelateret, når unge (især kvinderne) flytter fra yderområderne}

Kortlægningen viser endvidere, at de unge i Nordens yderområder har forskellige forhåbninger om, hvorvidt der på længere sigt er en plads til dem det sted, de bor. Og netop her viser litteraturen, at køn synes at spille en væsentlig rolle, idet der på tværs af den nordiske litteratur peges på, at de ændrede vilkår i yderområderne gør, at de unge kvinder her i højere grad oplever, at der er begrænsninger knyttet til deres køn. Dette synes i højere grad, end det gør sig gældende for de unge mænd, at kollidere med de unge kvinders ønsker om, hvordan de gerne vil leve på sigt, og hvad der faktisk er muligt de steder, de bor. Således fremgår det af kortlægningen, at de unge kvinder i yderområderne synes at satse på videregående uddannelse i højere grad end de unge mænd. Litteraturen på området peger endvidere på, at kvindernes ønske om at flytte fra yderområderne i nogen grad kan knyttes sammen med et ønske fra kvindernes side om at få adgang til flere og lige muligheder - indenfor uddannelse, arbejdsliv og i hverdagslivet - samt en større grad af frihed fra kønstraditionelle forventninger og normer.

\section{Stedet, hvor de unge bor, danner præmisserne for deres muligheder og valg i forhold til uddannelse}

Selvom vi i Norden på nationalt plan har et politisk ideal om ligestilling, er der, som kortlægningen viser, kontekstspecifikke variationer. På den måde kan man tale om, at den norm om lige muligheder for alle, som kommunikeres i de nordiske uddannelsessystemer ikke altid korresponderer med den faktiske situation i Nordens yderområder. Kortlægningen peger således på, at det på tværs af Norden er vigtigt at stille spørgsmålet om, hvorvidt mænd og kvinder nu også reelt har lige adgang til at opnå den uddannelse/efteruddannelse, de måtte ønske sig. Det sted, de unge bor, sætter sine begrænsninger for, hvad der er realistisk og tilgængeligt, og således er stedet med til at forme den mulighedshorisont, som de unge forholder sig til. 


\section{Stedet, hvor de unge bor, danner tillige præmisserne for engagement og lyst til læring}

At de steder, de unge bor, er med til at danne præmisserne gælder ikke bare for de unges muligheder og valg, hvad angår uddannelse og arbejdsliv, men i nogen grad også for deres engagement og lyst til læring. I nogle yderområder tilsiger det lokale arbejdsmarked og erhvervsliv mindre uddannelse, hvormed det at fravælge uddannelse fremstår som et fornuftigt og rationelt valg, eller uddannelsesinstitutionernes kultur opleves af nogle elever som "fremmed", fordi deres identiteter er forankret i et lokalsamfund, hvor fokus er på andre værdier og former for kulturel/social kapital end dem som er værdsat indenfor uddannelsessystemet. Her tales der indenfor forskningen på området om, at de unge i yderområderne, især de unge mænd, potentielt oplever en kulturel kollision; en kollision mellem på den ene side de værdier og den opfattelse af hvad der kendetegner "det gode liv" de unge mænd har, og på den anden side den uddannelses- og karriereorienterede fortælling (diskurs), som fremføres bl.a. i medierne og af politikere på den nationale scene, og som cementerer, at man "kun er noget, hvis man bliver til noget" - eller at man er mest og når længst, hvis man tager en boglig uddannelse.

\section{Den geografiske mobilitet er ofte koblet til social mobilitet}

Som det fremgår af forskningen på området, er uddannelse af central betydning for unges livsforløb både i form af tilpasninger til det omgivende arbejdsmarked og udformning af levevis, livsstil og identitet. For unge fra de nordiske yderområder og fra de mindre bysamfund i disse områder indebærer det at vælge en videregående uddannelse ofte også flytning fra hjemstedet. Samtidig er den geografiske mobilitet i mange tilfælde tillige koblet til social mobilitet, idet en del af de unge fra yderområderne kommer fra hjem og/eller lokale miljøer, hvor det at tage videregående uddannelse ikke har været sædvane.

\section{De unge kvinder i periferien oplever at have færre muligheder på de lokale arbejdsmarkeder, i det lokale foreningsliv samt ved fritidsaktiviteter og kulturelle udbud}

Litteraturen på området fremhæver på tværs af Norden, at de unge i yderområderne i høj grad er underlagt en række strukturelle vilkår ikke bare i forbindelse med deres valg af uddannelse, men også hvad angår erhverv og arbejdsliv (adgang til arbejdsmarkedet, geografiske afstande, infrastruktur, mulighed for praktikpladser, osv.). Når de unge kvinder i periferien ofte oplever at have færre muligheder end de unge mænd, handler det bl.a. om, at jobmulighederne i yderområderne (ofte indenfor den primære sektor) ofte ikke er tilgængelige for dem i samme omfang. 
Men der er også andre aspekter, der fremhæves i forskningen på området; bl.a. at typen og graden af foreningsliv, fritidsaktiviteter og kulturelle udbud spiller en vigtig rolle i de unges valg om enten at blive i yderområderne eller at flytte - og her peger litteraturen på, at de aktiviteter, som de unge mænd deltager i, synes at knytte dem mere til stedet, end de aktiviteter, som de unge kvinder deltager i.

Kønsrelationer og kønsnormer er en vigtig del af ungdommens syn på det lokale arbejdsmarked og på deres følelse af stedtilknytning

Det lokale (og regionale) arbejdsmarked i yderområderne danner en ramme, som de unge vurderer deres muligheder op i mod. Litteraturen på området peger på, at det ikke kun er uddannelsesmulighederne, men også mulighederne på arbejdsmarkedet, der har betydning for ungdommens, især kvindernes, ønske om at ville flytte væk eller på sigt vende hjem igen. Både det kønsopdelte uddannelsesvalg og det kønsopdelte arbejdsmarked er udtalt i Nordens yderområder. Noget tyder på, at flere og flere unge kvinder bryder med de traditionelle grænser/forventninger, og dermed statuerer eksempler som andre kan lade sig inspirere af, men spørgsmålet er, om de unge mænd i yderområderne i samme grad opmuntres til at vælge utraditionelt? Litteraturen på området peger under alle omstændigheder på, at der er en tendens til, at de unge mænd har en større grad af stedtilknytning end de unge kvinder. Dette kan skyldes, at de unge mænd ofte er tættere forbundet med de typer af erhverv og livsmønstre, som samtidig har historisk og geografisk forankring i yderområdernes lokaliteter.

\section{Det er vigtigt at kunne udbyde mere varierede muligheder og arbejdsformer i yderområderne}

Når forskningen på området viser, at det for ungdommen, især for kvinderne, særligt er uddannelses- og arbejdsmulighederne, der forklarer, hvorfor de ønsker at flytte væk, peger det på vigtigheden af at arbejde på at kunne udbyde mere varierede muligheder og arbejdsformer i yderområderne. Med henblik på at kunne sikre at flere af de unge kvinder vælger at blive eller vælger at flytte tilbage til deres lokalområder efter endt uddannelse, synes det helt konkret at være nødvendigt at skabe lokale arbejdsmuligheder i yderområderne, som forudsætter videregående uddannelse. 


\section{Den stærke kønssegregering af de nordiske arbejdsmarkeder forstærker de negative udviklingstendenser i yderområderne}

Et fællestræk på tværs af de nordiske landes arbejdsmarkeder er en stærk tendens til kønssegregering - mere udtalt i visse lande fremfor andre, men ikke desto mindre er der en generel tendens til, at kvinderne arbejder i den offentlige sektor og mændene i den private - og at særligt den primære sektor (landbrug, skovbrug, råstofudvinding) i høj grad er domineret af mænd. Denne kønssegregering af arbejdsmarkedet har i flere nordiske yderområder været medvirkende årsag til, at særligt mændene har været hårdt ramt af den økonomiske krise, idet antallet af traditionelle mandejobs er faldet betydeligt i flere områder. Kortlægningen af litteraturen vedr. udviklingstendenser på arbejdsmarkederne i yderområderne understreger, at en vigtig nøgle til at skabe forandringer i uddannelsesmønstrene og befolkningsstrømningerne, kan ligge i at bløde op for forestillinger om "mandejobs" og "kvindejobs". Hvis de lokale arbejdsmarkeder kan afmontere disse skel, ville det dels udvide udbuddet af oplevede job- og uddannelsesmuligheder for såvel unge mænd som kvinder, og dels medvirke til at skabe det opgør med traditionelle kønsnormer, som de unge kvinder ellers opsøger via deres flytning til større lokaliteter.

\section{Yderområderne rummer potentielt iboende patriarkalske strukturer, der skubber de unge kvinder væk}

I en del af litteraturen er det blevet fremhævet, at yderområderne har en tendens til at skubbe kvinderne væk bl.a. pga. deres iboende patriarkalske strukturer. Således bliver det bl.a. i en del af litteraturen konkluderet, at flere af de unge kvinder betragter dét at flytte bort fra de små lokalsamfund i yderområderne og tage en uddannelse som afgørende for at kunne udforme egen identitet og skabe en livsstil frikoblet fra lokale kulturelle og strukturelle begrænsninger; herunder kønnede forventninger til kvinders rolle/ansvar, parforhold og moderskab - kønnede forventninger som disse unge kvinder oplever vil begrænse deres handlingsrum. Vores desk research peger på, at der mangler forskning om, hvilke uskrevne regler og normer, der regulerer relationerne mellem mænd og kvinder i yderområderne. 


\section{I yderområderne bor også mænd, som har truffet bevidste valg - som bliver boende, fordi de trives netop her}

I diskussionen om det kvindeunderskud, der præger mange af Nordens yderområder, er det også relevant at understrege, at det ikke kun handler om at lokke nye tilflyttere (især kvinder) til yderområderne, men at det også kan handle om at værne om dem (især mænd), som bliver boende og i forlængelse herfra at løfte mere positive billeder frem. Tendensen til at de unge mænd har en større grad af stedtilknytning end de unge kvinder handler nemlig ikke kun om en særlig type livsmønster, om faglige fællesskaber i relation til lokale erhvervstraditioner eller om at fritidstilbuddene i yderområderne harmonerer godt med traditionelle mandeinteresser - det handler ifølge litteraturen også om, at de unge mænd bliver boende, fordi de foretrækker livet på landet, og fordi de oplever, at der er nogle værdier, som de kun/bedst kan praktisere her. At de tilbageblevne mænd i yderområderne kan hænde at have truffet bevidste og velovervejede valg om bopæl, uddannelse og erhverv, indgår i mindre grad som et element i den tværnordiske debat.

\section{Nogle mænd i yderområderne bliver særligt ramt af de lokale arbejdsmarkeders restrukturering og de ændrede levevilkår i yderområderne}

Af litteraturen på området fremgår det, at mændene synes at blive særligt ramt af den restrukturering af arbejdsmarkedet og de ændrede livsvilkår, som kendetegner yderområderne i alle de nordiske lande. Det er ydermere blevet fremhævet, at de senere års finanskrise har skubbet denne udvikling yderligere på vej, hvorfor man i et ligestillingsperspektiv ikke taler om the recession, men om the mancession. Dette skal sammenholdes med, at der i dag er stor fokus på mobilitet og fleksibilitet, hvilket ifølge forskningslitteraturen er med til at skabe en særlig negativ fortælling om de tilbageblevne mænd i yderområderne. Litteraturen peger på, at der er mænd i yderområderne, som oplever ambivalens, og nogle udviser også tegn på det, der inden for forskningslitteraturen, er blevet omtalt som melankolsk maskulinitet. Dette skal ses i sammenhæng med, at mange af de unge mænd i yderområderne er vokset op med en traditionel kønsforståelse, hvor maskulinitet og hårdt fysisk arbejde er tæt forbundet. Med omstruktureringen på de lokale arbejdsmarkeder, og stigende arbejdsløshed indenfor de traditionelle mandefag, bliver denne forståelse udfordret. 


\section{Yderområdernes negative stigma påvirker de unge af begge køn}

Af kortlægningen fremgår det endvidere, at der findes forskellige negative forestillinger om og italesættelser af de nordiske yderområder, og de mennesker som bor her. Dette negative stigma er ikke kun forankret i medierne; det høres også jævnligt blandt politikere, praktikere, forskere og ikke mindst registreres det hos de mennesker, især de unge, der bor i yderområderne. Indenfor forskningen tales der her om såkaldte overlokale strømninger; herunder bl.a. billeder af hvad der kendetegner "det gode liv" og fortællinger knyttet til yderområderne, og som fremføres i både trykte, digitale og sociale medier og på nettet i øvrigt. Netop internettet og de sociale medier er vigtige arenaer i de unges daglige liv, og de udgør for mange unge også i yderområderne en vigtig referenceramme for den måde, hvorpå de ser sig selv, og de tanker de gør sig om fremtiden.

\section{Der må nye fortællinger til: Yderområderne som steder, der skal næres og bevares?}

Ifølge den afdækkede litteratur spiller yderområdernes negative stigma en ganske central rolle i forhold til de udviklingstendenser, som kortlægningen fokuserer på - dog peger kortlægningen på, at der mangler yderligere viden om denne dimension, og eventuelle kønsforskelle knyttet hertil. Noget tyder på, at unge af begge køn i yderområderne har en negativ vurdering af deres egen hjemstavn, og ungdommen her, og især de unge mænd, oplever tilsyneladende, at det forventes, at de flytter for ikke at være/fremstå som tilbagestående (som umoderne). Den negative fortælling om de nordiske yderområder indikerer, at de opfattes som steder, der må forandres (moderniseres), snarere end steder, der skal næres og bevares. Her peger forskning på, at den opfattelse, som de unge får af yderområderne er vigtigere end de faktiske forhold. Her er det i litteraturen blevet påpeget, at man ved at fremme andre billeder og muligheder kan medvirke til, at nye mønstre og strategier udvikles. 
Ved Stranden 18

DK-1061 Copenhagen $\mathrm{K}$

www.norden.org

\section{Place, (In)Equality and Gender}

This mapping presents a selected overview of existing research on gender, education and population flows in the Nordic peripheral areas. These areas are faced with a series of challenges that cannot be analyzed nor solved without taking a gender perspective into account. The challenges relate to, for instance, altered living conditions caused by global changes, stagnated or negative economic development, decrease in the amount of workplaces (particularly in the traditionally male-dominated professions) as well as, not least, migration and depopulation which is partly due to the fact that the young people of the area (especially the women) move to bigger cities to educate themselves. The challenges in question are not only significant in relation to the viability and cohesion of the areas, but also for the men and women who live there and their mutual social relations.

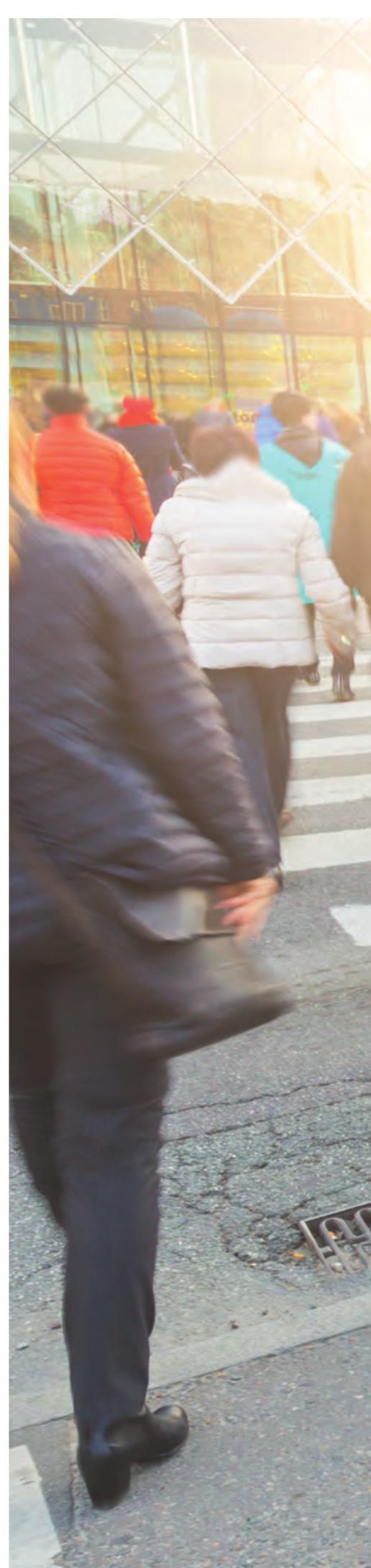

